Prepared in cooperation with the Uintah Water Conservancy District and the Bureau of Reclamation

\title{
Groundwater and Surface-Water Resources near Red Fleet Reservoir, Uintah County, Utah
}

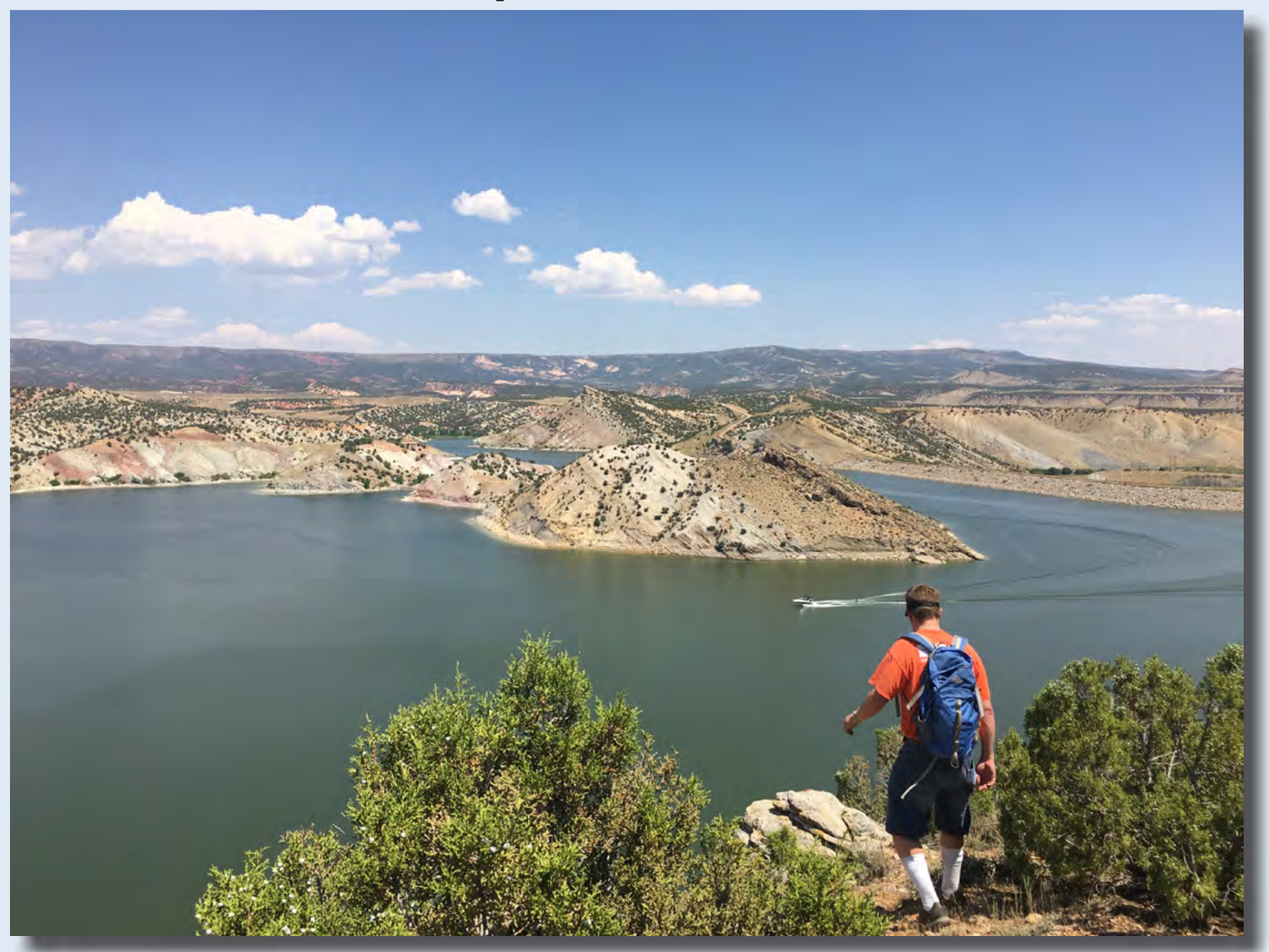

Scientific Investigations Report 2019-5101 
Cover: View of Red Fleet Reservoir looking northeast near well DH-109; photograph taken by Mike Hess, U.S. Geological Survey, August 2016. 


\section{Groundwater and Surface-Water Resources near Red Fleet Reservoir, Uintah County, Utah}

By Thomas M. Marston, John E. Solder, and Katherine K. Jones

Prepared in cooperation with the Uintah Water Conservancy District and the

Bureau of Reclamation

Scientific Investigations Report 2019-5101 


\title{
U.S. Department of the Interior DAVID BERNHARDT, Secretary
}

\author{
U.S. Geological Survey \\ James F. Reilly II, Director
}

U.S. Geological Survey, Reston, Virginia: 2019

For more information on the USGS - the Federal source for science about the Earth, its natural and living resources, natural hazards, and the environment-visit https://www.usgs.gov or call 1-888-ASK-USGS.

For an overview of USGS information products, including maps, imagery, and publications, visit https://store.usgs.gov.

Any use of trade, firm, or product names is for descriptive purposes only and does not imply endorsement by the U.S. Government.

Although this information product, for the most part, is in the public domain, it also may contain copyrighted materials as noted in the text. Permission to reproduce copyrighted items must be secured from the copyright owner.

Suggested citation:

Marston, T.M., Solder, J.E., and Jones, K.K., 2019, Groundwater and surface-water resources near Red Fleet Reservoir, Uintah County, Utah: U.S. Geological Survey Scientific Investigations Report 2019-5101, 40 p., https://doi.org/10.3133/sir20195101. 


\section{Contents}

Abstract

Introduction

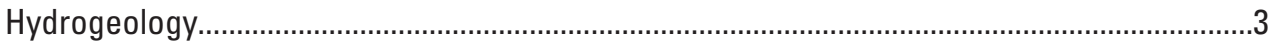

Assessment of Groundwater Interaction with Red Fleet Reservoir................................................

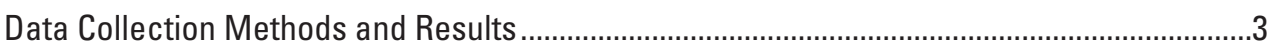

Surface-Water Inflow to and Outflow from Red Fleet Reservoir.......................................3

Pumped Outflow from Red Fleet Reservoir ..................................................................5

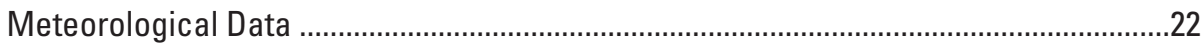

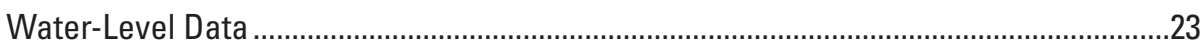

Groundwater Movement ..........................................................................................24

Estimates of Groundwater Recharge and Discharge from Red Fleet Reservoir......................25

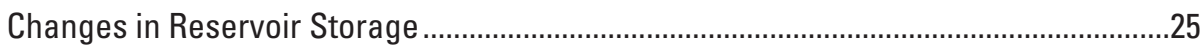

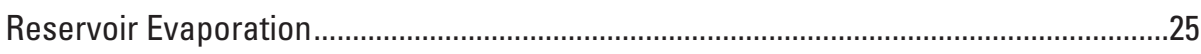

Estimates of Groundwater Recharge and Discharge at Red Fleet Reservoir .................26

Big Brush Creek Seepage Assessment Upstream of Red Fleet Reservoir .....................27

Evaluation of Aquifer Properties in the Nugget Sandstone Aquifer near Red Fleet Reservoir ......28

Aquifer Test Description and Analysis ...................................................................................28

Evaluation of Groundwater Geochemical Characteristics in the Nugget and

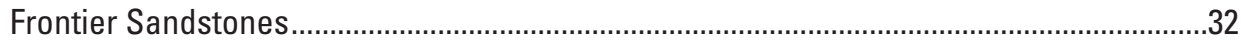

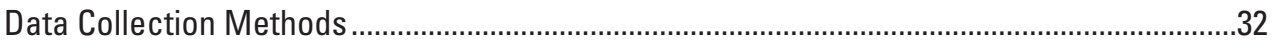

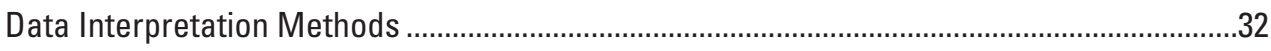

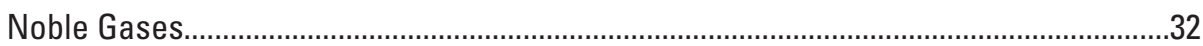

Tritium and Helium Isotopes.....................................................................................3

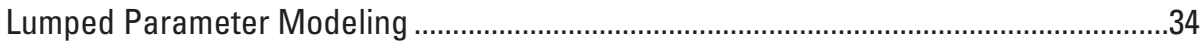

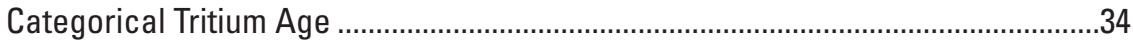

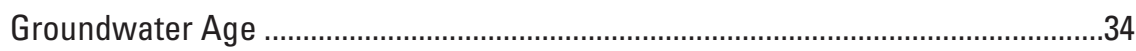

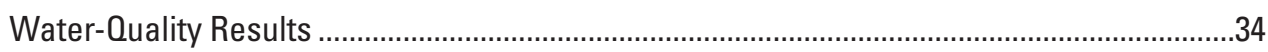

Major lons, Nutrients, and Trace Metals .....................................................................34

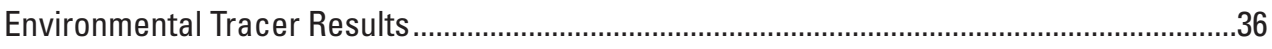

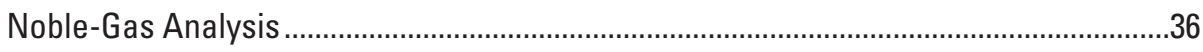

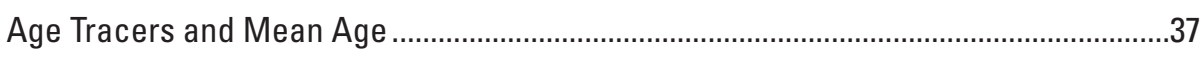

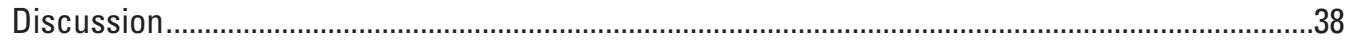

Summary.

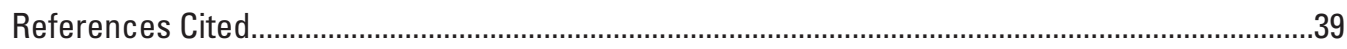




\section{Figures}

1. Image showing location of the Red Fleet Reservoir study area, Uintah County, Utah .....2

2. Map showing gocation of wells, streamgage, and pump station near Red Fleet Reservoir, Utah

3. Graph showing annual inflows and outflows to and from Red Fleet Reservoir, near Vernal, Utah, 1980-2015.

4. Graph showing annual precipitation at Vernal Airport and Natural Resources Conservation Service Split Mountain weather stations, Utah, 1980-2015.

5. Graph showing water-level altitude in selected wells and Red Fleet Reservoir stage, Uintah County, Utah, 2016-17.

6. Image showing potentiometric contours of the Nugget and Frontier Sandstone aquifers in September 2017, Red Fleet Reservoir, Utah ..

7. Graph showing reservoir stage and calculated groundwater recharge/discharge at Red Fleet Reservoir, Utah, 1980-2015

8. Image showing locations of seepage measurements taken on November 17, 2017, on Big Brush Creek upstream of Red Fleet Reservoir, Utah.

9. Graph showing measured discharge and uncertainty at two stations on Big Brush Creek upstream of Red Fleet Reservoir, Utah

10. Graph showing recorded discharge at the U.S. Geological Survey Big Brush Creek streamgage 09261700 during the November 17, 2017, seepage assessment above Red Fleet Reservoir, Utah.

11. Image showing locations of wells and discharge pipe used during the March 2017 Nugget Sandstone aquifer test at Red Fleet Reservoir, Utah.

12. Graph showing measured change in water level from observation well RF1 near Red Fleet Reservoir resulting from pumping at the Red Fleet State Park well, and simulated change in water level from a Theis aquifer-response solution, March 2017, Red Fleet Reservoir, Utah.....

13. Trilinear diagram showing relative major-ion concentrations for selected wells near Red Fleet Reservoir, Utah.

14. Graph showing modeled noble-gas recharge temperature and elevation for groundwater sampled near Red Fleet Reservoir, Utah

15. Graph showing age-tracer cross-plot of groundwater near Red Fleet Reservoir, Utah.

\section{Tables}

1. Reservoir data, evaporation, and calculated recharge and discharge from Red Fleet Reservoir, Utah, 1980-2015.

2. Field measurements and major ions, selected trace elements, and nutrient concentrations in groundwater and surface water from selected sites near Red Fleet Reservoir, Utah

3. Noble-gas model results from samples collected at Red Fleet Reservoir, Utah

4. Age-tracer concentrations and estimated mean age and age distribution of groundwater near Red Fleet Reservoir, Utah. 


\section{Conversion Factors}

U.S. customary units to International System of Units

\begin{tabular}{|c|c|c|}
\hline Multiply & By & To obtain \\
\hline \multicolumn{3}{|c|}{ Length } \\
\hline inch (in.) & 2.54 & centimeter $(\mathrm{cm})$ \\
\hline foot $(\mathrm{ft})$ & 0.3048 & $\operatorname{meter}(\mathrm{m})$ \\
\hline mile (mi) & 1.609 & kilometer $(\mathrm{km})$ \\
\hline \multicolumn{3}{|c|}{ Area } \\
\hline acre & 4,047 & square meter $\left(\mathrm{m}^{2}\right)$ \\
\hline acre & 0.004047 & square kilometer $\left(\mathrm{km}^{2}\right)$ \\
\hline square foot $\left(\mathrm{ft}^{2}\right)$ & 929.0 & square centimeter $\left(\mathrm{cm}^{2}\right)$ \\
\hline square inch $\left(\right.$ in $\left.^{2}\right)$ & 6.452 & square centimeter $\left(\mathrm{cm}^{2}\right)$ \\
\hline \multicolumn{3}{|c|}{ Volume } \\
\hline gallon (gal) & 3.785 & liter (L) \\
\hline cubic foot $\left(\mathrm{ft}^{3}\right)$ & 0.02832 & cubic meter $\left(\mathrm{m}^{3}\right)$ \\
\hline acre-foot (acre-ft) & 1,233 & cubic meter $\left(\mathrm{m}^{3}\right)$ \\
\hline \multicolumn{3}{|c|}{ Flow rate } \\
\hline gallon per minute (gal $/ \mathrm{min})$ & 0.06309 & liter per second $(\mathrm{L} / \mathrm{s})$ \\
\hline \multicolumn{3}{|c|}{ Hydraulic conductivity } \\
\hline foot per day $(\mathrm{ft} / \mathrm{d})$ & 0.3048 & meter per day $(\mathrm{m} / \mathrm{d})$ \\
\hline
\end{tabular}

Temperature in degrees Celsius $\left({ }^{\circ} \mathrm{C}\right)$ may be converted to degrees Fahrenheit $\left({ }^{\circ} \mathrm{F}\right)$ as follows:

$$
{ }^{\circ} \mathrm{F}=\left(1.8 \times{ }^{\circ} \mathrm{C}\right)+32 \text {. }
$$

\section{Datums}

Vertical coordinate information is referenced to the North American Vertical Datum of 1988 (NAVD 88).

Horizontal coordinate information is referenced to the North American Datum of 1983 (NAD 83). Altitude, as used in this report, refers to distance above the vertical datum.

\section{Supplemental Information}

Specific conductance is given in microsiemens per centimeter at 25 degrees Celsius $(\mu \mathrm{S} / \mathrm{cm}$ at $\left.25^{\circ} \mathrm{C}\right)$.

Total dissolved gas pressure is reported in millimeters of mercury $(\mathrm{mm} \mathrm{Hg})$, where $760 \mathrm{~mm} \mathrm{Hg}$ equals one atmosphere.

Concentrations of chemical constituents in water are given in either milligrams per liter (mg/L) or micrograms per liter $(\mu \mathrm{g} / \mathrm{L})$.

Stable isotope concentration is reported as per mil, which is equivalent to parts per thousand.

Tritium units (TU) are used to report tritium concentration, where one TU equals tritium concentration in picoCuries per liter divided by 3.22 . 
Chlorofluorocarbon concentrations are reported as picomoles per kilogram $(\mathrm{pmol} / \mathrm{kg})$.

Sulfur hexafluoride concentrations are reported as femtomoles per kilogram (fmol/ $/ \mathrm{kg}$ ).

\section{Abbreviations}

\begin{tabular}{|c|c|}
\hline${ }^{3} \mathrm{H}$ & tritium \\
\hline${ }^{3} \mathrm{He}$ & helium-3 \\
\hline${ }^{3} \mathrm{He} e_{\text {trit }}$ & tritiogenic helium-3 \\
\hline${ }^{4} \mathrm{He}$ & helium-4 \\
\hline${ }^{4} \mathrm{He} e_{\text {terr }}$ & terrigenic helium-4 \\
\hline AVWTP & Ashley Valley Water Treatment Plant \\
\hline CE & closed-system equilibration \\
\hline CFC-11 & trichlorofluoromethane \\
\hline CFC-113 & 1,1,2-trichloro-1,2,2-trifluoroethane \\
\hline CFC-12 & dichlorodifluoromethane \\
\hline CFC & chlorofluorocarbon \\
\hline DM & dispersion model \\
\hline EPA & Environmental Protection Agency \\
\hline $\mathrm{He}$ & helium \\
\hline $\mathrm{He}_{\text {terr }}$ & terrigenic helium \\
\hline $\mathrm{Kr}$ & krypton \\
\hline LPM & lumped parameter modeling \\
\hline $\mathrm{ml}$ & milliliter \\
\hline NGT & noble-gas recharge temperature \\
\hline NRCS & Natural Resources Conservation Service \\
\hline PET & potential evaporation \\
\hline RFSP & Red Fleet State Park \\
\hline SCAN & Soil Climate Analysis Network \\
\hline $\mathrm{SF}_{6}$ & sulfur hexafluoride \\
\hline TDG & total dissolved gas \\
\hline BOR & Bureau of Reclamation \\
\hline USGS & U.S. Geological Survey \\
\hline UWCD & Uintah Water Conservancy District \\
\hline $\mathrm{Xe}$ & xenon \\
\hline$\sigma$ & standard deviation \\
\hline$\chi^{2}$ & chi-squared statistic \\
\hline
\end{tabular}




\title{
Groundwater and Surface-Water Resources near Red Fleet Reservoir, Uintah County, Utah
}

\author{
By Thomas M. Marston, John E. Solder, and Katherine K. Jones
}

\section{Abstract}

Red Fleet Reservoir in Uintah County, Utah, is an approximately 26,000 acre-foot (acre-ft) on-channel reservoir in the Big Brush Creek drainage on the south slope of the Uinta Mountains. It is operated primarily for irrigation needs while providing a supplemental drinking-water supply to the Vernal, Utah area. Red Fleet Reservoir, which was operated by the Bureau of Reclamation and the Uintah Water Conservancy District through 2015, began storing water in May 1980.

The reservoir is on southward dipping Mesozoic lithologies ranging from Jurassic to Cretaceous in age. The Nugget and Frontier Sandstone aquifers are the targeted units in this investigation, which is to characterize groundwater conditions that exist in each sandstone aquifer and how they interact with Red Fleet Reservoir. Groundwater levels were measured in six wells and one spring in the Nugget Sandstone and the Frontier Sandstone aquifers. Water levels in the Nugget Sandstone aquifer were 35-70 feet above the maximum stage of Red Fleet Reservoir on the west and east banks. Water levels in the Frontier Sandstone aquifer were 15-30 feet below the observed stage of Red Fleet Reservoir on the west bank during the study period.

A water budget was calculated for Red Fleet Reservoir between May 1980 and December 2015. During this period, 1,050,000 acre-ft of water from Big Brush Creek discharged into the reservoir, while 993,000 acre-ft of water was released downstream of Red Fleet Dam. Total evaporation from May 1980 through December 2015 was about 52,000 acre-ft, while total precipitation over the same period was about 12,000 acre-ft. From May 1980 through December 2015, the total pumped volume of water from the Tyzack Pump Station, at the base of Red Fleet Dam, was about 42,000 acre-ft. Total groundwater discharge to Red Fleet Reservoir from 1980 through 2015 was about 40,000 acre-ft.

Water was sampled from four wells and from the inflow arm of Red Fleet Reservoir, and analyzed for major-ion chemistry, selected trace metals, nutrients, and environmental tracers. Water sampled from the Nugget Sandstone aquifer yielded good-quality water with dissolvedsolids concentrations of less than 200 milligrams per liter, and no trace elements above the Environmental Protection Agency drinking-water standards. Water sampled from the Frontier Sandstone aquifer yielded poor-quality water with dissolved-solids concentrations of about 2,150 milligrams per liter and arsenic approaching the drinking-water standard of 10 milligrams per liter. Dissolved noble gases used to identify recharge elevations and temperatures for groundwater indicate that water in the Nugget Sandstone aquifer likely recharged at a high altitude and low temperature, and not locally because of interaction with Red Fleet Reservoir. The Frontier Sandstone aquifer is likely recharged at low elevation and at temperatures similar to those observed at Red Fleet Reservoir.

\section{Introduction}

Red Fleet Reservoir (fig. 1) in Uintah County, Utah, was completed in May 1980 and is operated primarily as an impoundment for irrigation water for the downstream community of Jensen, Utah as well as for Vernal, Utah. Red Fleet Reservoir can store up to approximately 26,000 acre-feet (acre-ft) of water at full stage. It also supplies water to the Ashley Valley Water Treatment Plant (AVWTP) in Vernal during spring runoff when Ashley Spring, the treatment plant's primary water source, has elevated turbidity. The reservoir is an on-channel impoundment of Big Brush Creek and has not been previously investigated with respect to the reservoir's interaction with the surrounding groundwater systems. The geology of the region was studied in the 1960s by the Bureau of Reclamation (BOR) to investigate the feasibility of future dam sites for Red Fleet Reservoir (Thompson, 1969). Extensive characterization of local geology around the reservoir site was attained by field mapping as well as multiple drilled test holes. The Uintah Water Conservancy District (UWCD) and the BOR are interested in evaluating the groundwater resources surrounding the reservoir as well as the surface-water/groundwater interactions that occur in Red Fleet Reservoir to better understand how the resources could be managed in the future if groundwater withdrawals were initiated. 
$109^{\circ} 35$ $109^{\circ} 30^{\prime}$ $109^{\circ} 25^{\circ}$

$109^{\circ} 20^{\prime}$

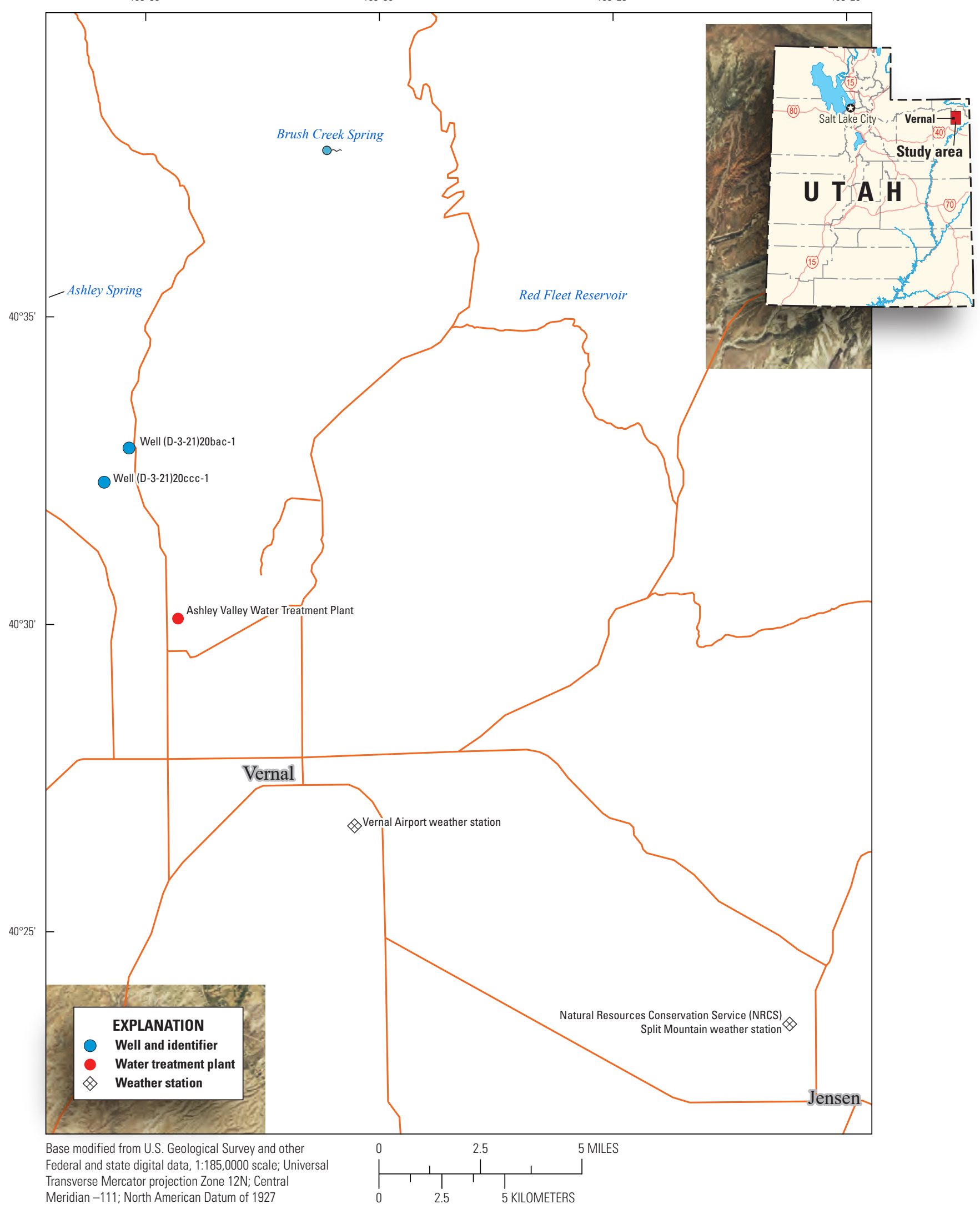

Figure 1. Location of the Red Fleet Reservoir study area, Uintah County, Utah. 
The objectives of this report are to present and interpret (1) groundwater levels, reservoir altitude, meteorological data, and inflows/outflows to and from Red Fleet Reservoir from May 1980 through December 2015 for the purpose of estimating groundwater recharge/discharge to and from surrounding aquifer units; (2) aquifer properties of the Nugget Sandstone near Red Fleet Reservoir from a two-well aquifer test; and (3) groundwater and surface-water chemical data to evaluate the origin of groundwater in aquifer units adjacent to Red Fleet Reservoir. This study is a cooperative effort by the UWCD, the BOR, and the U.S. Geological Survey (USGS).

\section{Hydrogeology}

Red Fleet Reservoir is on southward dipping Mesozoic lithologies ranging in age from Jurassic to Cretaceous. The geologic units dip southward at approximately 20-30 degrees. Formations crop out from north to south and include the Jurassic Nugget Sandstone, Carmel Formation, Entrada Sandstone, Curtis Formation, Morrison Formation, Cretaceous Dakota Sandstone, Mowry Shale, Frontier Sandstone, and Mancos Shale (Thompson, 1969). The Nugget Sandstone aquifer is utilized regionally for drinking water near Vernal, Utah. East/west jointing observed in the Frontier Sandstone may result in enhanced secondary permeability in relation to groundwater/surface-water interaction with Red Fleet Reservoir. Geologic units other than the Nugget Sandstone and Frontier Sandstone generally are characterized by fine-grained lithologies such as siltstones and shales and likely act as lowpermeability barriers to groundwater flow.

\section{Assessment of Groundwater Interaction with Red Fleet Reservoir}

To estimate groundwater interactions between Red Fleet Reservoir and the underlying sandstone aquifers, an annual surface-water budget for the reservoir was developed. The calculation of the reservoir water budget included reservoir inflows and outflows, changes in reservoir storage, evapotranspiration, and precipitation, with calculated residual values representing groundwater interactions with the reservoir. The reservoir water budget covers the length of time that Red Fleet Reservoir has been in operation (May 1980 through December 2015).

\section{Data Collection Methods and Results}

Many types of data have been collected as part of routine operations associated with Red Fleet Reservoir. These data include stream inflow from Big Brush Creek, stream outflow from dam releases and spillway discharge, reservoir and monitoring-well water levels, meteorological parameters, and pumpage from the Tyzack Pump Station to the nearby AVWTP and irrigation distribution systems (figs. 1 and 2).

\section{Surface-Water Inflow to and Outflow from Red Fleet Reservoir}

Big Brush Creek is the only perennial source of surfacewater inflow to Red Fleet Reservoir. Big Brush Creek is predominantly supplied by Brush Creek Spring, which is about 4 miles (mi) upstream of the U.S. Highway 191 bridge over Big Brush Creek (fig. 1). The spring is the primary discharge point for karst features that are developed in Paleozoic carbonate lithologies on the south slope of the Uinta Mountains. Other small ephemeral streams that drain into the reservoir, Cottonwood Wash being the largest, only contribute small amounts of discharge during spring runoff in above average precipitation years and during intense monsoonal precipitation events. The USGS has operated a streamgage on Big Brush Creek since 1939; from 1939 to 1979 the gage (USGS 09262000, now inundated by Red Fleet Reservoir) was located along the historic Donkey Flats Road where it crossed Big Brush Creek. As a result of the implementation of Red Fleet Reservoir, the streamgaging site for Big Brush Creek was moved upstream near U.S. Highway 191 where it crosses Big Brush Creek in order to avoid inundation. This USGS site (09261700) has been operated continuously since 1979.

From 1980 through 2015, Big Brush Creek discharged an average of 29,200 acre-feet per year (acre-ft/yr) of water to Red Fleet Reservoir. Maximum and minimum annual discharge from Big Brush Creek to Red Fleet Reservoir from 1980 through 2015 was 49,000 and 12,200 acre-ft, respectively, with the minimum year in 2002 and the maximum year in 1998 (fig. 3).

Streamflow discharge from Red Fleet Reservoir is controlled by gates and jet valves at the base of Red Fleet Dam as well as a spillway on the east abutment. The discharge works have been operated continuously by the BOR or the UWCD since the reservoir first started filling in May 1980. A record of daily discharge has been maintained in the form of a written $\log$ book from which the data for this study were obtained (John Hunting, Uintah Water Conservancy District, written commun., 2016). Water from the reservoir has only discharged over the spillway for a short period during aboveaverage spring runoff conditions in June 1984 and was not quantified in the water budget. For all other periods during the operational record of the dam, discharge is well quantified through the logged record of discharge gate and valve settings. 


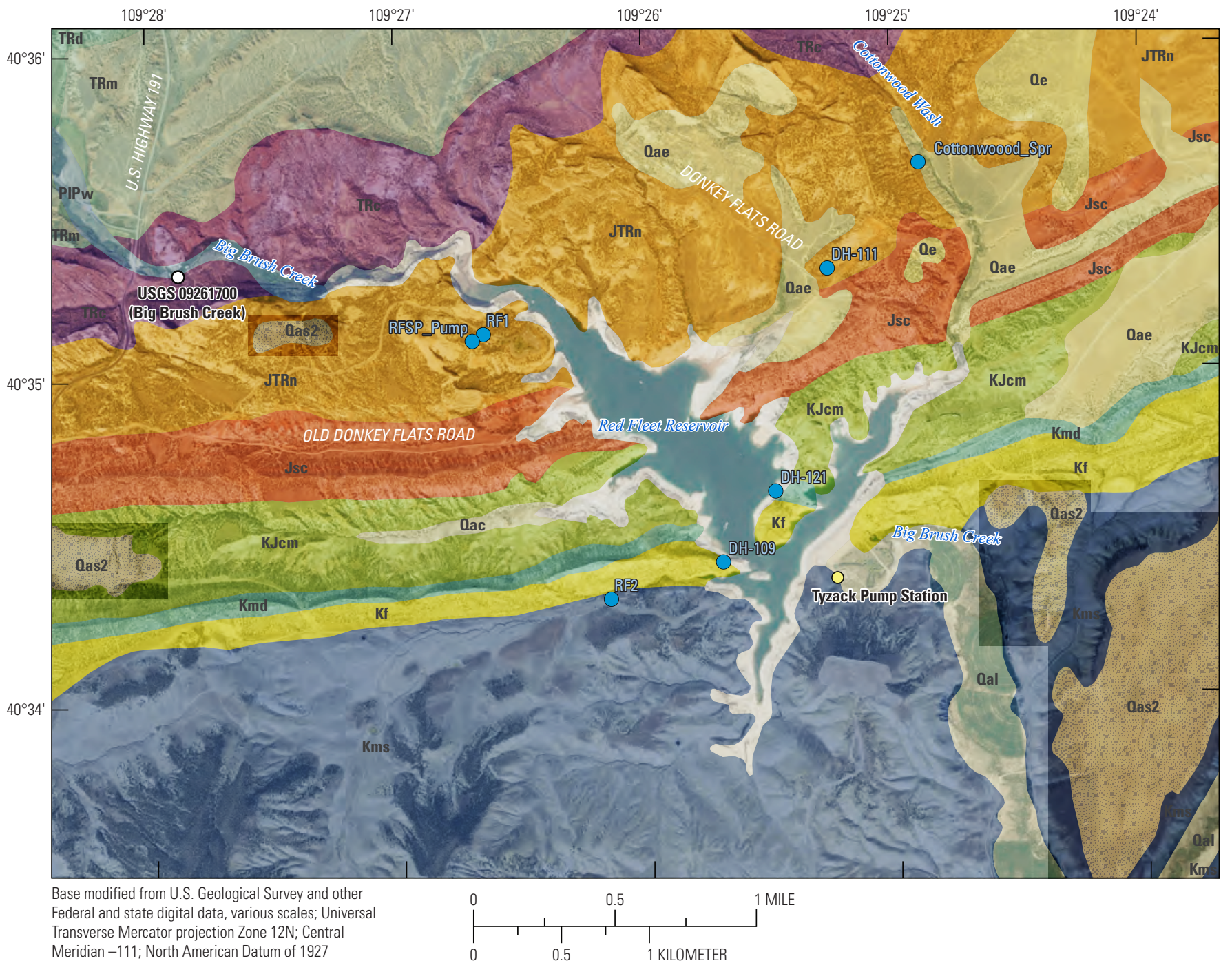

Meridian -111; North American Datum of 1927

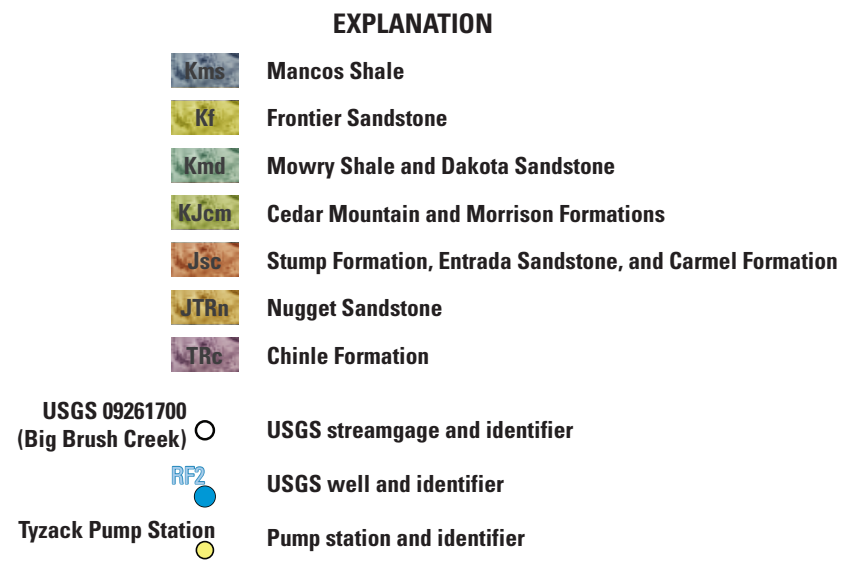

Figure 2. Geology and location of wells, streamgage, and pump station near Red Fleet Reservoir, Utah. 


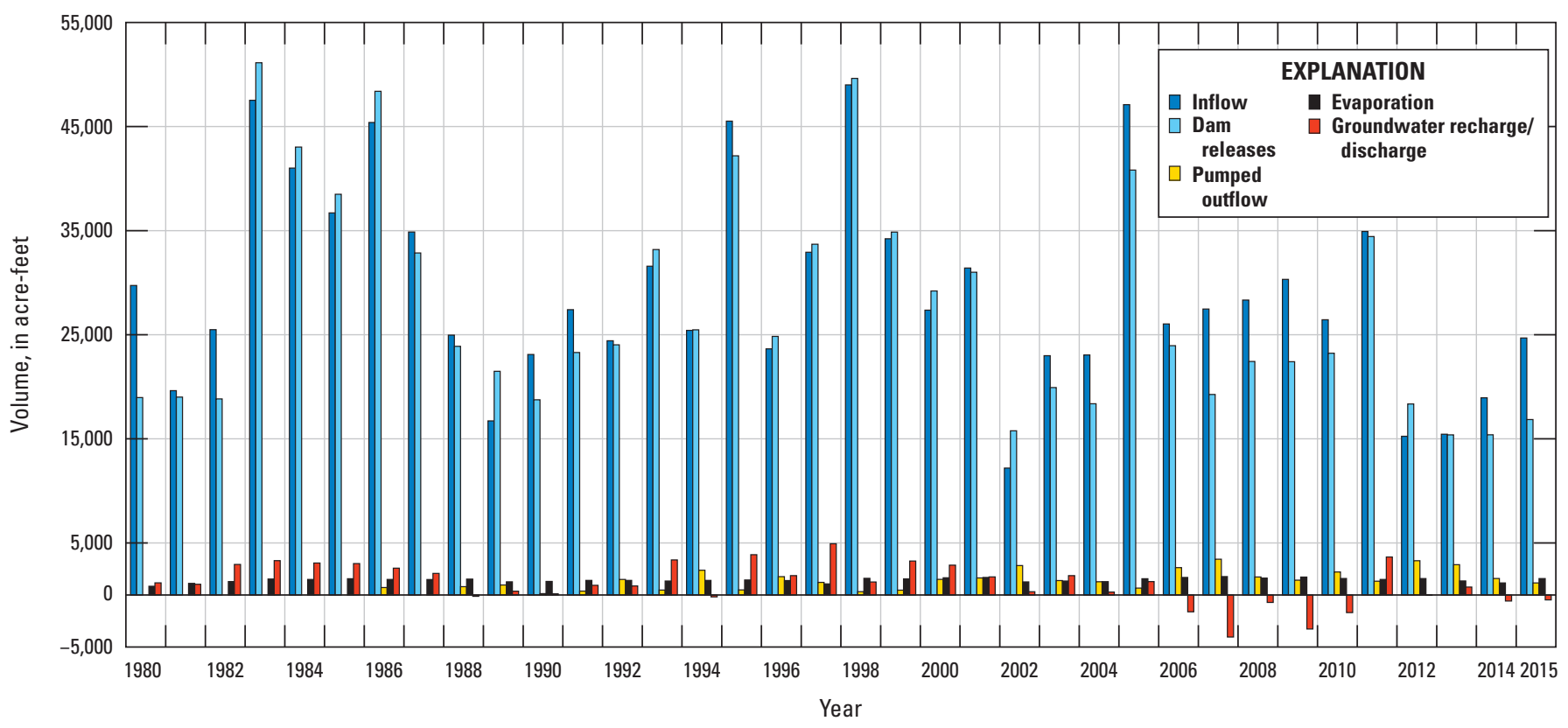

Figure 3. Annual inflows and outflows to and from Red Fleet Reservoir, near Vernal, Utah, 1980-2015.

\section{Pumped Outflow from Red Fleet Reservoir}

The Tyzack Pump Station is at the base of Red Fleet Dam and is operated by the UWCD (fig. 1). Pumping from the station first occurred during the early summer of 1986 to provide water to the AVWTP (Brad Grammar, Ashley Valley Water Treatment Plant, written commun., 2016). Pumping to provide water to the AVWTP occurred every early summer from 1988 to 2015. The average annual pumped volume of water from the Tyzack Pump Station during years when pumping occurred was about 1,400 acre-ft. The total volume of water pumped from the Tyzack Pump Station from 1980 through 2015 was about 42,500 acre-ft (table 1). Beginning in 1994, a diversion was built into the pipeline that connects the Tyzack Pump Station and AVWTP. The UWCD utilized this pipeline to supply irrigation water to users in Ashley Valley. This diversion was used in the summers of 1994, 1996, 1997, and every summer between 2000 and 2015. During the years when the irrigation diversion was used, the annual volume of water diverted from the pipeline for irrigation ranged from 58 to 1,078 acre-ft. 
Table 1. Reservoir data, evaporation, and calculated recharge and discharge from Red Fleet Reservoir, Utah, $1980-2015$.

[AVWTP, Ashley Valley Water Treatment Plant; $\sigma$, sigma (standard deviation); 一, no data]

\begin{tabular}{|c|c|c|c|c|c|c|c|c|c|c|c|c|c|c|c|}
\hline \multirow{2}{*}{ Month/year } & \multirow{2}{*}{$\begin{array}{l}\text { Reservoir } \\
\text { altitude } \\
\text { (feet) }\end{array}$} & \multirow{2}{*}{$\begin{array}{c}\text { Monthly } \\
\text { Big Brush } \\
\text { Creek } \\
\text { inflow } \\
\text { (acre-feet) }\end{array}$} & \multirow{2}{*}{$\begin{array}{l}\text { Monthly } \\
\text { Red Fleet } \\
\text { Dam out- } \\
\text { flow } \\
\text { (acre-feet) }\end{array}$} & \multicolumn{2}{|c|}{$\begin{array}{c}\text { Monthly pumped } \\
\text { outflows } \\
\text { (acre-feet) }\end{array}$} & \multirow{2}{*}{$\begin{array}{c}\text { Res- } \\
\text { ervoir } \\
\text { - storage } \\
\text { (acre- } \\
\text { feet) }\end{array}$} & \multirow{2}{*}{$\begin{array}{c}\text { Monthly } \\
\text { evapora- } \\
\text { tion } \\
\text { (acre- } \\
\text { feet) }\end{array}$} & \multirow{2}{*}{$\begin{array}{l}\text { Monthly } \\
\text { precipita- } \\
\text { tion } \\
\text { (acre- } \\
\text { feet) }\end{array}$} & \multirow{2}{*}{$\begin{array}{c}\text { Monthly } \\
\text { groundwater } \\
\text { recharge/ } \\
\text { discharge } \\
\text { (-) } \\
\text { (acre-feet) }\end{array}$} & \multirow{2}{*}{$\begin{array}{l}\text { Res- } \\
\text { ervoir } \\
\text { surface } \\
\text { area } \\
\text { (acres) }\end{array}$} & \multirow{2}{*}{$\begin{array}{l}\text { Monthly } \\
\text { evapora- } \\
\text { tion rate } \\
\text { (feet) }\end{array}$} & \multirow{2}{*}{$\begin{array}{l}\text { Monthly } \\
\text { precipita- } \\
\text { tion rate } \\
\text { (feet) }\end{array}$} & \multirow{2}{*}{$\begin{array}{l}\text { Monthly } \\
\text { reservoir } \\
\text { storage } \\
\text { change } \\
\text { (acre-feet) }\end{array}$} & \multirow{2}{*}{$\begin{array}{c}\text { Monthly } \\
\text { groundwater } \\
\text { recharge/ } \\
\text { discharge un- } \\
\text { certainty, } 2 \sigma \\
\text { (percent) }\end{array}$} & \multirow{2}{*}{$\begin{array}{c}\text { Monthly } \\
\text { groundwater } \\
\text { recharge/ } \\
\text { discharge un- } \\
\text { certainty, } 2 \sigma \\
\text { (acre-feet) }\end{array}$} \\
\hline & & & & AVWTP & $\begin{array}{l}\text { Irriga- } \\
\text { tion }\end{array}$ & & & & & & & & & & \\
\hline Empty stage & $5,494.2$ & & & & & 0 & & & & & & & & & \\
\hline May-80 & $5,561.6$ & 9,830 & 2,959 & 0 & 0 & 8,237 & 57 & 20 & $-1,403$ & 149 & 0.38 & 0.14 & $8,237.24$ & 10.04 & 140.78 \\
\hline June- 80 & $5,576.6$ & 11,080 & 5,899 & 0 & 0 & 12,700 & 191 & 0 & 528 & 321 & 0.59 & 0.00 & $4,462.29$ & 10.09 & 53.28 \\
\hline July-80 & $5,574.05$ & 2,690 & 3,491 & 0 & 0 & 11,866 & 236 & 16 & -187 & 333 & 0.71 & 0.05 & -833.80 & 10.35 & 19.37 \\
\hline August-80 & $5,569.95$ & 2,020 & 3,113 & 0 & 0 & 10,587 & 171 & 24 & 39 & 313 & 0.55 & 0.08 & $-1,279.08$ & 10.30 & 4.01 \\
\hline September- 80 & $5,569.4$ & 1,240 & 1,494 & 0 & 0 & 10,421 & 106 & 22 & -172 & 302 & 0.35 & 0.07 & -166.11 & 10.42 & 17.97 \\
\hline October- 80 & $5,567.7$ & 1,090 & 1,034 & 0 & 0 & 9,926 & 50 & 13 & 513 & 303 & 0.17 & 0.04 & -494.51 & 10.24 & 52.53 \\
\hline November- 80 & $5,571.4$ & 918 & 345 & 0 & 0 & 11,031 & 13 & 17 & -527 & 307 & 0.04 & 0.06 & $1,104.63$ & 10.13 & 53.39 \\
\hline December-80 & $5,571.98$ & 863 & 632 & 0 & 0 & 11,211 & 8 & 0 & 43 & 314 & 0.03 & 0.00 & 180.12 & 10.05 & 4.29 \\
\hline January-81 & $5,572.55$ & 710 & 535 & 0 & 0 & 11,389 & 5 & 3 & -6 & 315 & 0.02 & 0.01 & 178.45 & 10.05 & 0.59 \\
\hline February-81 & $5,573.78$ & 639 & 227 & 0 & 0 & 11,779 & 13 & 4 & 13 & 320 & 0.04 & 0.01 & 389.98 & 10.13 & 1.35 \\
\hline March-81 & $5,575.81$ & 718 & 184 & 0 & 0 & 12,438 & 40 & 43 & -122 & 330 & 0.12 & 0.13 & 658.73 & 10.50 & 12.84 \\
\hline April-81 & $5,579.45$ & 2,310 & 1,163 & 0 & 0 & 13,669 & 96 & 27 & -153 & 343 & 0.28 & 0.08 & $1,230.58$ & 10.25 & 15.66 \\
\hline May-81 & $5,580.6$ & 4,380 & 4,165 & 0 & 0 & 14,071 & 135 & 44 & -279 & 358 & 0.38 & 0.12 & 402.58 & 10.20 & 28.47 \\
\hline June-81 & $5,579.4$ & 3,370 & 3,807 & 0 & 0 & 13,651 & 223 & 2 & -237 & 359 & 0.62 & 0.01 & -419.93 & 10.29 & 24.42 \\
\hline July-81 & $5,574.69$ & 2,350 & 3,571 & 0 & 0 & 12,072 & 241 & 16 & 133 & 342 & 0.70 & 0.05 & $-1,579.03$ & 10.33 & 13.77 \\
\hline August-81 & $5,570.3$ & 1,550 & 2,792 & 0 & 0 & 10,693 & 177 & 19 & -20 & 314 & 0.56 & 0.06 & $-1,379.13$ & 10.33 & 2.08 \\
\hline September-81 & $5,568.2$ & 1,070 & 1,734 & 0 & 0 & 10,062 & 114 & 24 & -124 & 300 & 0.38 & 0.08 & -630.60 & 10.39 & 12.88 \\
\hline October-81 & $5,570.3$ & 914 & 593 & 0 & 0 & 10,693 & 46 & 109 & -247 & 299 & 0.15 & 0.36 & 630.60 & 10.68 & 26.35 \\
\hline November-81 & $5,572.7$ & 863 & 119 & 0 & 0 & 11,436 & 16 & 8 & -7 & 312 & 0.05 & 0.03 & 743.41 & 10.13 & 0.75 \\
\hline December-81 & $5,574.6$ & 750 & 123 & 0 & 0 & 12,043 & 5 & 11 & 27 & 323 & 0.01 & 0.03 & 606.58 & 10.10 & 2.68 \\
\hline January-82 & $5,577.1$ & 700 & 97 & 0 & 0 & 12,867 & 0 & 12 & -209 & 334 & 0.00 & 0.04 & 823.63 & 10.08 & 21.02 \\
\hline February-82 & $5,579.2$ & 604 & 0 & 0 & 0 & 13,582 & 12 & 5 & -118 & 341 & 0.04 & 0.02 & 715.27 & 10.13 & 11.97 \\
\hline March-82 & $5,581.2$ & 666 & 0 & 0 & 0 & 14,284 & 39 & 40 & -35 & 351 & 0.11 & 0.11 & 701.96 & 10.54 & 3.71 \\
\hline April-82 & $5,583.84$ & 1,050 & 359 & 0 & 0 & 15,243 & 69 & 7 & -330 & 370 & 0.19 & 0.02 & 958.93 & 10.31 & 34.00 \\
\hline May-82 & $5,595.94$ & 8,090 & 3,980 & 0 & 0 & 20,157 & 151 & 33 & -922 & 384 & 0.39 & 0.09 & $4,914.45$ & 10.11 & 93.18 \\
\hline June- 82 & $5,596.03$ & 3,430 & 3,612 & 0 & 0 & 20,188 & 248 & 8 & -453 & 447 & 0.55 & 0.02 & 31.11 & 10.35 & 46.89 \\
\hline July-82 & $5,593.76$ & 3,070 & 4,117 & 0 & 0 & 19,202 & 297 & 26 & -332 & 440 & 0.68 & 0.06 & -986.39 & 10.38 & 34.49 \\
\hline
\end{tabular}


Table 1. Reservoir data, evaporation, and calculated recharge and discharge from Red Fleet Reservoir, Utah, 1980-2015. - Continued

[AVWTP, Ashley Valley Water Treatment Plant; $\sigma$, sigma (standard deviation); - , no data]

\begin{tabular}{|c|c|c|c|c|c|c|c|c|c|c|c|c|c|c|c|}
\hline \multirow{2}{*}{ Month/year } & \multirow{2}{*}{$\begin{array}{c}\text { Reservoir } \\
\text { altitude } \\
\text { (feet) }\end{array}$} & \multirow{2}{*}{$\begin{array}{l}\text { Monthly } \\
\text { Big Brush } \\
\text { Creek } \\
\text { inflow } \\
\text { (acre-feet) }\end{array}$} & \multirow{2}{*}{$\begin{array}{c}\text { Monthly } \\
\text { Red Fleet } \\
\text { Dam out- } \\
\text { flow } \\
\text { (acre-feet) }\end{array}$} & \multicolumn{2}{|c|}{$\begin{array}{l}\text { Monthly pumped } \\
\text { outflows } \\
\text { (acre-feet) }\end{array}$} & \multirow{2}{*}{ 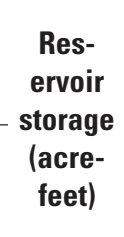 } & \multirow{2}{*}{$\begin{array}{c}\text { Monthly } \\
\text { evapora- } \\
\text { tion } \\
\text { (acre- } \\
\text { feet) }\end{array}$} & \multirow{2}{*}{$\begin{array}{l}\text { Monthly } \\
\text { precipita- } \\
\text { tion } \\
\text { (acre- } \\
\text { feet) }\end{array}$} & \multirow{2}{*}{$\begin{array}{c}\text { Monthly } \\
\text { groundwater } \\
\text { recharge/ } \\
\text { discharge } \\
\text { (-) } \\
\text { (acre-feet) }\end{array}$} & \multirow{2}{*}{$\begin{array}{l}\text { Res- } \\
\text { ervoir } \\
\text { surface } \\
\text { area } \\
\text { (acres) }\end{array}$} & \multirow{2}{*}{$\begin{array}{c}\text { Monthly } \\
\text { evapora- } \\
\text { tion rate } \\
\text { (feet) }\end{array}$} & \multirow{2}{*}{$\begin{array}{c}\text { Monthly } \\
\text { precipita- } \\
\text { tion rate } \\
\text { (feet) }\end{array}$} & \multirow{2}{*}{$\begin{array}{c}\text { Monthly } \\
\text { reservoir } \\
\text { storage } \\
\text { change } \\
\text { (acre-feet) }\end{array}$} & \multirow{2}{*}{$\begin{array}{l}\text { Monthly } \\
\text { groundwater } \\
\text { recharge/ } \\
\text { discharge un- } \\
\text { certainty, } 2 \sigma \\
\text { (percent) }\end{array}$} & \multirow{2}{*}{$\begin{array}{c}\text { Monthly } \\
\text { groundwater } \\
\text { recharge/ } \\
\text { discharge un- } \\
\text { certainty, } 2 \sigma \\
\text { (acre-feet) }\end{array}$} \\
\hline & & & & AVWTP & $\begin{array}{l}\text { Irriga- } \\
\text { tion }\end{array}$ & & & & & & & & & & \\
\hline August-82 & $5,590.58$ & 1,940 & 3,281 & 0 & 0 & 17,871 & 251 & 22 & -239 & 423 & 0.59 & 0.05 & $-1,330.88$ & 10.40 & 24.86 \\
\hline September-82 & $5,590.94$ & 1,600 & 1,494 & 0 & 0 & 18,019 & 144 & 93 & -93 & 414 & 0.35 & 0.23 & 147.71 & 10.68 & 9.93 \\
\hline October- 82 & $5,595.53$ & 1,860 & 192 & 0 & 0 & 19,968 & 61 & 65 & -278 & 442 & 0.14 & 0.15 & $1,949.66$ & 10.31 & 28.69 \\
\hline November- 82 & $5,597.15$ & 1,270 & 486 & 0 & 0 & 20,686 & 13 & 35 & 89 & 451 & 0.03 & 0.08 & 717.90 & 10.19 & 9.07 \\
\hline December-82 & $5,597.25$ & 1,210 & 1,214 & 0 & 0 & 20,731 & 3 & 37 & -14 & 454 & 0.01 & 0.08 & 44.83 & 10.16 & 1.46 \\
\hline January-83 & $5,597.4$ & 1,070 & 1,202 & 0 & 0 & 20,799 & 2 & 21 & -181 & 455 & 0.00 & 0.05 & 67.36 & 10.10 & 18.23 \\
\hline February-83 & $5,597.47$ & 859 & 502 & 0 & 0 & 20,830 & 11 & 36 & 350 & 457 & 0.02 & 0.08 & 31.48 & 10.33 & 36.16 \\
\hline March-83 & $5,600.72$ & 1,050 & 419 & 0 & 0 & 22,324 & 61 & 29 & -896 & 472 & 0.13 & 0.06 & $1,494.36$ & 10.29 & 92.21 \\
\hline April-83 & $5,597.83$ & 1,130 & 2,627 & 0 & 0 & 20,992 & 78 & 51 & -192 & 457 & 0.17 & 0.11 & $-1,332.00$ & 10.25 & 19.64 \\
\hline May-83 & $5,602.43$ & 6,850 & 4,990 & 0 & 0 & 23,137 & 160 & 52 & -392 & 460 & 0.35 & 0.11 & $2,144.29$ & 10.15 & 39.81 \\
\hline June- 83 & $5,608.02$ & 18,680 & 16,652 & 0 & 0 & 25,920 & 283 & 39 & -998 & 520 & 0.54 & 0.08 & $2,783.37$ & 10.08 & 100.67 \\
\hline July-83 & $5,606.75$ & 7,780 & 8,567 & 0 & 0 & 25,270 & 343 & 30 & -450 & 521 & 0.66 & 0.06 & -649.77 & 10.21 & 46.00 \\
\hline August-83 & $5,605.03$ & 3,150 & 4,271 & 0 & 0 & 24,407 & 306 & 29 & -535 & 511 & 0.60 & 0.06 & -863.60 & 10.39 & 55.54 \\
\hline September-83 & $5,600.55$ & 1,760 & 3,865 & 0 & 0 & 22,245 & 182 & 33 & -92 & 487 & 0.37 & 0.07 & $-2,162.06$ & 10.27 & 9.42 \\
\hline October- 83 & $5,596.4$ & 2,070 & 3,612 & 0 & 0 & 20,352 & 82 & 32 & 300 & 462 & 0.18 & 0.07 & $-1,892.58$ & 10.15 & 30.40 \\
\hline November- 83 & $5,596.65$ & 1,570 & 1,965 & 0 & 0 & 20,463 & 19 & 57 & -469 & 449 & 0.04 & 0.13 & 111.06 & 10.21 & 47.82 \\
\hline December-83 & $5,594.1$ & 1,560 & 2,460 & 0 & 0 & 19,348 & 0 & 42 & 257 & 443 & 0.00 & 0.09 & $-1,115.34$ & 10.08 & 25.94 \\
\hline January-84 & $5,591.7$ & 1,380 & 2,460 & 0 & 0 & 18,333 & 0 & 11 & -54 & 428 & 0.00 & 0.03 & $-1,014.66$ & 10.02 & 5.39 \\
\hline February-84 & $5,591.3$ & 1,150 & 1,320 & 0 & 0 & 18,167 & 0 & 8 & 4 & 419 & 0.00 & 0.02 & -165.84 & 10.03 & 0.37 \\
\hline March-84 & $5,591.14$ & 1,160 & 1,355 & 0 & 0 & 18,101 & 29 & 12 & -146 & 418 & 0.07 & 0.03 & -66.08 & 10.16 & 14.84 \\
\hline April-84 & $5,591.35$ & 1,730 & 1,696 & 0 & 0 & 18,188 & 80 & 25 & -108 & 418 & 0.19 & 0.06 & 86.76 & 10.29 & 11.13 \\
\hline May-84 & $5,605.78$ & 11,150 & 4,953 & 0 & 0 & 24,781 & 197 & 15 & -579 & 448 & 0.44 & 0.03 & $6,592.99$ & 10.09 & 58.39 \\
\hline June-84 & $5,608.1$ & 11,620 & 11,388 & 0 & 0 & 25,961 & 289 & 66 & $-1,171$ & 528 & 0.55 & 0.12 & $1,180.39$ & 10.14 & 118.79 \\
\hline July-84 & $5,605.32$ & 3,460 & 5,621 & 0 & 0 & 24,551 & 361 & 51 & $-1,061$ & 519 & 0.70 & 0.10 & $-1,410.36$ & 10.38 & 110.06 \\
\hline August-84 & $5,602.07$ & 2,790 & 4,545 & 0 & 0 & 22,964 & 284 & 16 & -435 & 495 & 0.57 & 0.03 & $-1,586.80$ & 10.32 & 44.95 \\
\hline September- 84 & $5,598.73$ & 1,770 & 3,410 & 0 & 0 & 21,402 & 167 & 42 & -202 & 476 & 0.35 & 0.09 & $-1,562.42$ & 10.30 & 20.82 \\
\hline October-84 & $5,595.3$ & 1,940 & 3,083 & 0 & 0 & 19,868 & 66 & 39 & 364 & 449 & 0.15 & 0.09 & $-1,533.94$ & 10.16 & 36.99 \\
\hline November-84 & $5,594.2$ & 1,520 & 2,380 & 0 & 0 & 19,391 & 16 & 25 & -374 & 442 & 0.04 & 0.06 & -477.04 & 10.09 & 37.77 \\
\hline
\end{tabular}




\begin{tabular}{|c|c|c|c|c|c|c|c|c|c|c|c|c|c|c|c|}
\hline \multirow{2}{*}{ Month/year } & \multirow{2}{*}{$\begin{array}{l}\text { Reservoir } \\
\text { altitude } \\
\text { (feet) }\end{array}$} & \multirow{2}{*}{$\begin{array}{c}\text { Monthly } \\
\text { Big Brush } \\
\text { Creek } \\
\text { inflow } \\
\text { (acre-feet) }\end{array}$} & \multirow{2}{*}{$\begin{array}{l}\text { Monthly } \\
\text { Red Fleet } \\
\text { Dam out- } \\
\text { flow } \\
\text { (acre-feet) }\end{array}$} & \multicolumn{2}{|c|}{$\begin{array}{c}\text { Monthly pumped } \\
\text { outflows } \\
\text { (acre-feet) }\end{array}$} & \multirow{2}{*}{$\begin{array}{c}\text { Res- } \\
\text { ervoir } \\
\text { storage } \\
\text { (acre- } \\
\text { feet) }\end{array}$} & \multirow{2}{*}{$\begin{array}{c}\text { Monthly } \\
\text { evapora- } \\
\text { tion } \\
\text { (acre- } \\
\text { feet) }\end{array}$} & \multirow{2}{*}{$\begin{array}{c}\text { Monthly } \\
\text { precipita- } \\
\text { tion } \\
\text { (acre- } \\
\text { feet) }\end{array}$} & \multirow{2}{*}{$\begin{array}{c}\text { Monthly } \\
\text { groundwater } \\
\text { recharge/ } \\
\text { discharge } \\
\text { (-) } \\
\text { (acre-feet) }\end{array}$} & \multirow{2}{*}{$\begin{array}{l}\text { Res- } \\
\text { ervoir } \\
\text { surface } \\
\text { area } \\
\text { (acres) }\end{array}$} & \multirow{2}{*}{$\begin{array}{c}\text { Monthly } \\
\text { evapora- } \\
\text { tion rate } \\
\text { (feet) }\end{array}$} & \multirow{2}{*}{$\begin{array}{c}\text { Monthly } \\
\text { precipita- } \\
\text { tion rate } \\
\text { (feet) }\end{array}$} & \multirow{2}{*}{$\begin{array}{l}\text { Monthly } \\
\text { reservoir } \\
\text { storage } \\
\text { change } \\
\text { (acre-feet) }\end{array}$} & \multirow{2}{*}{$\begin{array}{c}\text { Monthly } \\
\text { groundwater } \\
\text { recharge/ } \\
\text { discharge un- } \\
\text { certainty, } 2 \sigma \\
\text { (percent) }\end{array}$} & \multirow{2}{*}{$\begin{array}{c}\text { Monthly } \\
\text { groundwater } \\
\text { recharge/ } \\
\text { discharge un- } \\
\text { certainty, } 2 \sigma \\
\text { (acre-feet) }\end{array}$} \\
\hline & & & & AVWTP & $\begin{array}{c}\text { Irriga- } \\
\text { tion }\end{array}$ & & & & & & & & & & \\
\hline December-84 & $5,593.9$ & 1,340 & 821 & 0 & 0 & 19,262 & 1 & 43 & 691 & 435 & 0.00 & 0.10 & -128.86 & 10.19 & 70.42 \\
\hline January-85 & 5,596 & 1,160 & 325 & 0 & 0 & 20,175 & 0 & 13 & -65 & 445 & 0.00 & 0.03 & 913.21 & 10.05 & 6.57 \\
\hline February-85 & $5,596.4$ & 974 & 1,055 & 0 & 0 & 20,352 & 1 & 7 & -252 & 449 & 0.00 & 0.02 & 176.92 & 10.03 & 25.29 \\
\hline March-85 & $5,596.4$ & 1,120 & 1,168 & 0 & 0 & 20,352 & 35 & 31 & -52 & 449 & 0.08 & 0.07 & 0.00 & 10.28 & 5.36 \\
\hline April-85 & $5,602.4$ & 5,290 & 2,530 & 0 & 0 & 23,122 & 126 & 15 & -121 & 474 & 0.26 & 0.03 & $2,770.23$ & 10.13 & 12.22 \\
\hline May-85 & $5,607.94$ & 12,870 & 11,243 & 0 & 0 & 25,879 & 223 & 35 & $-1,318$ & 517 & 0.43 & 0.07 & $2,756.54$ & 10.10 & 133.01 \\
\hline June-85 & 5,607 & 3,920 & 4,692 & 0 & 0 & 25,397 & 315 & 41 & -565 & 522 & 0.60 & 0.08 & -481.45 & 10.38 & 58.64 \\
\hline July-85 & $5,605.5$ & 3,060 & 3,775 & 0 & 0 & 24,641 & 357 & 47 & -269 & 507 & 0.70 & 0.09 & -756.57 & 10.51 & 28.23 \\
\hline August-85 & $5,602.1$ & 2,390 & 4,109 & 0 & 0 & 22,979 & 275 & 2 & -329 & 498 & 0.55 & 0.00 & $-1,662.28$ & 10.33 & 34.02 \\
\hline September-85 & $5,599.05$ & 1,860 & 3,343 & 0 & 0 & 21,548 & 143 & 74 & -122 & 474 & 0.30 & 0.16 & $-1,430.04$ & 10.32 & 12.60 \\
\hline October- 85 & $5,593.4$ & 1,660 & 3,997 & 0 & 0 & 19,048 & 68 & 37 & 132 & 450 & 0.15 & 0.08 & $-2,500.14$ & 10.13 & 13.40 \\
\hline November-85 & $5,593.18$ & 1,270 & 1,398 & 0 & 0 & 18,955 & 15 & 37 & -13 & 430 & 0.03 & 0.09 & -93.51 & 10.18 & 1.28 \\
\hline December-85 & $5,593.95$ & 1,130 & 856 & 0 & 0 & 19,283 & 1 & 15 & -40 & 430 & 0.00 & 0.04 & 328.53 & 10.07 & 4.03 \\
\hline January- 86 & $5,595.86$ & 1,130 & 307 & 0 & 0 & 20,113 & 0 & 7 & -1 & 440 & 0.00 & 0.02 & 830.07 & 10.03 & 0.14 \\
\hline February-86 & $5,597.9$ & 1,050 & 278 & 0 & 0 & 21,024 & 24 & 14 & -149 & 453 & 0.05 & 0.03 & 910.63 & 10.17 & 15.17 \\
\hline March-86 & 5,598 & 1,500 & 1,302 & 0 & 0 & 21,069 & 76 & 42 & 118 & 461 & 0.17 & 0.09 & 45.28 & 10.40 & 12.32 \\
\hline April-86 & $5,595.19$ & 4,820 & 6,183 & 0 & 0 & 19,820 & 98 & 83 & -129 & 449 & 0.22 & 0.18 & $-1,249.56$ & 10.15 & 13.05 \\
\hline May-86 & $5,604.05$ & 12,850 & 8,792 & 287 & 0 & 23,923 & 169 & 47 & -453 & 468 & 0.36 & 0.10 & $4,103.22$ & 10.08 & 45.71 \\
\hline June-86 & $5,607.81$ & 11,120 & 10,006 & 334 & 0 & 25,812 & 312 & 20 & $-1,400$ & 520 & 0.60 & 0.04 & $1,888.89$ & 10.14 & 142.01 \\
\hline July-86 & $5,605.22$ & 3,120 & 4,552 & 0 & 0 & 24,501 & 317 & 43 & -396 & 510 & 0.62 & 0.08 & $-1,310.74$ & 10.38 & 41.12 \\
\hline August-86 & $5,600.7$ & 2,410 & 4,422 & 17 & 0 & 22,315 & 268 & 49 & -61 & 490 & 0.55 & 0.10 & $-2,186.16$ & 10.34 & 6.28 \\
\hline September- 86 & $5,596.22$ & 1,940 & 3,746 & 76 & 0 & 20,272 & 138 & 74 & 96 & 459 & 0.30 & 0.16 & $-2,042.69$ & 10.26 & 9.89 \\
\hline October-86 & $5,594.4$ & 2,350 & 3,074 & 0 & 0 & 19,477 & 67 & 61 & 65 & 443 & 0.15 & 0.14 & -795.34 & 10.20 & 6.59 \\
\hline November-86 & $5,592.47$ & 1,740 & 2,680 & 0 & 0 & 18,655 & 18 & 3 & -133 & 434 & 0.04 & 0.01 & -822.00 & 10.04 & 13.35 \\
\hline December-86 & $5,588.67$ & 1,360 & 3,043 & 0 & 0 & 17,100 & 3 & 5 & -126 & 414 & 0.01 & 0.01 & $-1,555.12$ & 10.01 & 12.63 \\
\hline January-87 & $5,589.09$ & 1,290 & 989 & 0 & 0 & 17,266 & 2 & 13 & 147 & 404 & 0.00 & 0.03 & 165.93 & 10.06 & 14.75 \\
\hline February-87 & $5,589.82$ & 1,190 & 833 & 0 & 0 & 17,560 & 12 & 27 & 78 & 409 & 0.03 & 0.07 & 294.13 & 10.17 & 7.97 \\
\hline March-87 & $5,590.5$ & 1,280 & 922 & 0 & 0 & 17,837 & 36 & 36 & 81 & 413 & 0.09 & 0.09 & 276.90 & 10.28 & 8.37 \\
\hline
\end{tabular}


Table 1. Reservoir data, evaporation, and calculated recharge and discharge from Red Fleet Reservoir, Utah, 1980-2015. - Continued

[AVWTP, Ashley Valley Water Treatment Plant; $\sigma$, sigma (standard deviation); - , no data]

\begin{tabular}{|c|c|c|c|c|c|c|c|c|c|c|c|c|c|c|c|}
\hline \multirow{2}{*}{ Month/year } & \multirow{2}{*}{$\begin{array}{l}\text { Reservoir } \\
\text { altitude } \\
\text { (feet) }\end{array}$} & \multirow{2}{*}{$\begin{array}{c}\text { Monthly } \\
\text { Big Brush } \\
\text { Creek } \\
\text { inflow } \\
\text { (acre-feet) }\end{array}$} & \multirow{2}{*}{$\begin{array}{c}\text { Monthly } \\
\text { Red Fleet } \\
\text { Dam out- } \\
\text { flow } \\
\text { (acre-feet) }\end{array}$} & \multicolumn{2}{|c|}{$\begin{array}{c}\text { Monthly pumped } \\
\text { outflows } \\
\text { (acre-feet) }\end{array}$} & \multirow{2}{*}{$\begin{array}{c}\text { Res- } \\
\text { ervoir } \\
\text { - storage } \\
\text { (acre- } \\
\text { feet) }\end{array}$} & \multirow{2}{*}{$\begin{array}{c}\text { Monthly } \\
\text { evapora- } \\
\text { tion } \\
\text { (acre- } \\
\text { feet) }\end{array}$} & \multirow{2}{*}{$\begin{array}{c}\text { Monthly } \\
\text { precipita- } \\
\text { tion } \\
\text { (acre- } \\
\text { feet) }\end{array}$} & \multirow{2}{*}{$\begin{array}{c}\text { Monthly } \\
\text { groundwater } \\
\text { recharge/ } \\
\text { discharge } \\
\text { (-) } \\
\text { (acre-feet) }\end{array}$} & \multirow{2}{*}{$\begin{array}{l}\text { Res- } \\
\text { ervoir } \\
\text { surface } \\
\text { area } \\
\text { (acres) }\end{array}$} & \multirow{2}{*}{$\begin{array}{c}\text { Monthly } \\
\text { evapora- } \\
\text { tion rate } \\
\text { (feet) }\end{array}$} & \multirow{2}{*}{$\begin{array}{c}\text { Monthly } \\
\text { precipita- } \\
\text { tion rate } \\
\text { (feet) }\end{array}$} & \multirow{2}{*}{$\begin{array}{c}\text { Monthly } \\
\text { reservoir } \\
\text { storage } \\
\text { change } \\
\text { (acre-feet) }\end{array}$} & \multirow{2}{*}{$\begin{array}{c}\text { Monthly } \\
\text { groundwater } \\
\text { recharge/ } \\
\text { discharge un- } \\
\text { certainty, } 2 \sigma \\
\text { (percent) }\end{array}$} & \multirow{2}{*}{$\begin{array}{c}\text { Monthly } \\
\text { groundwater } \\
\text { recharge/ } \\
\text { discharge un } \\
\text { certainty, } 2 \sigma \\
\text { (acre-feet) }\end{array}$} \\
\hline & & & & AVWTP & $\begin{array}{l}\text { Irriga- } \\
\text { tion }\end{array}$ & & & & & & & & & & \\
\hline April-87 & $5,597.45$ & 4,230 & 1,286 & 0 & 0 & 20,821 & 104 & 2 & -141 & 427 & 0.24 & 0.01 & $2,984.22$ & 10.12 & 14.32 \\
\hline May-87 & $5,606.81$ & 12,180 & 8,401 & 0 & 0 & 25,301 & 204 & 63 & -842 & 507 & 0.40 & 0.12 & $4,479.71$ & 10.11 & 85.10 \\
\hline June-87 & $5,606.19$ & 4,610 & 5,352 & 0 & 0 & 24,987 & 305 & 11 & -722 & 519 & 0.59 & 0.02 & -313.70 & 10.30 & 74.35 \\
\hline July-87 & $5,603.23$ & 2,860 & 4,481 & 0 & 0 & 23,523 & 316 & 84 & -390 & 498 & 0.63 & 0.17 & $-1,464.10$ & 10.43 & 40.66 \\
\hline August-87 & $5,600.42$ & 2,100 & 3,742 & 0 & 0 & 22,184 & 247 & 67 & -483 & 489 & 0.51 & 0.14 & $-1,339.20$ & 10.42 & 50.33 \\
\hline September-87 & $5,596.31$ & 1,630 & 3,570 & 0 & 0 & 20,312 & 157 & 5 & -221 & 462 & 0.34 & 0.01 & $-1,871.59$ & 10.22 & 22.61 \\
\hline October-87 & 5,595 & 1,250 & 1,743 & 0 & 0 & 19,737 & 80 & 56 & 58 & 441 & 0.18 & 0.13 & -575.16 & 10.37 & 6.01 \\
\hline November-87 & $5,595.35$ & 1,230 & 893 & 0 & 0 & 19,890 & 19 & 45 & 211 & 441 & 0.04 & 0.10 & 152.67 & 10.27 & 21.63 \\
\hline December-87 & $5,595.88$ & 1,020 & 648 & 0 & 0 & 20,122 & 3 & 21 & 157 & 444 & 0.01 & 0.05 & 232.57 & 10.12 & 15.86 \\
\hline January- 88 & $5,596.44$ & 946 & 615 & 0 & 0 & 20,370 & 0 & 13 & 97 & 447 & 0.00 & 0.03 & 247.56 & 10.07 & 9.77 \\
\hline February-88 & $5,596.88$ & 831 & 575 & 0 & 0 & 20,566 & 3 & 3 & 60 & 451 & 0.01 & 0.01 & 195.83 & 10.04 & 5.99 \\
\hline March-88 & $5,597.33$ & 986 & 615 & 0 & 0 & 20,767 & 49 & 8 & 129 & 453 & 0.11 & 0.02 & 201.48 & 10.31 & 13.26 \\
\hline April-88 & $5,601.28$ & 3,020 & 931 & 0 & 0 & 22,588 & 127 & 62 & 201 & 464 & 0.27 & 0.13 & $1,821.32$ & 10.32 & 20.78 \\
\hline May-88 & $5,608.08$ & 8,140 & 4,946 & 0 & 0 & 25,951 & 209 & 36 & -342 & 509 & 0.41 & 0.07 & $3,362.59$ & 10.15 & 34.74 \\
\hline June- 88 & $5,604.95$ & 3,260 & 5,023 & 0 & 0 & 24,367 & 312 & 18 & -474 & 516 & 0.61 & 0.04 & $-1,584.04$ & 10.32 & 48.90 \\
\hline July- 88 & $5,599.95$ & 2,300 & 4,676 & 49 & 0 & 21,965 & 332 & 7 & -348 & 486 & 0.68 & 0.01 & $-2,402.46$ & 10.35 & 36.01 \\
\hline August-88 & $5,593.47$ & 1,780 & 3,856 & 425 & 0 & 19,078 & 244 & 10 & 151 & 451 & 0.54 & 0.02 & $-2,886.37$ & 10.28 & 15.54 \\
\hline September- 88 & $5,590.98$ & 1,060 & 1,530 & 218 & 0 & 18,035 & 138 & 29 & 246 & 422 & 0.33 & 0.07 & $-1,042.88$ & 10.42 & 25.57 \\
\hline October- 88 & $5,590.85$ & 904 & 505 & 109 & 0 & 17,982 & 84 & 0 & 259 & 416 & 0.20 & 0.00 & -53.46 & 10.51 & 27.26 \\
\hline November- 88 & $5,591.69$ & 807 & 369 & 0 & 0 & 18,329 & 18 & 5 & 78 & 418 & 0.04 & 0.01 & 347.15 & 10.15 & 7.95 \\
\hline December-88 & $5,593.15$ & 926 & 246 & 0 & 0 & 18,942 & 1 & 8 & 74 & 425 & 0.00 & 0.02 & 613.14 & 10.05 & 7.46 \\
\hline January-89 & $5,594.6$ & 910 & 246 & 0 & 0 & 19,563 & 0 & 9 & 51 & 434 & 0.00 & 0.02 & 621.32 & 10.05 & 5.17 \\
\hline February-89 & $5,595.82$ & 752 & 222 & 0 & 0 & 20,096 & 3 & 4 & -1 & 442 & 0.01 & 0.01 & 532.41 & 10.05 & 0.15 \\
\hline March-89 & $5,597.31$ & 976 & 246 & 0 & 0 & 20,758 & 65 & 8 & 10 & 450 & 0.15 & 0.02 & 662.29 & 10.37 & 1.06 \\
\hline April-89 & $5,599.92$ & 3,290 & 2,416 & 0 & 0 & 21,951 & 123 & 2 & -439 & 462 & 0.27 & 0.01 & $1,192.45$ & 10.18 & 44.68 \\
\hline May-89 & $5,596.53$ & 3,120 & 4,900 & 0 & 0 & 20,410 & 187 & 10 & -417 & 460 & 0.41 & 0.02 & $-1,540.81$ & 10.20 & 42.53 \\
\hline June-89 & $5,591.06$ & 1,590 & 3,662 & 120 & 0 & 18,068 & 236 & 45 & -41 & 435 & 0.54 & 0.10 & $-2,341.54$ & 10.35 & 4.26 \\
\hline July-89 & $5,582.85$ & 1,590 & 4,091 & 414 & 0 & 14,877 & 273 & 3 & 6 & 390 & 0.70 & 0.01 & $-3,191.43$ & 10.29 & 0.65 \\
\hline
\end{tabular}


[AVWTP, Ashley Valley Water Treatment Plant; $\sigma$, sigma (standard deviation); 一, no data]

\begin{tabular}{|c|c|c|c|c|c|c|c|c|c|c|c|c|c|c|c|}
\hline \multirow{2}{*}{ Month/year } & \multirow{2}{*}{$\begin{array}{l}\text { Reservoir } \\
\text { altitude } \\
\text { (feet) }\end{array}$} & \multirow{2}{*}{$\begin{array}{c}\text { Monthly } \\
\text { Big Brush } \\
\text { Creek } \\
\text { inflow } \\
\text { (acre-feet) }\end{array}$} & \multirow{2}{*}{$\begin{array}{l}\text { Monthly } \\
\text { Red Fleet } \\
\text { Dam out- } \\
\quad \text { flow } \\
\text { (acre-feet) }\end{array}$} & \multicolumn{2}{|c|}{$\begin{array}{c}\text { Monthly pumped } \\
\text { outflows } \\
\text { (acre-feet) }\end{array}$} & \multirow{2}{*}{$\begin{array}{c}\text { Res- } \\
\text { ervoir } \\
\text { storage } \\
\text { (acre- } \\
\text { feet) }\end{array}$} & \multirow{2}{*}{$\begin{array}{c}\text { Monthly } \\
\text { evapora- } \\
\text { tion } \\
\text { (acre- } \\
\text { feet) }\end{array}$} & \multirow{2}{*}{$\begin{array}{c}\text { Monthly } \\
\text { precipita- } \\
\text { tion } \\
\text { (acre- } \\
\text { feet) }\end{array}$} & \multirow{2}{*}{$\begin{array}{c}\text { Monthly } \\
\text { groundwater } \\
\text { recharge/ } \\
\text { discharge } \\
\text { (-) } \\
\text { (acre-feet) }\end{array}$} & \multirow{2}{*}{$\begin{array}{l}\text { Res- } \\
\text { ervoir } \\
\text { surface } \\
\text { area } \\
\text { (acres) }\end{array}$} & \multirow{2}{*}{$\begin{array}{c}\text { Monthly } \\
\text { evapora- } \\
\text { tion rate } \\
\text { (feet) }\end{array}$} & \multirow{2}{*}{$\begin{array}{c}\text { Monthly } \\
\text { precipita- } \\
\text { tion rate } \\
\text { (feet) }\end{array}$} & \multirow{2}{*}{$\begin{array}{l}\text { Monthly } \\
\text { reservoir } \\
\text { storage } \\
\text { change } \\
\text { (acre-feet) }\end{array}$} & \multirow{2}{*}{$\begin{array}{c}\text { Monthly } \\
\text { groundwater } \\
\text { recharge/ } \\
\text { discharge un- } \\
\text { certainty, } 2 \sigma \\
\text { (percent) }\end{array}$} & \multirow{2}{*}{$\begin{array}{c}\text { Monthly } \\
\text { groundwater } \\
\text { recharge/ } \\
\text { discharge un- } \\
\text { certainty, } 2 \sigma \\
\text { (acre-feet) }\end{array}$} \\
\hline & & & & AVWTP & $\begin{array}{c}\text { Irriga- } \\
\text { tion }\end{array}$ & & & & & & & & & & \\
\hline August-89 & $5,576.19$ & 1,250 & 3,051 & 294 & 0 & 12,563 & 187 & 21 & 53 & 355 & 0.53 & 0.06 & $-2,313.38$ & 10.29 & 5.42 \\
\hline September-89 & $5,573.27$ & 936 & 1,528 & 127 & 0 & 11,617 & 112 & 18 & 133 & 325 & 0.35 & 0.05 & -946.71 & 10.35 & 13.78 \\
\hline October-89 & $5,573.13$ & 831 & 717 & 0 & 0 & 11,572 & 59 & 11 & 111 & 320 & 0.18 & 0.04 & -44.41 & 10.42 & 11.62 \\
\hline November-89 & $5,574.36$ & 740 & 238 & 0 & 0 & 11,965 & 15 & 11 & 104 & 323 & 0.05 & 0.03 & 393.20 & 10.18 & 10.60 \\
\hline December-89 & $5,575.91$ & 738 & 171 & 0 & 0 & 12,471 & 1 & 2 & 63 & 330 & 0.00 & 0.01 & 505.41 & 10.02 & 6.31 \\
\hline January-90 & $5,578.04$ & 742 & 41 & 0 & 0 & 13,184 & 3 & 3 & -12 & 340 & 0.01 & 0.01 & 713.21 & 10.04 & 1.20 \\
\hline February-90 & $5,580.11$ & 621 & 0 & 0 & 0 & 13,899 & 3 & 18 & -79 & 351 & 0.01 & 0.05 & 714.64 & 10.15 & 8.02 \\
\hline March-90 & $5,582.32$ & 740 & 0 & 0 & 0 & 14,686 & 48 & 7 & -88 & 363 & 0.13 & 0.02 & 787.37 & 10.35 & 9.14 \\
\hline April-90 & $5,588.44$ & 3,430 & 980 & 0 & 0 & 17,007 & 104 & 38 & 63 & 382 & 0.27 & 0.10 & $2,320.48$ & 10.21 & 6.41 \\
\hline May-90 & $5,594.11$ & 6,170 & 4,011 & 0 & 0 & 19,355 & 162 & 12 & -340 & 417 & 0.39 & 0.03 & $2,348.74$ & 10.14 & 34.43 \\
\hline June-90 & $5,596.19$ & 3,930 & 2,953 & 0 & 0 & 20,259 & 266 & 80 & -113 & 448 & 0.59 & 0.18 & 903.72 & 10.42 & 11.76 \\
\hline July-90 & $5,589.44$ & 1,840 & 4,348 & 0 & 0 & 17,408 & 284 & 5 & 64 & 425 & 0.67 & 0.01 & $-2,850.79$ & 10.31 & 6.55 \\
\hline August-90 & $5,583.39$ & 1,850 & 3,848 & 0 & 0 & 15,077 & 212 & 2 & 124 & 390 & 0.54 & 0.01 & $-2,331.58$ & 10.26 & 12.76 \\
\hline September-90 & $5,581.99$ & 1,440 & 1,468 & 129 & 0 & 14,567 & 144 & 29 & 237 & 370 & 0.39 & 0.08 & -509.78 & 10.47 & 24.83 \\
\hline October-90 & $5,581.94$ & 940 & 875 & 0 & 0 & 14,549 & 59 & 59 & 83 & 366 & 0.16 & 0.16 & -18.01 & 10.60 & 8.82 \\
\hline November-90 & $5,583.61$ & 736 & 129 & 0 & 0 & 15,158 & 14 & 12 & -4 & 370 & 0.04 & 0.03 & 608.87 & 10.17 & 0.39 \\
\hline December-90 & $5,585.27$ & 661 & 99 & 0 & 0 & 15,778 & 0 & 14 & -45 & 380 & 0.00 & 0.04 & 620.41 & 10.10 & 4.58 \\
\hline January-91 & $5,586.67$ & 632 & 123 & 0 & 0 & 16,313 & 0 & 3 & -23 & 389 & 0.00 & 0.01 & 535.33 & 10.03 & 2.31 \\
\hline February-91 & $5,587.93$ & 631 & 111 & 0 & 0 & 16,805 & 10 & 3 & 22 & 396 & 0.03 & 0.01 & 491.50 & 10.11 & 2.17 \\
\hline March-91 & $5,589.72$ & 684 & 44 & 0 & 0 & 17,519 & 37 & 23 & -88 & 405 & 0.09 & 0.06 & 714.43 & 10.40 & 9.16 \\
\hline April-91 & $5,591.9$ & 1,090 & 103 & 0 & 0 & 18,416 & 78 & 46 & 59 & 415 & 0.19 & 0.11 & 896.50 & 10.56 & 6.22 \\
\hline May-91 & $5,603.78$ & 8,070 & 2,977 & 105 & 0 & 23,791 & 169 & 14 & -542 & 448 & 0.38 & 0.03 & $5,374.89$ & 10.11 & 54.83 \\
\hline June-91 & $5,606.58$ & 6,900 & 5,504 & 264 & 0 & 25,184 & 295 & 23 & -533 & 517 & 0.57 & 0.05 & $1,393.27$ & 10.22 & 54.48 \\
\hline July-91 & $5,600.91$ & 2,240 & 4,667 & 0 & 0 & 22,414 & 325 & 8 & 26 & 491 & 0.66 & 0.02 & $-2,770.33$ & 10.33 & 2.72 \\
\hline August-91 & $5,597.62$ & 2,380 & 3,691 & 0 & 0 & 20,898 & 245 & 32 & -8 & 469 & 0.52 & 0.07 & $-1,516.17$ & 10.35 & 0.83 \\
\hline September-91 & $5,596.37$ & 1,930 & 2,471 & 0 & 0 & 20,339 & 148 & 35 & -95 & 450 & 0.33 & 0.08 & -558.84 & 10.36 & 9.88 \\
\hline October-91 & $5,593.79$ & 1,090 & 2,108 & 0 & 0 & 19,215 & 81 & 25 & 50 & 441 & 0.18 & 0.06 & $-1,123.94$ & 10.24 & 5.13 \\
\hline November-91 & $5,594.16$ & 962 & 678 & 0 & 0 & 19,374 & 13 & 10 & 121 & 435 & 0.03 & 0.02 & 158.76 & 10.13 & 12.28 \\
\hline
\end{tabular}


Table 1. Reservoir data, evaporation, and calculated recharge and discharge from Red Fleet Reservoir, Utah, 1980-2015. - Continued

[AVWTP, Ashley Valley Water Treatment Plant; $\sigma$, sigma (standard deviation); - , no data]

\begin{tabular}{|c|c|c|c|c|c|c|c|c|c|c|c|c|c|c|c|}
\hline \multirow{2}{*}{ Month/year } & \multirow{2}{*}{$\begin{array}{l}\text { Reservoir } \\
\text { altitude } \\
\text { (feet) }\end{array}$} & \multirow{2}{*}{$\begin{array}{c}\text { Monthly } \\
\text { Big Brush } \\
\text { Creek } \\
\text { inflow } \\
\text { (acre-feet) }\end{array}$} & \multirow{2}{*}{$\begin{array}{l}\text { Monthly } \\
\text { Red Fleet } \\
\text { Dam out- } \\
\text { flow } \\
\text { (acre-feet) }\end{array}$} & \multicolumn{2}{|c|}{$\begin{array}{l}\text { Monthly pumped } \\
\text { outflows } \\
\text { (acre-feet) }\end{array}$} & \multirow{2}{*}{$\begin{array}{c}\text { Res- } \\
\text { ervoir } \\
\text { storage } \\
\text { (acre- } \\
\text { feet) }\end{array}$} & \multirow{2}{*}{$\begin{array}{c}\text { Monthly } \\
\text { evapora- } \\
\text { tion } \\
\text { (acre- } \\
\text { feet) }\end{array}$} & \multirow{2}{*}{$\begin{array}{l}\text { Monthly } \\
\text { precipita- } \\
\text { tion } \\
\text { (acre- } \\
\text { feet) }\end{array}$} & \multirow{2}{*}{$\begin{array}{c}\text { Monthly } \\
\text { groundwater } \\
\text { recharge/ } \\
\text { discharge } \\
\text { (-) } \\
\text { (acre-feet) }\end{array}$} & \multirow{2}{*}{$\begin{array}{l}\text { Res- } \\
\text { ervoir } \\
\text { surface } \\
\text { area } \\
\text { (acres) }\end{array}$} & \multirow{2}{*}{$\begin{array}{c}\text { Monthly } \\
\text { evapora- } \\
\text { tion rate } \\
\text { (feet) }\end{array}$} & \multirow{2}{*}{$\begin{array}{c}\text { Monthly } \\
\text { precipita- } \\
\text { tion rate } \\
\text { (feet) }\end{array}$} & \multirow{2}{*}{$\begin{array}{c}\text { Monthly } \\
\text { reservoir } \\
\text { storage } \\
\text { change } \\
\text { (acre-feet) }\end{array}$} & \multirow{2}{*}{$\begin{array}{c}\text { Monthly } \\
\text { groundwater } \\
\text { recharge/ } \\
\text { discharge un- } \\
\text { certainty, } 2 \sigma \\
\text { (percent) }\end{array}$} & \multirow{2}{*}{$\begin{array}{c}\text { Monthly } \\
\text { groundwater } \\
\text { recharge/ } \\
\text { discharge un } \\
\text { certainty, } 2 \sigma \\
\text { (acre-feet) }\end{array}$} \\
\hline & & & & AVWTP & $\begin{array}{c}\text { Irriga- } \\
\text { tion }\end{array}$ & & & & & & & & & & \\
\hline December-91 & $5,593.95$ & 801 & 815 & 0 & 0 & 19,283 & 1 & 9 & 85 & 435 & 0.00 & 0.02 & -90.21 & 10.06 & 8.54 \\
\hline January-92 & $5,593.89$ & 760 & 720 & 0 & 0 & 19,258 & 0 & 9 & 74 & 434 & 0.00 & 0.02 & -25.73 & 10.06 & 7.47 \\
\hline February-92 & $5,594.29$ & 610 & 468 & 0 & 0 & 19,430 & 15 & 16 & -29 & 435 & 0.03 & 0.04 & 171.90 & 10.24 & 2.93 \\
\hline March-92 & $5,594.48$ & 793 & 706 & 0 & 0 & 19,512 & 55 & 24 & -26 & 437 & 0.13 & 0.05 & 81.99 & 10.47 & 2.73 \\
\hline April-92 & $5,601.81$ & 4,740 & 1,521 & 0 & 0 & 22,840 & 128 & 6 & -232 & 453 & 0.28 & 0.01 & $3,328.55$ & 10.14 & 23.56 \\
\hline May-92 & $5,608.06$ & 8,670 & 5,937 & 251 & 0 & 25,941 & 211 & 58 & -771 & 514 & 0.41 & 0.11 & $3,100.61$ & 10.15 & 78.22 \\
\hline June-92 & $5,603.92$ & 2,140 & 3,663 & 348 & 0 & 23,859 & 265 & 26 & -29 & 512 & 0.52 & 0.05 & $-2,081.38$ & 10.34 & 2.96 \\
\hline July-92 & $5,600.69$ & 1,930 & 2,922 & 328 & 0 & 22,310 & 267 & 68 & 30 & 482 & 0.55 & 0.14 & $-1,549.00$ & 10.47 & 3.15 \\
\hline August-92 & $5,594.37$ & 1,580 & 3,878 & 358 & 0 & 19,464 & 231 & 28 & -12 & 455 & 0.51 & 0.06 & $-2,846.27$ & 10.29 & 1.27 \\
\hline September-92 & $5,590.61$ & 976 & 2,154 & 204 & 0 & 17,883 & 145 & 22 & 76 & 426 & 0.34 & 0.05 & $-1,580.66$ & 10.33 & 7.81 \\
\hline October-92 & $5,588.89$ & 831 & 1,353 & 0 & 0 & 17,188 & 73 & 34 & 135 & 407 & 0.18 & 0.08 & -695.75 & 10.36 & 14.00 \\
\hline November-92 & $5,589.43$ & 758 & 422 & 0 & 0 & 17,402 & 11 & 21 & 130 & 405 & 0.03 & 0.05 & 214.75 & 10.22 & 13.33 \\
\hline December-92 & $5,590.86$ & 630 & 286 & 0 & 0 & 17,985 & 0 & 24 & -214 & 411 & 0.00 & 0.06 & 582.19 & 10.16 & 21.72 \\
\hline January-93 & $5,591.77$ & 619 & 375 & 0 & 0 & 18,362 & 1 & 27 & -107 & 419 & 0.00 & 0.06 & 377.67 & 10.20 & 10.94 \\
\hline February-93 & $5,592.61$ & 588 & 319 & 0 & 0 & 18,714 & 1 & 34 & -50 & 423 & 0.00 & 0.08 & 351.64 & 10.27 & 5.14 \\
\hline March-93 & $5,594.62$ & 735 & 40 & 0 & 0 & 19,572 & 38 & 68 & -133 & 431 & 0.09 & 0.16 & 858.21 & 10.61 & 14.07 \\
\hline April-93 & $5,594.45$ & 1,750 & 1,745 & 14 & 0 & 19,499 & 81 & 30 & 13 & 440 & 0.18 & 0.07 & -73.51 & 10.30 & 1.37 \\
\hline May-93 & $5,602.62$ & 11,210 & 7,696 & 236 & 0 & 23,228 & 197 & 40 & -608 & 456 & 0.43 & 0.09 & $3,729.50$ & 10.10 & 61.39 \\
\hline June-93 & $5,606.49$ & 8,120 & 8,602 & 220 & 0 & 25,139 & 269 & 33 & $-2,849$ & 510 & 0.53 & 0.07 & $1,910.47$ & 10.16 & 289.37 \\
\hline July-93 & $5,600.13$ & 1,900 & 4,614 & 0 & 0 & 22,048 & 303 & 11 & 84 & 491 & 0.62 & 0.02 & $-3,090.21$ & 10.32 & 8.67 \\
\hline August-93 & $5,597.46$ & 2,280 & 3,606 & 0 & 0 & 20,826 & 240 & 39 & -304 & 467 & 0.51 & 0.08 & $-1,222.79$ & 10.38 & 31.58 \\
\hline September-93 & $5,592.74$ & 1,350 & 3,158 & 0 & 0 & 18,769 & 150 & 18 & 117 & 440 & 0.34 & 0.04 & $-2,056.88$ & 10.25 & 12.00 \\
\hline October-93 & $5,590.5$ & 1,280 & 2,073 & 0 & 0 & 17,838 & 51 & 89 & 176 & 419 & 0.12 & 0.21 & -930.28 & 10.32 & 18.16 \\
\hline November-93 & $5,591.36$ & 930 & 438 & 0 & 0 & 18,192 & 8 & 14 & 144 & 417 & 0.02 & 0.03 & 353.72 & 10.13 & 14.55 \\
\hline December-93 & $5,591.72$ & 821 & 518 & 0 & 0 & 18,341 & 1 & 0 & 153 & 420 & 0.00 & 0.00 & 149.34 & 10.01 & 15.27 \\
\hline January-94 & $5,592.73$ & 803 & 266 & 0 & 0 & 18,764 & 6 & 0 & 109 & 424 & 0.01 & 0.00 & 423.01 & 10.04 & 10.93 \\
\hline February-94 & $5,593.94$ & 682 & 222 & 0 & 0 & 19,279 & 8 & 34 & -29 & 430 & 0.02 & 0.08 & 514.63 & 10.29 & 3.02 \\
\hline March-94 & $5,595.44$ & 869 & 143 & 0 & 0 & 19,929 & 57 & 10 & 29 & 439 & 0.13 & 0.02 & 649.97 & 10.39 & 2.99 \\
\hline
\end{tabular}


[AVWTP, Ashley Valley Water Treatment Plant; $\sigma$, sigma (standard deviation); 一, no data]

\begin{tabular}{|c|c|c|c|c|c|c|c|c|c|c|c|c|c|c|c|}
\hline \multirow{2}{*}{ Month/year } & \multirow{2}{*}{$\begin{array}{l}\text { Reservoir } \\
\text { altitude } \\
\text { (feet) }\end{array}$} & \multirow{2}{*}{$\begin{array}{c}\text { Monthly } \\
\text { Big Brush } \\
\text { Creek } \\
\text { inflow } \\
\text { (acre-feet) }\end{array}$} & \multirow{2}{*}{$\begin{array}{l}\text { Monthly } \\
\text { Red Fleet } \\
\text { Dam out- } \\
\text { flow } \\
\text { (acre-feet) }\end{array}$} & \multicolumn{2}{|c|}{$\begin{array}{c}\text { Monthly pumped } \\
\text { outflows } \\
\text { (acre-feet) }\end{array}$} & \multirow{2}{*}{$\begin{array}{c}\text { Res- } \\
\text { ervoir } \\
\text { storage } \\
\text { (acre- } \\
\text { feet) }\end{array}$} & \multirow{2}{*}{$\begin{array}{c}\text { Monthly } \\
\text { evapora- } \\
\text { tion } \\
\text { (acre- } \\
\text { feet) }\end{array}$} & \multirow{2}{*}{$\begin{array}{l}\text { Monthly } \\
\text { precipita- } \\
\text { tion } \\
\text { (acre- } \\
\text { feet) }\end{array}$} & \multirow{2}{*}{$\begin{array}{c}\text { Monthly } \\
\text { groundwater } \\
\text { recharge/ } \\
\text { discharge } \\
\text { (-) } \\
\text { (acre-feet) }\end{array}$} & \multirow{2}{*}{$\begin{array}{l}\text { Res- } \\
\text { ervoir } \\
\text { surface } \\
\text { area } \\
\text { (acres) }\end{array}$} & \multirow{2}{*}{$\begin{array}{l}\text { Monthly } \\
\text { evapora- } \\
\text { tion rate } \\
\text { (feet) }\end{array}$} & \multirow{2}{*}{$\begin{array}{l}\text { Monthly } \\
\text { precipita- } \\
\text { tion rate } \\
\text { (feet) }\end{array}$} & \multirow{2}{*}{$\begin{array}{l}\text { Monthly } \\
\text { reservoir } \\
\text { storage } \\
\text { change } \\
\text { (acre-feet) }\end{array}$} & \multirow{2}{*}{$\begin{array}{l}\text { Monthly } \\
\text { groundwater } \\
\text { recharge/ } \\
\text { discharge un- } \\
\text { certainty, 2 } \sigma \\
\text { (percent) }\end{array}$} & \multirow{2}{*}{$\begin{array}{l}\text { Monthly } \\
\text { groundwater } \\
\text { recharge/ } \\
\text { discharge un- } \\
\text { certainty, } 2 \sigma \\
\text { (acre-feet) }\end{array}$} \\
\hline & & & & AVWTP & $\begin{array}{l}\text { Irriga- } \\
\text { tion }\end{array}$ & & & & & & & & & & \\
\hline April-94 & $5,600.28$ & 3,840 & 1,379 & 80 & 0 & 22,118 & 107 & 17 & 103 & 452 & 0.24 & 0.04 & $2,189.26$ & 10.16 & 10.47 \\
\hline May-94 & $5,607.11$ & 8,740 & 5,290 & 276 & 0 & 25,453 & 218 & 32 & -347 & 498 & 0.44 & 0.06 & $3,335.11$ & 10.14 & 35.24 \\
\hline June-94 & $5,601.55$ & 2,350 & 4,861 & 350 & 0 & 22,716 & 315 & 9 & -431 & 504 & 0.62 & 0.02 & $-2,737.01$ & 10.30 & 44.38 \\
\hline July-94 & $5,591.27$ & 1,760 & 5,171 & 419 & 52 & 18,155 & 299 & 1 & 382 & 447 & 0.67 & 0.00 & $-4,561.54$ & 10.29 & 39.29 \\
\hline August-94 & $5,581.37$ & 1,800 & 4,439 & 336 & 642 & 14,341 & 218 & 23 & 2 & 391 & 0.56 & 0.06 & $-3,813.54$ & 10.78 & 0.18 \\
\hline September-94 & $5,573.8$ & 962 & 2,420 & 222 & 0 & 11,786 & 120 & 23 & 779 & 341 & 0.35 & 0.07 & $-2,555.71$ & 10.23 & 79.66 \\
\hline October-94 & $5,576.87$ & 1,480 & 674 & 0 & 0 & 12,790 & 44 & 106 & -137 & 329 & 0.13 & 0.32 & $1,004.00$ & 10.45 & 14.27 \\
\hline November-94 & $5,579.81$ & 1,170 & 298 & 0 & 0 & 13,794 & 8 & 33 & -106 & 349 & 0.02 & 0.10 & $1,004.20$ & 10.16 & 10.82 \\
\hline December-94 & $5,582.14$ & 964 & 307 & 0 & 0 & 14,621 & 2 & 8 & -164 & 362 & 0.01 & 0.02 & 827.19 & 10.05 & 16.48 \\
\hline January-95 & $5,584.26$ & 895 & 307 & 0 & 0 & 15,399 & 5 & 14 & -181 & 373 & 0.01 & 0.04 & 777.85 & 10.09 & 18.27 \\
\hline February-95 & $5,586.28$ & 817 & 278 & 0 & 0 & 16,163 & 26 & 30 & -221 & 384 & 0.07 & 0.08 & 764.38 & 10.29 & 22.71 \\
\hline March-95 & $5,588.88$ & 1,220 & 307 & 0 & 0 & 17,182 & 44 & 8 & -141 & 397 & 0.11 & 0.02 & $1,018.53$ & 10.20 & 14.43 \\
\hline April-95 & $5,593.63$ & 2,850 & 1,115 & 83 & 0 & 19,149 & 78 & 39 & -354 & 420 & 0.19 & 0.09 & $1,966.86$ & 10.19 & 36.05 \\
\hline May-95 & $5,603.25$ & 10,090 & 5,970 & 127 & 0 & 23,533 & 131 & 107 & -415 & 459 & 0.29 & 0.23 & $4,384.03$ & 10.11 & 41.97 \\
\hline June-95 & $5,608.21$ & 17,040 & 14,652 & 242 & 0 & 26,018 & 260 & 51 & -548 & 516 & 0.50 & 0.10 & $2,485.50$ & 10.09 & 55.31 \\
\hline July-95 & $5,605.72$ & 5,530 & 7,918 & 36 & 0 & 24,751 & 328 & 38 & $-1,447$ & 518 & 0.63 & 0.07 & $-1,267.29$ & 10.24 & 148.16 \\
\hline August-95 & $5,599.97$ & 2,200 & 5,236 & 0 & 0 & 21,974 & 296 & 29 & -526 & 490 & 0.60 & 0.06 & $-2,777.05$ & 10.31 & 54.17 \\
\hline September-95 & $5,596.02$ & 1,610 & 3,253 & 0 & 0 & 20,184 & 167 & 24 & 4 & 454 & 0.37 & 0.05 & $-1,789.87$ & 10.28 & 0.43 \\
\hline October-95 & $5,596.68$ & 1,410 & 996 & 0 & 0 & 20,476 & 72 & 6 & 56 & 449 & 0.16 & 0.01 & 292.51 & 10.28 & 5.78 \\
\hline November-95 & $5,596.28$ & 930 & 1,174 & 0 & 0 & 20,299 & 26 & 3 & -89 & 449 & 0.06 & 0.01 & -177.59 & 10.13 & 8.99 \\
\hline December-95 & $5,596.16$ & 927 & 984 & 0 & 0 & 20,246 & 9 & 2 & -10 & 448 & 0.02 & 0.01 & -53.09 & 10.05 & 1.00 \\
\hline January-96 & $5,597.14$ & 845 & 504 & 0 & 0 & 20,682 & 3 & 27 & -71 & 449 & 0.01 & 0.06 & 436.10 & 10.16 & 7.26 \\
\hline February-96 & $5,598.2$ & 847 & 288 & 0 & 0 & 21,160 & 14 & 19 & 87 & 456 & 0.03 & 0.04 & 478.21 & 10.20 & 8.87 \\
\hline March-96 & $5,599.29$ & 956 & 307 & 0 & 0 & 21,659 & 47 & 19 & 121 & 463 & 0.10 & 0.04 & 498.85 & 10.36 & 12.57 \\
\hline April-96 & $5,600.51$ & 1,930 & 1,099 & 15 & 0 & 22,226 & 90 & 17 & 176 & 471 & 0.19 & 0.04 & 566.94 & 10.29 & 18.10 \\
\hline May-96 & $5,605.24$ & 6,450 & 4,655 & 279 & 0 & 24,511 & 151 & 52 & -868 & 485 & 0.31 & 0.11 & $2,285.23$ & 10.15 & 88.10 \\
\hline June-96 & $5,606.07$ & 4,720 & 4,403 & 361 & 0 & 24,927 & 272 & 29 & -703 & 517 & 0.53 & 0.06 & 415.49 & 10.30 & 72.38 \\
\hline July-96 & $5,600.98$ & 2,390 & 4,681 & 317 & 0 & 22,447 & 353 & 63 & -419 & 493 & 0.72 & 0.13 & $-2,479.87$ & 10.40 & 43.56 \\
\hline
\end{tabular}


Table 1. Reservoir data, evaporation, and calculated recharge and discharge from Red Fleet Reservoir, Utah, 1980-2015. - Continued

[AVWTP, Ashley Valley Water Treatment Plant; $\sigma$, sigma (standard deviation); - , no data]

\begin{tabular}{|c|c|c|c|c|c|c|c|c|c|c|c|c|c|c|c|}
\hline \multirow{2}{*}{ Month/year } & \multirow{2}{*}{$\begin{array}{c}\text { Reservoir } \\
\text { altitude } \\
\text { (feet) }\end{array}$} & \multirow{2}{*}{$\begin{array}{c}\text { Monthly } \\
\text { Big Brush } \\
\text { Creek } \\
\text { inflow } \\
\text { (acre-feet) }\end{array}$} & \multirow{2}{*}{$\begin{array}{l}\text { Monthly } \\
\text { Red Fleet } \\
\text { Dam out- } \\
\text { flow } \\
\text { (acre-feet) }\end{array}$} & \multicolumn{2}{|c|}{$\begin{array}{l}\text { Monthly pumped } \\
\text { outflows } \\
\text { (acre-feet) }\end{array}$} & \multirow{2}{*}{ 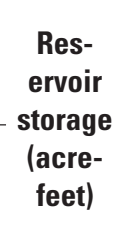 } & \multirow{2}{*}{$\begin{array}{c}\text { Monthly } \\
\text { evapora- } \\
\text { tion } \\
\text { (acre- } \\
\text { feet) }\end{array}$} & \multirow{2}{*}{$\begin{array}{l}\text { Monthly } \\
\text { precipita- } \\
\text { tion } \\
\text { (acre- } \\
\text { feet) }\end{array}$} & \multirow{2}{*}{$\begin{array}{c}\text { Monthly } \\
\text { groundwater } \\
\text { recharge/ } \\
\text { discharge } \\
\text { (-) } \\
\text { (acre-feet) }\end{array}$} & \multirow{2}{*}{$\begin{array}{l}\text { Res- } \\
\text { ervoir } \\
\text { surface } \\
\text { area } \\
\text { (acres) }\end{array}$} & \multirow{2}{*}{$\begin{array}{c}\text { Monthly } \\
\text { evapora- } \\
\text { tion rate } \\
\text { (feet) }\end{array}$} & \multirow{2}{*}{$\begin{array}{c}\text { Monthly } \\
\text { precipita- } \\
\text { tion rate } \\
\text { (feet) }\end{array}$} & \multirow{2}{*}{$\begin{array}{l}\text { Monthly } \\
\text { reservoir } \\
\text { storage } \\
\text { change } \\
\text { (acre-feet) }\end{array}$} & \multirow{2}{*}{$\begin{array}{c}\text { Monthly } \\
\text { groundwater } \\
\text { recharge/ } \\
\text { discharge un- } \\
\text { certainty, } 2 \sigma \\
\text { (percent) }\end{array}$} & \multirow{2}{*}{$\begin{array}{c}\text { Monthly } \\
\text { groundwater } \\
\text { recharge/ } \\
\text { discharge un- } \\
\text { certainty, } 2 \sigma \\
\text { (acre-feet) }\end{array}$} \\
\hline & & & & AVWTP & $\begin{array}{l}\text { Irriga- } \\
\text { tion }\end{array}$ & & & & & & & & & & \\
\hline August-96 & $5,593.57$ & 1,730 & 4,794 & 106 & 116 & 19,121 & 263 & 1 & -223 & 454 & 0.58 & 0.00 & $-3,325.95$ & 10.37 & 23.10 \\
\hline September-96 & $5,588.89$ & 1,050 & 2,267 & 224 & 340 & 17,188 & 109 & 35 & 78 & 413 & 0.26 & 0.09 & $-1,933.17$ & 10.81 & 8.42 \\
\hline October-96 & $5,589.59$ & 974 & 629 & 0 & 0 & 17,467 & 63 & 34 & 37 & 407 & 0.15 & 0.08 & 279.27 & 10.49 & 3.92 \\
\hline November-96 & $5,590.37$ & 845 & 595 & 0 & 0 & 17,784 & 13 & 25 & -55 & 411 & 0.03 & 0.06 & 316.77 & 10.22 & 5.60 \\
\hline December-96 & $5,591.16$ & 906 & 615 & 0 & 0 & 18,109 & 3 & 18 & -20 & 415 & 0.01 & 0.04 & 325.79 & 10.11 & 1.99 \\
\hline January-97 & $5,592.1$ & 841 & 615 & 0 & 0 & 18,500 & 1 & 46 & -120 & 421 & 0.00 & 0.11 & 390.44 & 10.25 & 12.32 \\
\hline February-97 & $5,592.79$ & 774 & 555 & 0 & 0 & 18,790 & 1 & 25 & -48 & 425 & 0.00 & 0.06 & 289.87 & 10.16 & 4.86 \\
\hline March-97 & $5,593.23$ & 1,090 & 1,531 & 0 & 0 & 18,976 & 46 & 2 & -672 & 432 & 0.11 & 0.00 & 186.30 & 10.17 & 68.28 \\
\hline April-97 & $5,590.89$ & 1,960 & 3,011 & 42 & 0 & 17,998 & 56 & 42 & -129 & 420 & 0.13 & 0.10 & -977.81 & 10.16 & 13.09 \\
\hline May-97 & $5,600.43$ & 10,820 & 8,467 & 324 & 0 & 22,188 & 135 & 22 & $-2,274$ & 437 & 0.31 & 0.05 & $4,190.18$ & 10.07 & 228.90 \\
\hline June-97 & $5,603.6$ & 5,940 & 4,701 & 358 & 0 & 23,703 & 237 & 40 & -831 & 498 & 0.48 & 0.08 & $1,514.51$ & 10.22 & 84.85 \\
\hline July-97 & $5,596.27$ & 2,340 & 5,415 & 217 & 90 & 20,294 & 227 & 13 & -187 & 468 & 0.48 & 0.03 & $-3,408.50$ & 10.28 & 19.19 \\
\hline August-97 & $5,593.69$ & 2,000 & 3,239 & 172 & 10 & 19,172 & 174 & 89 & -384 & 442 & 0.39 & 0.20 & $-1,122.41$ & 10.40 & 39.96 \\
\hline September-97 & $5,592.85$ & 2,090 & 2,636 & 0 & 0 & 18,815 & 111 & 101 & -199 & 423 & 0.26 & 0.24 & -356.95 & 10.40 & 20.70 \\
\hline October-97 & $5,596.49$ & 2,050 & 337 & 0 & 0 & 20,392 & 65 & 25 & 96 & 439 & 0.15 & 0.06 & $1,576.90$ & 10.22 & 9.77 \\
\hline November-97 & $5,597.45$ & 1,700 & 1,369 & 0 & 0 & 20,821 & 13 & 9 & -102 & 455 & 0.03 & 0.02 & 429.05 & 10.06 & 10.24 \\
\hline December-97 & $5,596.55$ & 1,320 & 1,815 & 0 & 0 & 20,419 & 1 & 25 & -68 & 452 & 0.00 & 0.06 & -402.39 & 10.07 & 6.87 \\
\hline January-98 & $5,596.63$ & 1,120 & 1,230 & 0 & 0 & 20,454 & 6 & 13 & -138 & 450 & 0.01 & 0.03 & 35.57 & 10.08 & 13.96 \\
\hline February-98 & $5,596.55$ & 946 & 1,111 & 0 & 0 & 20,419 & 14 & 44 & -99 & 450 & 0.03 & 0.10 & -35.57 & 10.27 & 10.18 \\
\hline March-98 & $5,596.84$ & 968 & 1,230 & 0 & 0 & 20,548 & 51 & 29 & -413 & 450 & 0.11 & 0.06 & 129.13 & 10.33 & 42.65 \\
\hline April-98 & $5,597.79$ & 2,300 & 1,974 & 47 & 0 & 20,974 & 107 & 5 & -249 & 453 & 0.24 & 0.01 & 426.55 & 10.23 & 25.51 \\
\hline May-98 & $5,608.28$ & 18,200 & 11,712 & 270 & 0 & 26,054 & 203 & 4 & 939 & 496 & 0.41 & 0.01 & $5,080.04$ & 10.06 & 94.46 \\
\hline June-98 & $5,608.12$ & 13,840 & 13,932 & 0 & 0 & 25,972 & 270 & 54 & -225 & 524 & 0.52 & 0.10 & -82.70 & 10.12 & 22.80 \\
\hline July-98 & $5,605.21$ & 3,550 & 5,576 & 0 & 0 & 24,496 & 370 & 76 & -844 & 516 & 0.72 & 0.15 & $-1,475.48$ & 10.40 & 87.80 \\
\hline August-98 & $5,600.77$ & 2,660 & 4,931 & 0 & 0 & 22,348 & 287 & 41 & -369 & 492 & 0.58 & 0.08 & $-2,148.29$ & 10.33 & 38.13 \\
\hline September-98 & $5,595.74$ & 1,750 & 3,808 & 0 & 0 & 20,061 & 186 & 17 & 60 & 457 & 0.41 & 0.04 & $-2,287.25$ & 10.25 & 6.16 \\
\hline October-98 & $5,594.54$ & 1,330 & 1,825 & 0 & 0 & 19,537 & 78 & 63 & 13 & 439 & 0.18 & 0.14 & -523.18 & 10.37 & 1.33 \\
\hline November-98 & $5,594.81$ & 1,170 & 1,071 & 0 & 0 & 19,654 & 22 & 30 & -10 & 439 & 0.05 & 0.07 & 116.97 & 10.22 & 1.02 \\
\hline
\end{tabular}


[AVWTP, Ashley Valley Water Treatment Plant; $\sigma$, sigma (standard deviation); 一, no data]

\begin{tabular}{|c|c|c|c|c|c|c|c|c|c|c|c|c|c|c|c|}
\hline \multirow{2}{*}{ Month/year } & \multirow{2}{*}{$\begin{array}{l}\text { Reservoir } \\
\text { altitude } \\
\text { (feet) }\end{array}$} & \multirow{2}{*}{$\begin{array}{c}\text { Monthly } \\
\text { Big Brush } \\
\text { Creek } \\
\text { inflow } \\
\text { (acre-feet) }\end{array}$} & \multirow{2}{*}{$\begin{array}{l}\text { Monthly } \\
\text { Red Fleet } \\
\text { Dam out- } \\
\text { flow } \\
\text { (acre-feet) }\end{array}$} & \multicolumn{2}{|c|}{$\begin{array}{c}\text { Monthly pumped } \\
\text { outflows } \\
\text { (acre-feet) }\end{array}$} & \multirow{2}{*}{$\begin{array}{c}\text { Res- } \\
\text { ervoir } \\
\text { storage } \\
\text { (acre- } \\
\text { feet) }\end{array}$} & \multirow{2}{*}{$\begin{array}{c}\text { Monthly } \\
\text { evapora- } \\
\text { tion } \\
\text { (acre- } \\
\text { feet) }\end{array}$} & \multirow{2}{*}{$\begin{array}{l}\text { Monthly } \\
\text { precipita- } \\
\text { tion } \\
\text { (acre- } \\
\text { feet) }\end{array}$} & \multirow{2}{*}{$\begin{array}{c}\text { Monthly } \\
\text { groundwater } \\
\text { recharge/ } \\
\text { discharge } \\
\text { (-) } \\
\text { (acre-feet) }\end{array}$} & \multirow{2}{*}{$\begin{array}{l}\text { Res- } \\
\text { ervoir } \\
\text { surface } \\
\text { area } \\
\text { (acres) }\end{array}$} & \multirow{2}{*}{$\begin{array}{l}\text { Monthly } \\
\text { evapora- } \\
\text { tion rate } \\
\text { (feet) }\end{array}$} & \multirow{2}{*}{$\begin{array}{l}\text { Monthly } \\
\text { precipita- } \\
\text { tion rate } \\
\text { (feet) }\end{array}$} & \multirow{2}{*}{$\begin{array}{l}\text { Monthly } \\
\text { reservoir } \\
\text { storage } \\
\text { change } \\
\text { (acre-feet) }\end{array}$} & \multirow{2}{*}{$\begin{array}{l}\text { Monthly } \\
\text { groundwater } \\
\text { recharge/ } \\
\text { discharge un- } \\
\text { certainty, } 2 \sigma \\
\text { (percent) }\end{array}$} & \multirow{2}{*}{$\begin{array}{l}\text { Monthly } \\
\text { groundwater } \\
\text { recharge/ } \\
\text { discharge un- } \\
\text { certainty, } 2 \sigma \\
\text { (acre-feet) }\end{array}$} \\
\hline & & & & AVWTP & $\begin{array}{l}\text { Irriga- } \\
\text { tion }\end{array}$ & & & & & & & & & & \\
\hline December-98 & $5,594.47$ & 1,170 & 1,230 & 0 & 0 & 19,507 & 4 & 9 & 92 & 438 & 0.01 & 0.02 & -147.22 & 10.05 & 9.30 \\
\hline January-99 & $5,595.17$ & 1,030 & 635 & 0 & 0 & 19,811 & 4 & 29 & 117 & 438 & 0.01 & 0.07 & 303.86 & 10.16 & 11.88 \\
\hline February-99 & $5,596.48$ & 781 & 278 & 0 & 0 & 20,388 & 18 & 21 & -70 & 446 & 0.04 & 0.05 & 576.48 & 10.23 & 7.17 \\
\hline March-99 & $5,597.81$ & 930 & 307 & 0 & 0 & 20,983 & 74 & 0 & -47 & 453 & 0.16 & 0.00 & 595.81 & 10.39 & 4.86 \\
\hline April-99 & $5,598.62$ & 1,570 & 1,309 & 34 & 0 & 21,351 & 86 & 68 & -160 & 461 & 0.19 & 0.15 & 368.10 & 10.45 & 16.73 \\
\hline May-99 & $5,603.43$ & 9,260 & 7,220 & 226 & 0 & 23,620 & 179 & 61 & -573 & 470 & 0.38 & 0.13 & $2,268.68$ & 10.12 & 57.96 \\
\hline June-99 & $5,607.73$ & 10,350 & 8,975 & 206 & 0 & 25,771 & 281 & 13 & $-1,250$ & 519 & 0.54 & 0.02 & $2,150.64$ & 10.13 & 126.69 \\
\hline July-99 & $5,603.66$ & 2,510 & 4,947 & 0 & 0 & 23,732 & 336 & 29 & -706 & 506 & 0.67 & 0.06 & $-2,038.56$ & 10.37 & 73.21 \\
\hline August-99 & $5,600.02$ & 2,480 & 4,403 & 0 & 0 & 21,997 & 271 & 84 & -376 & 482 & 0.56 & 0.17 & $-1,735.13$ & 10.40 & 39.04 \\
\hline September-99 & $5,598.97$ & 1,930 & 2,430 & 0 & 0 & 21,512 & 163 & 43 & -135 & 470 & 0.35 & 0.09 & -485.34 & 10.41 & 14.07 \\
\hline October-99 & $5,597.18$ & 1,340 & 2,043 & 0 & 0 & 20,700 & 98 & 2 & 13 & 458 & 0.21 & 0.01 & -811.94 & 10.23 & 1.38 \\
\hline November-99 & $5,596.82$ & 1,070 & 1,190 & 0 & 0 & 20,539 & 33 & 4 & 11 & 453 & 0.07 & 0.01 & -160.96 & 10.15 & 1.16 \\
\hline December-99 & $5,596.67$ & 972 & 1,123 & 0 & 0 & 20,472 & 2 & 4 & -82 & 451 & 0.01 & 0.01 & -66.84 & 10.03 & 8.26 \\
\hline January-00 & $5,596.51$ & 879 & 1,097 & 0 & 0 & 20,401 & 11 & 18 & -140 & 450 & 0.03 & 0.04 & -71.14 & 10.14 & 14.24 \\
\hline February-00 & $5,596.53$ & 783 & 978 & 0 & 0 & 20,410 & 19 & 37 & -185 & 449 & 0.04 & 0.08 & 8.88 & 10.31 & 19.08 \\
\hline March-00 & $5,596.22$ & 807 & 1,045 & 0 & 0 & 20,272 & 59 & 21 & -138 & 449 & 0.13 & 0.05 & -137.44 & 10.39 & 14.35 \\
\hline April-00 & $5,598.05$ & 3,370 & 2,529 & 66 & 0 & 21,092 & 145 & 22 & -167 & 448 & 0.32 & 0.05 & 819.69 & 10.24 & 17.14 \\
\hline May-00 & $5,608.24$ & 11,820 & 6,893 & 283 & 0 & 26,034 & 251 & 20 & -528 & 506 & 0.50 & 0.04 & $4,941.66$ & 10.11 & 53.37 \\
\hline June-00 & $5,604.8$ & 2,720 & 4,572 & 205 & 0 & 24,293 & 317 & 8 & -624 & 515 & 0.61 & 0.02 & $-1,741.04$ & 10.34 & 64.56 \\
\hline July-00 & $5,601.34$ & 2,170 & 3,898 & 0 & 0 & 22,617 & 356 & 11 & -397 & 492 & 0.72 & 0.02 & $-1,675.80$ & 10.45 & 41.50 \\
\hline August-00 & $5,593.21$ & 1,140 & 4,443 & 0 & 452 & 18,968 & 273 & 19 & -360 & 455 & 0.60 & 0.04 & $-3,649.26$ & 10.74 & 38.65 \\
\hline September- 00 & $5,588.4$ & 839 & 2,483 & 0 & 496 & 16,993 & 146 & 56 & -256 & 411 & 0.36 & 0.14 & $-1,975.05$ & 11.16 & 28.53 \\
\hline October-00 & $5,589.49$ & 1,020 & 666 & 0 & 0 & 17,427 & 67 & 56 & -91 & 404 & 0.16 & 0.14 & 434.04 & 10.55 & 9.62 \\
\hline November-00 & $5,591.06$ & 954 & 298 & 0 & 0 & 18,067 & 7 & 11 & 20 & 413 & 0.02 & 0.03 & 640.45 & 10.10 & 2.06 \\
\hline December-00 & $5,592.37$ & 857 & 307 & 0 & 0 & 18,613 & 2 & 2 & 4 & 421 & 0.00 & 0.00 & 545.99 & 10.02 & 0.36 \\
\hline January-01 & $5,593.56$ & 809 & 307 & 0 & 0 & 19,117 & 1 & 16 & 13 & 428 & 0.00 & 0.04 & 503.55 & 10.11 & 1.36 \\
\hline February-01 & $5,594.6$ & 670 & 278 & 0 & 0 & 19,563 & 15 & 25 & -45 & 435 & 0.04 & 0.06 & 446.89 & 10.28 & 4.66 \\
\hline March-01 & $5,595.61$ & 762 & 307 & 0 & 0 & 20,004 & 73 & 17 & -42 & 441 & 0.17 & 0.04 & 440.13 & 10.56 & 4.42 \\
\hline
\end{tabular}


Table 1. Reservoir data, evaporation, and calculated recharge and discharge from Red Fleet Reservoir, Utah, 1980-2015. - Continued

[AVWTP, Ashley Valley Water Treatment Plant; $\sigma$, sigma (standard deviation); - , no data]

\begin{tabular}{|c|c|c|c|c|c|c|c|c|c|c|c|c|c|c|c|}
\hline \multirow[t]{2}{*}{ Month/year } & \multirow{2}{*}{$\begin{array}{l}\text { Reservoir } \\
\text { altitude } \\
\text { (feet) }\end{array}$} & \multirow{2}{*}{$\begin{array}{c}\text { Monthly } \\
\text { Big Brush } \\
\text { Creek } \\
\text { inflow } \\
\text { (acre-feet) }\end{array}$} & \multirow{2}{*}{$\begin{array}{l}\text { Monthly } \\
\text { Red Fleet } \\
\text { Dam out- } \\
\text { flow } \\
\text { (acre-feet) }\end{array}$} & \multicolumn{2}{|c|}{$\begin{array}{c}\text { Monthly pumped } \\
\text { outflows } \\
\text { (acre-feet) }\end{array}$} & \multirow{2}{*}{$\begin{array}{c}\text { Res- } \\
\text { ervoir } \\
\text { storage } \\
\text { (acre- } \\
\text { feet) }\end{array}$} & \multirow{2}{*}{$\begin{array}{c}\text { Monthly } \\
\text { evapora- } \\
\text { tion } \\
\text { (acre- } \\
\text { feet) }\end{array}$} & \multirow{2}{*}{$\begin{array}{c}\text { Monthly } \\
\text { precipita- } \\
\text { tion } \\
\text { (acre- } \\
\text { feet) }\end{array}$} & \multirow{2}{*}{$\begin{array}{c}\text { Monthly } \\
\text { groundwater } \\
\text { recharge/ } \\
\text { discharge } \\
\text { (-) } \\
\text { (acre-feet) }\end{array}$} & \multirow{2}{*}{$\begin{array}{l}\text { Res- } \\
\text { ervoir } \\
\text { surface } \\
\text { area } \\
\text { (acres) }\end{array}$} & \multirow{2}{*}{$\begin{array}{c}\text { Monthly } \\
\text { evapora- } \\
\text { tion rate } \\
\text { (feet) }\end{array}$} & \multirow{2}{*}{$\begin{array}{c}\text { Monthly } \\
\text { precipita- } \\
\text { tion rate } \\
\text { (feet) }\end{array}$} & \multirow{2}{*}{$\begin{array}{c}\text { Monthly } \\
\text { reservoir } \\
\text { storage } \\
\text { change } \\
\text { (acre-feet) }\end{array}$} & \multirow{2}{*}{$\begin{array}{c}\text { Monthly } \\
\text { groundwater } \\
\text { recharge/ } \\
\text { discharge un- } \\
\text { certainty, } 2 \sigma \\
\text { (percent) }\end{array}$} & \multirow{2}{*}{$\begin{array}{c}\text { Monthly } \\
\text { groundwater } \\
\text { recharge/ } \\
\text { discharge un- } \\
\text { certainty, } 2 \sigma \\
\text { (acre-feet) }\end{array}$} \\
\hline & & & & AVWTP & $\begin{array}{l}\text { Irriga- } \\
\text { tion }\end{array}$ & & & & & & & & & & \\
\hline April-01 & $5,598.95$ & 3,190 & 803 & 60 & 0 & 21,503 & 120 & 46 & 753 & 453 & 0.27 & 0.10 & $1,499.01$ & 10.29 & 77.48 \\
\hline May-01 & $5,608.26$ & 14,640 & 10,602 & 279 & 0 & 26,044 & 228 & 9 & $-1,001$ & 506 & 0.45 & 0.02 & $4,541.45$ & 10.08 & 100.87 \\
\hline June-01 & $5,608.02$ & 3,700 & 5,135 & 78 & 72 & 25,920 & 338 & 18 & $-1,781$ & 521 & 0.65 & 0.03 & -123.96 & 10.45 & 186.15 \\
\hline July-01 & $5,600.73$ & 2,120 & 4,403 & 0 & 256 & 22,329 & 349 & 40 & 743 & 490 & 0.71 & 0.08 & $-3,590.96$ & 10.60 & 78.75 \\
\hline August-01 & $5,599.97$ & 1,720 & 4,197 & 0 & 350 & 21,974 & 270 & 38 & $-2,704$ & 455 & 0.59 & 0.08 & -355.28 & 10.95 & 296.01 \\
\hline September- 01 & $5,588.65$ & 1,010 & 3,302 & 0 & 540 & 17,092 & 176 & 4 & 1,877 & 421 & 0.42 & 0.01 & $-4,881.93$ & 10.73 & 201.37 \\
\hline October-01 & $5,586.95$ & 962 & 1,309 & 0 & 0 & 16,422 & 83 & 29 & 269 & 393 & 0.21 & 0.07 & -669.97 & 10.36 & 27.85 \\
\hline November-01 & $5,588.53$ & 908 & 179 & 0 & 0 & 17,042 & 27 & 9 & 91 & 398 & 0.07 & 0.02 & 620.41 & 10.20 & 9.25 \\
\hline December-01 & $5,590.13$ & 918 & 184 & 0 & 0 & 17,686 & 0 & 1 & 91 & 407 & 0.00 & 0.00 & 643.46 & 10.00 & 9.08 \\
\hline January-02 & $5,591.52$ & 861 & 184 & 0 & 0 & 18,258 & 1 & 0 & 103 & 416 & 0.00 & 0.00 & 572.58 & 10.01 & 10.32 \\
\hline February-02 & $5,592.68$ & 724 & 167 & 0 & 0 & 18,743 & 7 & 0 & 66 & 423 & 0.02 & 0.00 & 484.99 & 10.05 & 6.61 \\
\hline March-02 & $5,594.06$ & 783 & 184 & 0 & 0 & 19,331 & 51 & 20 & -19 & 431 & 0.12 & 0.05 & 587.22 & 10.44 & 2.01 \\
\hline April-02 & $5,593.97$ & 1,440 & 1,402 & 85 & 0 & 19,292 & 135 & 0 & -144 & 437 & 0.31 & 0.00 & -38.64 & 10.44 & 15.01 \\
\hline May-02 & $5,589.99$ & 2,330 & 3,735 & 259 & 240 & 17,631 & 204 & 0 & -446 & 426 & 0.48 & 0.00 & $-1,661.32$ & 10.53 & 46.97 \\
\hline June-02 & $5,582.3$ & 1,440 & 3,178 & 263 & 827 & 14,679 & 266 & 0 & -142 & 390 & 0.68 & 0.00 & $-2,951.76$ & 11.22 & 15.95 \\
\hline July-02 & $5,575.19$ & 887 & 2,698 & 0 & 430 & 12,235 & 265 & 12 & -50 & 345 & 0.77 & 0.03 & $-2,444.13$ & 11.05 & 5.54 \\
\hline August-02 & $5,566.74$ & 739 & 2,793 & 0 & 447 & 9,634 & 180 & 0 & -81 & 308 & 0.59 & 0.00 & $-2,600.28$ & 10.93 & 8.84 \\
\hline September-02 & $5,565.15$ & 776 & 700 & 0 & 274 & 9,199 & 93 & 53 & 198 & 281 & 0.33 & 0.19 & -435.79 & 11.80 & 23.33 \\
\hline October- 02 & $5,565.23$ & 797 & 492 & 0 & 0 & 9,221 & 46 & 21 & 257 & 281 & 0.16 & 0.07 & 22.35 & 10.48 & 26.97 \\
\hline November- 02 & $5,567.16$ & 712 & 119 & 0 & 0 & 9,769 & 10 & 22 & 57 & 286 & 0.03 & 0.08 & 548.38 & 10.23 & 5.85 \\
\hline December-02 & $5,569.53$ & 704 & 123 & 0 & 0 & 10,468 & 0 & 7 & -110 & 297 & 0.00 & 0.03 & 698.15 & 10.05 & 11.03 \\
\hline January-03 & $5,571.62$ & 682 & 123 & 0 & 0 & 11,106 & 1 & 12 & -69 & 307 & 0.00 & 0.04 & 638.80 & 10.09 & 6.95 \\
\hline February- 03 & $5,573.37$ & 583 & 111 & 0 & 0 & 11,648 & 7 & 25 & -52 & 317 & 0.02 & 0.08 & 542.14 & 10.25 & 5.33 \\
\hline March-03 & $5,575.28$ & 660 & 123 & 0 & 0 & 12,264 & 46 & 22 & -103 & 327 & 0.14 & 0.07 & 615.65 & 10.46 & 10.78 \\
\hline April-03 & $5,578.79$ & 2,540 & 1,021 & 72 & 0 & 13,441 & 88 & 5 & 187 & 338 & 0.26 & 0.01 & $1,176.42$ & 10.19 & 19.01 \\
\hline May-03 & $5,591.77$ & 8,480 & 3,289 & 210 & 28 & 18,362 & 160 & 61 & -66 & 374 & 0.43 & 0.16 & $4,921.10$ & 10.15 & 6.72 \\
\hline June-03 & $5,593.62$ & 3,300 & 3,414 & 145 & 0 & 19,142 & 241 & 38 & $-1,242$ & 431 & 0.56 & 0.09 & 780.50 & 10.35 & 128.60 \\
\hline July-03 & $5,588.86$ & 2,390 & 3,993 & 0 & 368 & 17,176 & 329 & 0 & -333 & 419 & 0.78 & 0.00 & $-1,966.49$ & 10.77 & 35.87 \\
\hline
\end{tabular}


[AVWTP, Ashley Valley Water Treatment Plant; $\sigma$, sigma (standard deviation); - , no data]

\begin{tabular}{|c|c|c|c|c|c|c|c|c|c|c|c|c|c|c|c|}
\hline \multirow{2}{*}{ Month/year } & \multirow{2}{*}{$\begin{array}{l}\text { Reservoir } \\
\text { altitude } \\
\text { (feet) }\end{array}$} & \multirow{2}{*}{$\begin{array}{c}\text { Monthly } \\
\text { Big Brush } \\
\text { Creek } \\
\text { inflow } \\
\text { (acre-feet) }\end{array}$} & \multirow{2}{*}{$\begin{array}{l}\text { Monthly } \\
\text { Red Fleet } \\
\text { Dam out- } \\
\quad \text { flow } \\
\text { (acre-feet) }\end{array}$} & \multicolumn{2}{|c|}{$\begin{array}{c}\text { Monthly pumped } \\
\text { outflows } \\
\text { (acre-feet) }\end{array}$} & \multirow{2}{*}{$\begin{array}{c}\text { Res- } \\
\text { ervoir } \\
\text { storage } \\
\text { (acre- } \\
\text { feet) }\end{array}$} & \multirow{2}{*}{$\begin{array}{c}\text { Monthly } \\
\text { evapora- } \\
\text { tion } \\
\text { (acre- } \\
\text { feet) }\end{array}$} & \multirow{2}{*}{$\begin{array}{c}\text { Monthly } \\
\text { precipita- } \\
\text { tion } \\
\text { (acre- } \\
\text { feet) }\end{array}$} & \multirow{2}{*}{$\begin{array}{c}\text { Monthly } \\
\text { groundwater } \\
\text { recharge/ } \\
\text { discharge } \\
\text { (-) } \\
\text { (acre-feet) }\end{array}$} & \multirow{2}{*}{$\begin{array}{l}\text { Res- } \\
\text { ervoir } \\
\text { surface } \\
\text { area } \\
\text { (acres) }\end{array}$} & \multirow{2}{*}{$\begin{array}{l}\text { Monthly } \\
\text { evapora- } \\
\text { tion rate } \\
\text { (feet) }\end{array}$} & \multirow{2}{*}{$\begin{array}{c}\text { Monthly } \\
\text { precipita- } \\
\text { tion rate } \\
\text { (feet) }\end{array}$} & \multirow{2}{*}{$\begin{array}{l}\text { Monthly } \\
\text { reservoir } \\
\text { storage } \\
\text { change } \\
\text { (acre-feet) }\end{array}$} & \multirow{2}{*}{$\begin{array}{c}\text { Monthly } \\
\text { groundwater } \\
\text { recharge/ } \\
\text { discharge un- } \\
\text { certainty, } 2 \sigma \\
\text { (percent) }\end{array}$} & \multirow{2}{*}{$\begin{array}{c}\text { Monthly } \\
\text { groundwater } \\
\text { recharge/ } \\
\text { discharge un- } \\
\text { certainty, } 2 \sigma \\
\text { (acre-feet) }\end{array}$} \\
\hline & & & & AVWTP & $\begin{array}{c}\text { Irriga- } \\
\text { tion }\end{array}$ & & & & & & & & & & \\
\hline August-03 & $5,581.33$ & 1,260 & 3,535 & 0 & 434 & 14,330 & 239 & 10 & -92 & 384 & 0.62 & 0.03 & $-2,845.42$ & 10.82 & 9.99 \\
\hline September-03 & $5,576.53$ & 821 & 2,291 & 0 & 127 & 12,676 & 135 & 7 & -71 & 352 & 0.38 & 0.02 & $-1,653.98$ & 10.53 & 7.43 \\
\hline October-03 & $5,573.11$ & 730 & 1,783 & 0 & 0 & 11,566 & 83 & 5 & -21 & 327 & 0.25 & 0.02 & $-1,110.28$ & 10.24 & 2.16 \\
\hline November-03 & $5,574.98$ & 734 & 119 & 0 & 0 & 12,166 & 11 & 24 & 28 & 326 & 0.03 & 0.07 & 600.35 & 10.24 & 2.84 \\
\hline December-03 & $5,577.22$ & 809 & 123 & 0 & 0 & 12,907 & 2 & 37 & -19 & 336 & 0.01 & 0.11 & 740.62 & 10.23 & 1.95 \\
\hline January-04 & $5,579.04$ & 730 & 123 & 0 & 0 & 13,527 & 0 & 4 & -9 & 346 & 0.00 & 0.01 & 619.71 & 10.03 & 0.87 \\
\hline February-04 & $5,580.66$ & 639 & 115 & 0 & 0 & 14,092 & 7 & 20 & -29 & 355 & 0.02 & 0.06 & 565.66 & 10.20 & 3.01 \\
\hline March-04 & $5,582.87$ & 928 & 123 & 0 & 0 & 14,886 & 79 & 1 & -67 & 365 & 0.22 & 0.00 & 793.79 & 10.41 & 6.96 \\
\hline April-04 & $5,588.91$ & 3,320 & 835 & 0 & 0 & 17,194 & 103 & 35 & 108 & 387 & 0.27 & 0.09 & $2,307.66$ & 10.21 & 11.06 \\
\hline May-04 & $5,594.58$ & 5,770 & 3,741 & 236 & 0 & 19,559 & 190 & 9 & -754 & 429 & 0.44 & 0.02 & $2,365.43$ & 10.16 & 76.62 \\
\hline June-04 & $5,590.69$ & 1,880 & 3,227 & 62 & 44 & 17,916 & 239 & 43 & -6 & 429 & 0.56 & 0.10 & $-1,643.03$ & 10.46 & 0.62 \\
\hline July-04 & $5,587.08$ & 2,010 & 2,878 & 93 & 184 & 16,474 & 266 & 8 & 39 & 404 & 0.66 & 0.02 & $-1,442.47$ & 10.66 & 4.21 \\
\hline August-04 & $5,579.8$ & 1,490 & 3,416 & 0 & 417 & 13,790 & 202 & 6 & 144 & 375 & 0.54 & 0.02 & $-2,683.35$ & 10.76 & 15.53 \\
\hline September-04 & $5,574.96$ & 1,040 & 2,124 & 0 & 235 & 12,160 & 119 & 49 & 241 & 341 & 0.35 & 0.14 & $-1,630.53$ & 10.78 & 25.96 \\
\hline October-04 & $5,575.2$ & 1,670 & 1,543 & 0 & 0 & 12,238 & 54 & 113 & 108 & 324 & 0.17 & 0.35 & 78.19 & 10.48 & 11.28 \\
\hline November-04 & $5,581.67$ & 2,180 & 119 & 0 & 0 & 14,452 & 14 & 42 & -125 & 350 & 0.04 & 0.12 & $2,213.86$ & 10.12 & 12.61 \\
\hline December-04 & $5,584.97$ & 1,400 & 123 & 0 & 0 & 15,665 & 5 & 8 & 67 & 375 & 0.01 & 0.02 & $1,213.04$ & 10.05 & 6.69 \\
\hline January-05 & $5,587.89$ & 1,160 & 123 & 0 & 0 & 16,789 & 6 & 66 & -27 & 392 & 0.01 & 0.17 & $1,124.35$ & 10.29 & 2.80 \\
\hline February-05 & $5,587.29$ & 1,010 & 1,269 & 0 & 0 & 16,554 & 16 & 19 & -21 & 398 & 0.04 & 0.05 & -235.06 & 10.13 & 2.17 \\
\hline March-05 & $5,586.86$ & 1,310 & 1,537 & 0 & 0 & 16,387 & 51 & 45 & -66 & 394 & 0.13 & 0.11 & -167.17 & 10.31 & 6.77 \\
\hline April-05 & $5,589.9$ & 5,330 & 4,106 & 74 & 0 & 17,592 & 99 & 39 & -115 & 395 & 0.25 & 0.10 & $1,205.32$ & 10.13 & 11.67 \\
\hline May-05 & $5,604.07$ & 17,120 & 10,284 & 253 & 0 & 23,999 & 191 & 58 & 43 & 440 & 0.43 & 0.13 & $6,406.86$ & 10.07 & 4.28 \\
\hline June-05 & $5,608.06$ & 10,760 & 9,749 & 222 & 0 & 25,941 & 259 & 37 & $-1,374$ & 521 & 0.50 & 0.07 & $1,941.49$ & 10.13 & 139.20 \\
\hline July-05 & $5,605.42$ & 2,890 & 4,005 & 29 & 0 & 24,601 & 364 & 3 & -164 & 515 & 0.71 & 0.01 & $-1,339.84$ & 10.42 & 17.13 \\
\hline August-05 & $5,603.61$ & 2,660 & 3,140 & 21 & 58 & 23,708 & 275 & 28 & 87 & 499 & 0.55 & 0.06 & -893.04 & 10.51 & 9.15 \\
\hline September-05 & $5,600.62$ & 1,410 & 2,606 & 0 & 0 & 22,277 & 177 & 59 & 116 & 483 & 0.37 & 0.12 & $-1,430.37$ & 10.41 & 12.10 \\
\hline October-05 & $5,599.22$ & 1,330 & 1,837 & 0 & 0 & 21,627 & 89 & 51 & 105 & 470 & 0.19 & 0.11 & -650.76 & 10.35 & 10.92 \\
\hline November-05 & $5,598.88$ & 1,190 & 1,212 & 0 & 0 & 21,470 & 26 & 14 & 122 & 465 & 0.06 & 0.03 & -156.23 & 10.16 & 12.43 \\
\hline
\end{tabular}


Table 1. Reservoir data, evaporation, and calculated recharge and discharge from Red Fleet Reservoir, Utah, 1980-2015. - Continued

[AVWTP, Ashley Valley Water Treatment Plant; $\sigma$, sigma (standard deviation); - , no data]

\begin{tabular}{|c|c|c|c|c|c|c|c|c|c|c|c|c|c|c|c|}
\hline \multirow{2}{*}{ Month/year } & \multirow{2}{*}{$\begin{array}{l}\text { Reservoir } \\
\text { altitude } \\
\text { (feet) }\end{array}$} & \multirow{2}{*}{$\begin{array}{c}\text { Monthly } \\
\text { Big Brush } \\
\text { Creek } \\
\text { inflow } \\
\text { (acre-feet) }\end{array}$} & \multirow{2}{*}{$\begin{array}{l}\text { Monthly } \\
\text { Red Fleet } \\
\text { Dam out- } \\
\text { flow } \\
\text { (acre-feet) }\end{array}$} & \multicolumn{2}{|c|}{$\begin{array}{c}\text { Monthly pumped } \\
\text { outflows } \\
\text { (acre-feet) }\end{array}$} & \multirow{2}{*}{$\begin{array}{c}\text { Res- } \\
\text { ervoir } \\
\text { storage } \\
\text { (acre- } \\
\text { feet) }\end{array}$} & \multirow{2}{*}{$\begin{array}{c}\text { Monthly } \\
\text { evapora- } \\
\text { tion } \\
\text { (acre- } \\
\text { feet) }\end{array}$} & \multirow{2}{*}{$\begin{array}{c}\text { Monthly } \\
\text { precipita- } \\
\text { tion } \\
\text { (acre- } \\
\text { feet) }\end{array}$} & \multirow{2}{*}{$\begin{array}{c}\text { Monthly } \\
\text { groundwater } \\
\text { recharge/ } \\
\text { discharge } \\
\text { (-) } \\
\text { (acre-feet) }\end{array}$} & \multirow{2}{*}{$\begin{array}{l}\text { Res- } \\
\text { ervoir } \\
\text { surface } \\
\text { area } \\
\text { (acres) }\end{array}$} & \multirow{2}{*}{$\begin{array}{c}\text { Monthly } \\
\text { evapora- } \\
\text { tion rate } \\
\text { (feet) }\end{array}$} & \multirow{2}{*}{$\begin{array}{c}\text { Monthly } \\
\text { precipita- } \\
\text { tion rate } \\
\text { (feet) }\end{array}$} & \multirow{2}{*}{$\begin{array}{c}\text { Monthly } \\
\text { reservoir } \\
\text { storage } \\
\text { change } \\
\text { (acre-feet) }\end{array}$} & \multirow{2}{*}{$\begin{array}{c}\text { Monthly } \\
\text { groundwater } \\
\text { recharge/ } \\
\text { discharge un- } \\
\text { certainty, } 2 \sigma \\
\text { (percent) }\end{array}$} & \multirow{2}{*}{$\begin{array}{c}\text { Monthly } \\
\text { groundwater } \\
\text { recharge/ } \\
\text { discharge un- } \\
\text { certainty, } 2 \sigma \\
\text { (acre-feet) }\end{array}$} \\
\hline & & & & AVWTP & $\begin{array}{l}\text { Irriga- } \\
\text { tion }\end{array}$ & & & & & & & & & & \\
\hline December-05 & $5,598.82$ & 936 & 952 & 0 & 0 & 21,443 & 6 & 6 & 11 & 464 & 0.01 & 0.01 & -27.50 & 10.06 & 1.15 \\
\hline January-06 & $5,599.77$ & 875 & 476 & 0 & 0 & 21,881 & 6 & 27 & -18 & 466 & 0.01 & 0.06 & 437.96 & 10.18 & 1.82 \\
\hline February-06 & $5,601.2$ & 833 & 139 & 0 & 0 & 22,551 & 15 & 0 & 9 & 474 & 0.03 & 0.00 & 669.67 & 10.09 & 0.91 \\
\hline March-06 & $5,603.05$ & 958 & 154 & 0 & 0 & 23,436 & 55 & 89 & -47 & 484 & 0.11 & 0.18 & 885.11 & 10.67 & 5.01 \\
\hline April-06 & $5,602.33$ & 4,290 & 4,363 & 68 & 0 & 23,089 & 140 & 27 & 94 & 490 & 0.29 & 0.06 & -347.00 & 10.18 & 9.53 \\
\hline May-06 & $5,606.41$ & 9,020 & 5,881 & 371 & 0 & 25,098 & 246 & 6 & 519 & 500 & 0.49 & 0.01 & $2,009.39$ & 10.14 & 52.62 \\
\hline June-06 & $5,602.86$ & 2,610 & 3,499 & 84 & 243 & 23,344 & 364 & 34 & 208 & 505 & 0.72 & 0.07 & $-1,754.27$ & 10.75 & 22.38 \\
\hline July-06 & $5,596.88$ & 2,130 & 3,796 & 14 & 696 & 20,566 & 353 & 5 & 54 & 470 & 0.75 & 0.01 & $-2,778.19$ & 11.08 & 5.96 \\
\hline August-06 & $5,589.42$ & 1,250 & 2,850 & 10 & 963 & 17,400 & 257 & 19 & 354 & 429 & 0.60 & 0.05 & $-3,165.42$ & 11.46 & 40.57 \\
\hline September-06 & $5,587.46$ & 1,050 & 1,317 & 0 & 180 & 16,621 & 145 & 64 & 252 & 399 & 0.36 & 0.16 & -779.64 & 11.10 & 27.94 \\
\hline October-06 & $5,586.87$ & 1,070 & 1,192 & 0 & 0 & 16,391 & 71 & 105 & 141 & 393 & 0.18 & 0.27 & -229.68 & 10.66 & 15.05 \\
\hline November-06 & $5,589.01$ & 1,000 & 137 & 0 & 0 & 17,234 & 26 & 13 & 7 & 400 & 0.07 & 0.03 & 842.85 & 10.19 & 0.67 \\
\hline December-06 & $5,590.91$ & 952 & 141 & 0 & 0 & 18,005 & 2 & 12 & 48 & 411 & 0.01 & 0.03 & 771.40 & 10.08 & 4.87 \\
\hline January-07 & $5,592.57$ & 823 & 141 & 0 & 0 & 18,697 & 0 & 11 & 1 & 421 & 0.00 & 0.03 & 691.88 & 10.07 & 0.08 \\
\hline February-07 & $5,594.09$ & 688 & 128 & 0 & 0 & 19,343 & 17 & 13 & -90 & 431 & 0.04 & 0.03 & 646.44 & 10.20 & 9.16 \\
\hline March-07 & $5,596.46$ & 1,290 & 141 & 0 & 0 & 20,379 & 81 & 7 & 40 & 441 & 0.18 & 0.02 & $1,035.20$ & 10.35 & 4.11 \\
\hline April-07 & $5,600.28$ & 4,990 & 1,759 & 130 & 0 & 22,118 & 127 & 27 & 1,261 & 463 & 0.27 & 0.06 & $1,739.64$ & 10.18 & 128.34 \\
\hline May-07 & $5,607.89$ & 9,010 & 3,588 & 289 & 0 & 25,853 & 235 & 45 & 1,209 & 508 & 0.46 & 0.09 & $3,734.76$ & 10.17 & 122.89 \\
\hline June-07 & $5,604.45$ & 3,480 & 3,777 & 149 & 544 & 24,120 & 361 & 5 & 387 & 517 & 0.70 & 0.01 & $-1,733.37$ & 10.91 & 42.22 \\
\hline July-07 & $5,598.42$ & 2,200 & 3,390 & 13 & 1,078 & 21,260 & 377 & 8 & 210 & 480 & 0.79 & 0.02 & $-2,859.50$ & 11.47 & 24.06 \\
\hline August-07 & $5,591.78$ & 1,130 & 2,876 & 9 & 460 & 18,366 & 292 & 9 & 396 & 441 & 0.66 & 0.02 & $-2,893.80$ & 10.99 & 43.50 \\
\hline September-07 & $5,585.97$ & 1,010 & 2,037 & 17 & 748 & 16,045 & 170 & 15 & 374 & 402 & 0.42 & 0.04 & $-2,321.39$ & 11.48 & 42.94 \\
\hline October-07 & $5,585.32$ & 1,170 & 1,125 & 0 & 0 & 15,797 & 76 & 4 & 221 & 385 & 0.20 & 0.01 & -247.91 & 10.30 & 22.80 \\
\hline November-07 & $5,587.11$ & 958 & 149 & 0 & 0 & 16,484 & 30 & 0 & 92 & 390 & 0.08 & 0.00 & 686.99 & 10.16 & 9.38 \\
\hline December-07 & $5,588.89$ & 718 & 154 & 0 & 0 & 17,186 & 0 & 75 & -62 & 400 & 0.00 & 0.19 & 701.69 & 10.46 & 6.53 \\
\hline January-08 & $5,590.57$ & 708 & 154 & 0 & 0 & 17,865 & 0 & 16 & -110 & 410 & 0.00 & 0.04 & 679.72 & 10.10 & 11.10 \\
\hline February- 08 & $5,592.41$ & 672 & 144 & 0 & 0 & 18,630 & 2 & 29 & -209 & 420 & 0.01 & 0.07 & 764.29 & 10.20 & 21.31 \\
\hline March-08 & $5,594.3$ & 768 & 154 & 0 & 0 & 19,434 & 36 & 33 & -194 & 431 & 0.08 & 0.08 & 804.08 & 10.39 & 20.11 \\
\hline
\end{tabular}


[AVWTP, Ashley Valley Water Treatment Plant; $\sigma$, sigma (standard deviation); 一, no data]

\begin{tabular}{|c|c|c|c|c|c|c|c|c|c|c|c|c|c|c|c|}
\hline \multirow{2}{*}{ Month/year } & \multirow{2}{*}{$\begin{array}{l}\text { Reservoir } \\
\text { altitude } \\
\text { (feet) }\end{array}$} & \multirow{2}{*}{$\begin{array}{l}\text { Monthly } \\
\text { Big Brush } \\
\text { Creek } \\
\text { inflow } \\
\text { (acre-feet) }\end{array}$} & \multirow{2}{*}{$\begin{array}{l}\text { Monthly } \\
\text { Red Fleet } \\
\text { Dam out- } \\
\text { flow } \\
\text { (acre-feet) }\end{array}$} & \multicolumn{2}{|c|}{$\begin{array}{c}\text { Monthly pumped } \\
\text { outflows } \\
\text { (acre-feet) }\end{array}$} & \multirow{2}{*}{$\begin{array}{c}\text { Res- } \\
\text { ervoir } \\
\text { storage } \\
\text { (acre- } \\
\text { feet) }\end{array}$} & \multirow{2}{*}{$\begin{array}{c}\text { Monthly } \\
\text { evapora- } \\
\text { tion } \\
\text { (acre- } \\
\text { feet) }\end{array}$} & \multirow{2}{*}{$\begin{array}{l}\text { Monthly } \\
\text { precipita- } \\
\text { tion } \\
\text { (acre- } \\
\text { feet) }\end{array}$} & \multirow{2}{*}{$\begin{array}{c}\text { Monthly } \\
\text { groundwater } \\
\text { recharge/ } \\
\text { discharge } \\
\text { (-) } \\
\text { (acre-feet) }\end{array}$} & \multirow{2}{*}{$\begin{array}{l}\text { Res- } \\
\text { ervoir } \\
\text { surface } \\
\text { area } \\
\text { (acres) }\end{array}$} & \multirow{2}{*}{$\begin{array}{l}\text { Monthly } \\
\text { evapora- } \\
\text { tion rate } \\
\text { (feet) }\end{array}$} & \multirow{2}{*}{$\begin{array}{l}\text { Monthly } \\
\text { precipita- } \\
\text { tion rate } \\
\text { (feet) }\end{array}$} & \multirow{2}{*}{$\begin{array}{l}\text { Monthly } \\
\text { reservoir } \\
\text { storage } \\
\text { change } \\
\text { (acre-feet) }\end{array}$} & \multirow{2}{*}{$\begin{array}{l}\text { Monthly } \\
\text { groundwater } \\
\text { recharge/ } \\
\text { discharge un- } \\
\text { certainty, } 2 \sigma \\
\text { (percent) }\end{array}$} & \multirow{2}{*}{$\begin{array}{l}\text { Monthly } \\
\text { groundwater } \\
\text { recharge/ } \\
\text { discharge un- } \\
\text { certainty, } 2 \sigma \\
\text { (acre-feet) }\end{array}$} \\
\hline & & & & AVWTP & $\begin{array}{c}\text { Irriga- } \\
\text { tion }\end{array}$ & & & & & & & & & & \\
\hline April-08 & $5,595.76$ & 1,260 & 481 & 40 & 0 & 20,069 & 91 & 15 & 27 & 441 & 0.21 & 0.03 & 635.58 & 10.42 & 2.86 \\
\hline May-08 & $5,605.48$ & 10,250 & 4,731 & 304 & 0 & 24,631 & 195 & 0 & 459 & 474 & 0.41 & 0.00 & $4,561.38$ & 10.10 & 46.34 \\
\hline June-08 & $5,607.77$ & 7,340 & 5,891 & 222 & 0 & 25,791 & 334 & 62 & -206 & 520 & 0.64 & 0.12 & $1,160.52$ & 10.26 & 21.11 \\
\hline July-08 & $5,603.14$ & 2,310 & 3,771 & 22 & 379 & 23,479 & 380 & 6 & 76 & 505 & 0.75 & 0.01 & $-2,312.04$ & 10.83 & 8.26 \\
\hline August-08 & $5,596.25$ & 1,450 & 3,507 & 24 & 639 & 20,286 & 301 & 69 & 242 & 471 & 0.64 & 0.15 & $-3,193.70$ & 11.10 & 26.90 \\
\hline September-08 & $5,592.93$ & 851 & 1,781 & 24 & 70 & 18,849 & 159 & 38 & 291 & 438 & 0.36 & 0.09 & $-1,436.68$ & 10.61 & 30.90 \\
\hline October-08 & $5,590.88$ & 1,010 & 1,517 & 0 & 0 & 17,994 & 86 & 25 & 287 & 420 & 0.20 & 0.06 & -854.77 & 10.32 & 29.58 \\
\hline November-08 & $5,592.48$ & 875 & 149 & 0 & 0 & 18,659 & 29 & 21 & 53 & 421 & 0.07 & 0.05 & 665.04 & 10.29 & 5.47 \\
\hline December-08 & $5,594.16$ & 837 & 154 & 0 & 0 & 19,374 & 5 & 38 & 2 & 430 & 0.01 & 0.09 & 714.39 & 10.24 & 0.22 \\
\hline January-09 & $5,595.97$ & 970 & 154 & 0 & 0 & 20,162 & 2 & 40 & 66 & 441 & 0.00 & 0.09 & 788.33 & 10.21 & 6.70 \\
\hline February-09 & $5,597.55$ & 815 & 139 & 0 & 0 & 20,866 & 12 & 7 & -34 & 451 & 0.03 & 0.02 & 704.15 & 10.11 & 3.39 \\
\hline March-09 & $5,598.94$ & 833 & 154 & 0 & 0 & 21,498 & 62 & 9 & -5 & 460 & 0.13 & 0.02 & 631.93 & 10.42 & 0.56 \\
\hline April-09 & $5,598.9$ & 1,390 & 1,202 & 42 & 0 & 21,480 & 113 & 41 & 93 & 465 & 0.24 & 0.09 & -18.35 & 10.55 & 9.83 \\
\hline May-09 & $5,607.97$ & 9,260 & 4,413 & 275 & 0 & 25,894 & 238 & 22 & -58 & 497 & 0.48 & 0.05 & $4,414.65$ & 10.14 & 5.90 \\
\hline June-09 & $5,608.12$ & 7,060 & 4,344 & 139 & 0 & 25,972 & 312 & 60 & 2,248 & 524 & 0.60 & 0.11 & 77.38 & 10.31 & 231.73 \\
\hline July-09 & $5,606.15$ & 4,380 & 4,552 & 68 & 101 & 24,967 & 376 & 6 & 294 & 519 & 0.72 & 0.01 & $-1,004.77$ & 10.46 & 30.77 \\
\hline August-09 & $5,601.43$ & 2,350 & 3,529 & 0 & 643 & 22,659 & 298 & 21 & 210 & 496 & 0.60 & 0.04 & $-2,307.42$ & 11.05 & 23.19 \\
\hline September-09 & $5,598.36$ & 1,040 & 1,930 & 38 & 116 & 21,233 & 206 & 43 & 220 & 468 & 0.44 & 0.09 & $-1,426.60$ & 10.76 & 23.66 \\
\hline October-09 & 5,597 & 758 & 1,142 & 0 & 0 & 20,619 & 74 & 24 & 179 & 455 & 0.16 & 0.05 & -613.64 & 10.37 & 18.57 \\
\hline November-09 & $5,597.7$ & 738 & 417 & 0 & 0 & 20,934 & 28 & 14 & -7 & 455 & 0.06 & 0.03 & 314.45 & 10.28 & 0.75 \\
\hline December-09 & $5,598.3$ & 728 & 430 & 0 & 0 & 21,206 & 1 & 42 & 67 & 459 & 0.00 & 0.09 & 271.88 & 10.29 & 6.94 \\
\hline January-10 & $5,598.95$ & 728 & 430 & 0 & 0 & 21,503 & 1 & 22 & 22 & 463 & 0.00 & 0.05 & 297.00 & 10.15 & 2.19 \\
\hline February-10 & $5,599.28$ & 609 & 389 & 0 & 0 & 21,654 & 0 & 6 & 74 & 465 & 0.00 & 0.01 & 151.77 & 10.05 & 7.47 \\
\hline March-10 & $5,599.68$ & 571 & 430 & 0 & 0 & 21,839 & 47 & 20 & -71 & 468 & 0.10 & 0.04 & 184.85 & 10.54 & 7.46 \\
\hline April-10 & $5,601.66$ & 1,870 & 716 & 44 & 0 & 22,769 & 116 & 55 & 119 & 472 & 0.25 & 0.12 & 929.49 & 10.46 & 12.46 \\
\hline May-10 & $5,607.64$ & 7,960 & 4,120 & 265 & 0 & 25,725 & 174 & 25 & 471 & 499 & 0.35 & 0.05 & $2,955.87$ & 10.13 & 47.66 \\
\hline June-10 & $5,607.12$ & 7,270 & 6,799 & 295 & 0 & 25,459 & 320 & 67 & 188 & 522 & 0.61 & 0.13 & -266.03 & 10.26 & 19.33 \\
\hline July-10 & $5,603.22$ & 2,590 & 3,695 & 57 & 227 & 23,518 & 351 & 36 & 236 & 503 & 0.70 & 0.07 & $-1,940.43$ & 10.69 & 25.27 \\
\hline
\end{tabular}


Table 1. Reservoir data, evaporation, and calculated recharge and discharge from Red Fleet Reservoir, Utah, 1980-2015. - Continued

[AVWTP, Ashley Valley Water Treatment Plant; $\sigma$, sigma (standard deviation); - , no data]

\begin{tabular}{|c|c|c|c|c|c|c|c|c|c|c|c|c|c|c|c|}
\hline \multirow{2}{*}{ Month/year } & \multirow{2}{*}{$\begin{array}{l}\text { Reservoir } \\
\text { altitude } \\
\text { (feet) }\end{array}$} & \multirow{2}{*}{$\begin{array}{c}\text { Monthly } \\
\text { Big Brush } \\
\text { Creek } \\
\text { inflow } \\
\text { (acre-feet) }\end{array}$} & \multirow{2}{*}{$\begin{array}{c}\text { Monthly } \\
\text { Red Fleet } \\
\text { Dam out- } \\
\text { flow } \\
\text { (acre-feet) }\end{array}$} & \multicolumn{2}{|c|}{$\begin{array}{c}\text { Monthly pumped } \\
\text { outflows } \\
\text { (acre-feet) }\end{array}$} & \multirow{2}{*}{$\begin{array}{c}\text { Res- } \\
\text { ervoir } \\
\text { storage } \\
\text { (acre- } \\
\text { feet) }\end{array}$} & \multirow{2}{*}{$\begin{array}{c}\text { Monthly } \\
\text { evapora- } \\
\text { tion } \\
\text { (acre- } \\
\text { feet) }\end{array}$} & \multirow{2}{*}{$\begin{array}{c}\text { Monthly } \\
\text { precipita- } \\
\text { tion } \\
\text { (acre- } \\
\text { feet) }\end{array}$} & \multirow{2}{*}{$\begin{array}{c}\text { Monthly } \\
\text { groundwater } \\
\text { recharge/ } \\
\text { discharge } \\
\text { (-) } \\
\text { (acre-feet) }\end{array}$} & \multirow{2}{*}{$\begin{array}{l}\text { Res- } \\
\text { ervoir } \\
\text { surface } \\
\text { area } \\
\text { (acres) }\end{array}$} & \multirow{2}{*}{$\begin{array}{c}\text { Monthly } \\
\text { evapora- } \\
\text { tion rate } \\
\text { (feet) }\end{array}$} & \multirow{2}{*}{$\begin{array}{c}\text { Monthly } \\
\text { precipita- } \\
\text { tion rate } \\
\text { (feet) }\end{array}$} & \multirow{2}{*}{$\begin{array}{c}\text { Monthly } \\
\text { reservoir } \\
\text { storage } \\
\text { change } \\
\text { (acre-feet) }\end{array}$} & \multirow{2}{*}{$\begin{array}{c}\text { Monthly } \\
\text { groundwater } \\
\text { recharge/ } \\
\text { discharge un- } \\
\text { certainty, } 2 \sigma \\
\text { (percent) }\end{array}$} & \multirow{2}{*}{$\begin{array}{c}\text { Monthly } \\
\text { groundwater } \\
\text { recharge/ } \\
\text { discharge un } \\
\text { certainty, } 2 \sigma \\
\text { (acre-feet) }\end{array}$} \\
\hline & & & & AVWTP & $\begin{array}{l}\text { Irriga- } \\
\text { tion }\end{array}$ & & & & & & & & & & \\
\hline August-10 & $5,596.46$ & 1,490 & 3,441 & 36 & 617 & 20,379 & 271 & 30 & 294 & 471 & 0.58 & 0.06 & $-3,139.42$ & 11.02 & 32.36 \\
\hline September-10 & $5,590.86$ & 916 & 2,154 & 41 & 625 & 17,986 & 188 & 12 & 312 & 431 & 0.44 & 0.03 & $-2,392.75$ & 11.30 & 35.26 \\
\hline October-10 & $5,590.77$ & 889 & 745 & 0 & 0 & 17,949 & 84 & 56 & 154 & 413 & 0.20 & 0.14 & -36.96 & 10.77 & 16.55 \\
\hline November-10 & $5,592.35$ & 762 & 149 & 0 & 0 & 18,605 & 23 & 29 & -36 & 420 & 0.05 & 0.07 & 655.63 & 10.32 & 3.76 \\
\hline December-10 & $5,594.21$ & 783 & 154 & 0 & 0 & 19,395 & 5 & 97 & -69 & 430 & 0.01 & 0.23 & 790.50 & 10.56 & 7.31 \\
\hline January-11 & $5,595.74$ & 681 & 154 & 0 & 0 & 20,061 & 0 & 0 & -138 & 441 & 0.00 & 0.00 & 665.56 & 10.00 & 13.83 \\
\hline February-11 & $5,596.91$ & 559 & 139 & 0 & 0 & 20,579 & 1 & 22 & -78 & 448 & 0.00 & 0.05 & 518.37 & 10.19 & 7.93 \\
\hline March-11 & $5,596.08$ & 736 & 1,220 & 0 & 0 & 20,210 & 41 & 16 & -140 & 451 & 0.09 & 0.04 & -368.58 & 10.24 & 14.38 \\
\hline April-11 & $5,594.8$ & 1,450 & 1,864 & 85 & 0 & 19,650 & 83 & 42 & 19 & 443 & 0.19 & 0.09 & -560.34 & 10.31 & 1.99 \\
\hline May-11 & $5,601.72$ & 8,470 & 6,672 & 291 & 0 & 22,797 & 139 & 95 & $-1,685$ & 456 & 0.31 & 0.21 & $3,147.13$ & 10.12 & 170.56 \\
\hline June-11 & $5,607.96$ & 11,640 & 9,808 & 381 & 0 & 25,889 & 273 & 20 & $-1,895$ & 510 & 0.54 & 0.04 & $3,091.90$ & 10.12 & 191.66 \\
\hline July-11 & $5,607.19$ & 4,020 & 4,721 & 174 & 0 & 25,494 & 327 & 110 & -697 & 522 & 0.63 & 0.21 & -394.89 & 10.45 & 72.83 \\
\hline August-11 & $5,602.82$ & 2,590 & 3,874 & 0 & 394 & 23,324 & 325 & 23 & 190 & 505 & 0.64 & 0.05 & $-2,169.74$ & 10.79 & 20.51 \\
\hline September-11 & $5,599.21$ & 1,460 & 2,664 & 0 & 0 & 21,622 & 202 & 4 & 300 & 476 & 0.43 & 0.01 & $-1,702.41$ & 10.34 & 31.04 \\
\hline October-11 & $5,598.2$ & 1,270 & 1,476 & 0 & 0 & 21,160 & 83 & 68 & 242 & 461 & 0.18 & 0.15 & -461.99 & 10.45 & 25.28 \\
\hline November-11 & $5,598.21$ & 1,060 & 918 & 0 & 0 & 21,165 & 17 & 24 & 144 & 460 & 0.04 & 0.05 & 4.54 & 10.20 & 14.67 \\
\hline December-11 & $5,598.16$ & 986 & 922 & 0 & 0 & 21,142 & 1 & 8 & 93 & 460 & 0.00 & 0.02 & -22.71 & 10.05 & 9.31 \\
\hline January-12 & $5,599.44$ & 823 & 253 & 0 & 0 & 21,728 & 3 & 14 & -5 & 463 & 0.01 & 0.03 & 586.23 & 10.10 & 0.48 \\
\hline February-12 & $5,600.78$ & 720 & 144 & 0 & 0 & 22,353 & 12 & 26 & -34 & 472 & 0.03 & 0.06 & 624.44 & 10.25 & 3.50 \\
\hline March-12 & $5,602.44$ & 926 & 154 & 0 & 0 & 23,141 & 88 & 0 & -105 & 481 & 0.18 & 0.00 & 788.90 & 10.45 & 10.93 \\
\hline April-12 & $5,605.69$ & 3,920 & 2,001 & 237 & 38 & 24,736 & 149 & 19 & -80 & 497 & 0.30 & 0.04 & $1,594.35$ & 10.26 & 8.22 \\
\hline May-12 & $5,603.24$ & 2,630 & 3,324 & 464 & 13 & 23,528 & 240 & 3 & -201 & 503 & 0.48 & 0.01 & $-1,208.04$ & 10.32 & 20.70 \\
\hline June-12 & $5,598.3$ & 1,990 & 3,199 & 210 & 765 & 21,206 & 333 & 0 & -194 & 478 & 0.70 & 0.00 & $-2,322.24$ & 11.24 & 21.84 \\
\hline July-12 & $5,589.52$ & 825 & 3,503 & 39 & 644 & 17,440 & 293 & 26 & 137 & 434 & 0.68 & 0.06 & $-3,765.06$ & 11.06 & 15.13 \\
\hline August-12 & $5,579.05$ & 710 & 3,211 & 0 & 882 & 13,530 & 225 & 17 & 319 & 377 & 0.60 & 0.04 & $-3,910.41$ & 11.25 & 35.91 \\
\hline September-12 & $5,573.44$ & 676 & 2,099 & 0 & 0 & 11,671 & 136 & 59 & 360 & 336 & 0.40 & 0.18 & $-1,859.35$ & 10.40 & 37.49 \\
\hline October-12 & $5,574.88$ & 651 & 159 & 0 & 0 & 12,134 & 65 & 27 & -9 & 325 & 0.20 & 0.08 & 463.05 & 10.68 & 0.97 \\
\hline November-12 & $5,576.79$ & 706 & 148 & 0 & 0 & 12,763 & 25 & 5 & -91 & 334 & 0.07 & 0.01 & 629.10 & 10.20 & 9.33 \\
\hline
\end{tabular}


[AVWTP, Ashley Valley Water Treatment Plant; $\sigma$, sigma (standard deviation); - , no data]

\begin{tabular}{|c|c|c|c|c|c|c|c|c|c|c|c|c|c|c|c|}
\hline \multirow{2}{*}{ Month/year } & \multirow{2}{*}{$\begin{array}{l}\text { Reservoir } \\
\text { altitude } \\
\text { (feet) }\end{array}$} & \multirow{2}{*}{$\begin{array}{c}\text { Monthly } \\
\text { Big Brush } \\
\text { Creek } \\
\text { inflow } \\
\text { (acre-feet) }\end{array}$} & \multirow{2}{*}{$\begin{array}{l}\text { Monthly } \\
\text { Red Fleet } \\
\text { Dam out- } \\
\text { flow } \\
\text { (acre-feet) }\end{array}$} & \multicolumn{2}{|c|}{$\begin{array}{c}\text { Monthly pumped } \\
\text { outflows } \\
\text { (acre-feet) }\end{array}$} & \multirow{2}{*}{$\begin{array}{c}\text { Res- } \\
\text { ervoir } \\
\text { storage } \\
\text { (acre- } \\
\text { feet) }\end{array}$} & \multirow{2}{*}{$\begin{array}{c}\text { Monthly } \\
\text { evapora- } \\
\text { tion } \\
\text { (acre- } \\
\text { feet) }\end{array}$} & \multirow{2}{*}{$\begin{array}{l}\text { Monthly } \\
\text { precipita- } \\
\text { tion } \\
\text { (acre- } \\
\text { feet) }\end{array}$} & \multirow{2}{*}{$\begin{array}{c}\text { Monthly } \\
\text { groundwater } \\
\text { recharge/ } \\
\text { discharge } \\
\text { (-) } \\
\text { (acre-feet) }\end{array}$} & \multirow{2}{*}{$\begin{array}{l}\text { Res- } \\
\text { ervoir } \\
\text { surface } \\
\text { area } \\
\text { (acres) }\end{array}$} & \multirow{2}{*}{$\begin{array}{l}\text { Monthly } \\
\text { evapora- } \\
\text { tion rate } \\
\text { (feet) }\end{array}$} & \multirow{2}{*}{$\begin{array}{l}\text { Monthly } \\
\text { precipita- } \\
\text { tion rate } \\
\text { (feet) }\end{array}$} & \multirow{2}{*}{$\begin{array}{l}\text { Monthly } \\
\text { reservoir } \\
\text { storage } \\
\text { change } \\
\text { (acre-feet) }\end{array}$} & \multirow{2}{*}{$\begin{array}{l}\text { Monthly } \\
\text { groundwater } \\
\text { recharge/ } \\
\text { discharge un- } \\
\text { certainty, 2 } \sigma \\
\text { (percent) }\end{array}$} & \multirow{2}{*}{$\begin{array}{l}\text { Monthly } \\
\text { groundwater } \\
\text { recharge/ } \\
\text { discharge un- } \\
\text { certainty, } 2 \sigma \\
\text { (acre-feet) }\end{array}$} \\
\hline & & & & AVWTP & $\begin{array}{l}\text { Irriga- } \\
\text { tion }\end{array}$ & & & & & & & & & & \\
\hline December-12 & $5,578.62$ & 670 & 154 & 0 & 0 & 13,382 & 5 & 22 & -86 & 344 & 0.01 & 0.06 & 619.27 & 10.18 & 8.74 \\
\hline January-13 & $5,580.42$ & 661 & 154 & 0 & 0 & 14,008 & 0 & 29 & -90 & 353 & 0.00 & 0.08 & 625.48 & 10.20 & 9.18 \\
\hline February-13 & $5,581.56$ & 458 & 139 & 0 & 0 & 14,412 & 0 & 8 & -78 & 361 & 0.00 & 0.02 & 404.79 & 10.07 & 7.87 \\
\hline March-13 & $5,582.91$ & 586 & 154 & 0 & 0 & 14,901 & 47 & 20 & -83 & 368 & 0.13 & 0.05 & 488.27 & 10.51 & 8.70 \\
\hline April-13 & $5,583.59$ & 780 & 512 & 108 & 0 & 15,150 & 83 & 38 & -134 & 375 & 0.22 & 0.10 & 249.67 & 10.69 & 14.30 \\
\hline May-13 & $5,589.93$ & 5,480 & 3,205 & 357 & 0 & 17,605 & 210 & 25 & -721 & 391 & 0.54 & 0.07 & $2,454.14$ & 10.20 & 73.52 \\
\hline June-13 & $5,584.78$ & 1,870 & 3,249 & 359 & 0 & 15,593 & 327 & 0 & -54 & 399 & 0.82 & 0.00 & $-2,011.08$ & 10.42 & 5.60 \\
\hline July-13 & $5,576.92$ & 1,870 & 3,406 & 45 & 773 & 12,806 & 305 & 25 & 153 & 359 & 0.85 & 0.07 & $-2,787.08$ & 11.20 & 17.12 \\
\hline August-13 & $5,565.09$ & 720 & 2,959 & 45 & 881 & 9,161 & 200 & 14 & 294 & 307 & 0.65 & 0.04 & $-3,645.70$ & 11.29 & 33.16 \\
\hline September-13 & $5,562.32$ & 629 & 883 & 204 & 138 & 8,428 & 110 & 64 & 91 & 268 & 0.41 & 0.24 & -733.13 & 11.13 & 10.15 \\
\hline October-13 & $5,563.64$ & 762 & 478 & 0 & 0 & 8,783 & 50 & 33 & -88 & 268 & 0.18 & 0.12 & 355.03 & 10.49 & 9.21 \\
\hline November-13 & $5,566.29$ & 805 & 119 & 0 & 0 & 9,520 & 14 & 9 & -56 & 280 & 0.05 & 0.03 & 737.44 & 10.13 & 5.71 \\
\hline December-13 & $5,568.83$ & 823 & 123 & 0 & 0 & 10,258 & 0 & 41 & 2 & 293 & 0.00 & 0.14 & 738.48 & 10.24 & 0.24 \\
\hline January-14 & $5,570.83$ & 686 & 123 & 0 & 0 & 10,862 & 0 & 13 & -28 & 304 & 0.00 & 0.04 & 603.83 & 10.09 & 2.87 \\
\hline February-14 & $5,572.63$ & 617 & 111 & 0 & 0 & 11,414 & 17 & 4 & -59 & 313 & 0.06 & 0.01 & 552.11 & 10.17 & 6.00 \\
\hline March-14 & $5,574.57$ & 702 & 149 & 0 & 0 & 12,033 & 53 & 25 & -94 & 323 & 0.16 & 0.08 & 618.91 & 10.50 & 9.86 \\
\hline April-14 & $5,576.36$ & 1,710 & 1,034 & 6 & 0 & 12,620 & 97 & 19 & 5 & 332 & 0.29 & 0.06 & 586.44 & 10.34 & 0.52 \\
\hline May-14 & $5,579.86$ & 4,530 & 3,001 & 283 & 0 & 13,811 & 169 & 3 & -112 & 346 & 0.49 & 0.01 & $1,191.53$ & 10.19 & 11.37 \\
\hline June-14 & $5,573.43$ & 2,430 & 3,364 & 329 & 235 & 11,668 & 240 & 1 & 406 & 341 & 0.71 & 0.00 & $-2,143.72$ & 10.54 & 42.83 \\
\hline July-14 & $5,561.31$ & 1,520 & 3,481 & 218 & 441 & 8,112 & 240 & 15 & 710 & 290 & 0.83 & 0.05 & $-3,555.18$ & 10.74 & 76.24 \\
\hline August-14 & $5,555.97$ & 1,300 & 2,146 & 68 & 0 & 6,830 & 146 & 74 & 297 & 239 & 0.61 & 0.31 & $-1,282.73$ & 10.44 & 31.01 \\
\hline September-14 & $5,559.74$ & 1,610 & 837 & 0 & 0 & 7,757 & 108 & 59 & -204 & 246 & 0.44 & 0.24 & 926.99 & 10.47 & 21.33 \\
\hline October-14 & $5,563.88$ & 1,850 & 885 & 0 & 0 & 8,848 & 64 & 1 & -189 & 269 & 0.24 & 0.01 & $1,091.32$ & 10.17 & 19.21 \\
\hline November-14 & $5,567.45$ & 1,050 & 103 & 0 & 0 & 9,853 & 15 & 10 & -64 & 285 & 0.05 & 0.04 & $1,005.38$ & 10.12 & 6.46 \\
\hline December-14 & $5,570.37$ & 936 & 154 & 0 & 0 & 10,729 & 5 & 13 & -86 & 300 & 0.02 & 0.04 & 875.66 & 10.09 & 8.64 \\
\hline January-15 & $5,572.77$ & 807 & 154 & 0 & 0 & 11,458 & 4 & 8 & -72 & 313 & 0.01 & 0.03 & 729.49 & 10.07 & 7.30 \\
\hline February-15 & $5,574.62$ & 680 & 139 & 0 & 0 & 12,049 & 23 & 0 & -73 & 324 & 0.07 & 0.00 & 590.99 & 10.16 & 7.39 \\
\hline March-15 & $5,576.82$ & 839 & 154 & 0 & 0 & 12,773 & 66 & 44 & -60 & 334 & 0.20 & 0.13 & 723.43 & 10.60 & 6.40 \\
\hline
\end{tabular}


Table 1. Reservoir data, evaporation, and calculated recharge and discharge from Red Fleet Reservoir, Utah, 1980-2015. - Continued

[AVWTP, Ashley Valley Water Treatment Plant; $\sigma$, sigma (standard deviation); - , no data]

\begin{tabular}{|c|c|c|c|c|c|c|c|c|c|c|c|c|c|c|c|}
\hline \multirow{2}{*}{ Month/year } & \multirow{2}{*}{$\begin{array}{l}\text { Reservoir } \\
\text { altitude } \\
\text { (feet) }\end{array}$} & \multirow{2}{*}{$\begin{array}{c}\text { Monthly } \\
\text { Big Brush } \\
\text { Creek } \\
\text { inflow } \\
\text { (acre-feet) }\end{array}$} & \multirow{2}{*}{$\begin{array}{c}\text { Monthly } \\
\text { Red Fleet } \\
\text { Dam out- } \\
\text { flow } \\
\text { (acre-feet) }\end{array}$} & \multicolumn{2}{|c|}{$\begin{array}{c}\text { Monthly pumped } \\
\text { outflows } \\
\text { (acre-feet) }\end{array}$} & \multirow{2}{*}{$\begin{array}{c}\text { Res- } \\
\text { ervoir } \\
\text { storage } \\
\text { (acre- } \\
\text { feet) }\end{array}$} & \multirow{2}{*}{$\begin{array}{c}\text { Monthly } \\
\text { evapora- } \\
\text { tion } \\
\text { (acre- } \\
\text { feet) }\end{array}$} & \multirow{2}{*}{$\begin{array}{c}\text { Monthly } \\
\text { precipita- } \\
\text { tion } \\
\text { (acre- } \\
\text { feet) }\end{array}$} & \multirow{2}{*}{$\begin{array}{c}\text { Monthly } \\
\text { groundwater } \\
\text { recharge/ } \\
\text { discharge } \\
(-) \\
\text { (acre-feet) }\end{array}$} & \multirow{2}{*}{$\begin{array}{l}\text { Res- } \\
\text { ervoir } \\
\text { surface } \\
\text { area } \\
\text { (acres) }\end{array}$} & \multirow{2}{*}{$\begin{array}{l}\text { Monthly } \\
\text { evapora- } \\
\text { tion rate } \\
\text { (feet) }\end{array}$} & \multirow{2}{*}{$\begin{array}{c}\text { Monthly } \\
\text { precipita- } \\
\text { tion rate } \\
\text { (feet) }\end{array}$} & \multirow{2}{*}{$\begin{array}{l}\text { Monthly } \\
\text { reservoir } \\
\text { storage } \\
\text { change } \\
\text { (acre-feet) }\end{array}$} & \multirow{2}{*}{$\begin{array}{c}\text { Monthly } \\
\text { groundwater } \\
\text { recharge/ } \\
\text { discharge un- } \\
\text { certainty, } 2 \sigma \\
\text { (percent) }\end{array}$} & \multirow{2}{*}{$\begin{array}{c}\text { Monthly } \\
\text { groundwater } \\
\text { recharge/ } \\
\text { discharge un- } \\
\text { certainty, } 2 \sigma \\
\text { (acre-feet) }\end{array}$} \\
\hline & & & & AVWTP & $\begin{array}{c}\text { Irriga- } \\
\text { tion }\end{array}$ & & & & & & & & & & \\
\hline April-15 & $5,576.27$ & 1,510 & 1,688 & 0 & 0 & 12,590 & 98 & 9 & -84 & 337 & 0.29 & 0.03 & -183.01 & 10.31 & 8.66 \\
\hline May-15 & $5,591.77$ & 7,630 & 1,521 & 203 & 0 & 18,362 & 142 & 105 & 97 & 367 & 0.39 & 0.29 & $5,771.73$ & 10.16 & 9.87 \\
\hline June-15 & $5,598.77$ & 6,330 & 2,945 & 239 & 0 & 21,420 & 358 & 12 & -259 & 461 & 0.78 & 0.03 & $3,058.43$ & 10.29 & 26.61 \\
\hline July-15 & $5,594.48$ & 2,190 & 3,150 & 296 & 150 & 19,512 & 317 & 26 & 212 & 448 & 0.71 & 0.06 & $-1,908.53$ & 10.61 & 22.48 \\
\hline August-15 & $5,587.57$ & 1,530 & 3,511 & 166 & 81 & 16,665 & 275 & 15 & 359 & 417 & 0.66 & 0.04 & $-2,846.40$ & 10.44 & 37.44 \\
\hline September-15 & $5,583.12$ & 795 & 2,200 & 22 & 0 & 14,978 & 179 & 46 & 128 & 385 & 0.47 & 0.12 & $-1,687.59$ & 10.46 & 13.41 \\
\hline October-15 & $5,581.68$ & 903 & 1,088 & 0 & 0 & 14,455 & 94 & 22 & 266 & 366 & 0.26 & 0.06 & -522.09 & 10.44 & 27.74 \\
\hline November-15 & $5,583.47$ & 823 & 149 & 0 & 0 & 15,106 & 21 & 29 & 32 & 370 & 0.06 & 0.08 & 650.69 & 10.30 & 3.28 \\
\hline December-15 & $5,585.06$ & 654 & 154 & 0 & 0 & 15,699 & 0 & 19 & -74 & 379 & 0.00 & 0.05 & 592.70 & 10.13 & 7.47 \\
\hline Total & - & $1,050,000$ & 993,000 & 21,200 & 21,300 & - & 51,700 & 11,500 & $-39,900$ & - & - & - & - & - & - \\
\hline
\end{tabular}




\section{Meteorological Data}

Meteorological data utilized in this study have been collected at the Vernal Airport weather station (fig. 1) in Vernal, Utah, since the filling of Red Fleet Reservoir in 1980. The Vernal Airport weather station is approximately $10 \mathrm{mi}$ southwest of the reservoir at an elevation of 5,270 feet (ft) above sea level. Beginning in 2010, data from a Soil Climate Analysis Network (SCAN) Split Mountain weather station operated by the Natural Resources Conservation Service (NRCS) also were utilized. The NRCS SCAN Split Mountain weather station is approximately $13 \mathrm{mi}$ south of the reservoir (fig. 1) at an elevation of 4,844 ft above sea level. Data from both weather stations have been used for evaluating evaporation and precipitation, which are required for calculating monthly groundwater recharge/discharge from Red Fleet Reservoir. Parameters measured include air temperature, wind speed, precipitation, relative humidity, and incoming solar radiation. The solar-radiation and temperature data were used for calculating evaporation using the McGuinness and Bordne (1972) version of the Jensen-Haise method.

From May 1, 1980, to December 31, 2015, daily average air temperature ranged from -29 to 28 degrees Celsius $\left({ }^{\circ} \mathrm{C}\right)$ at the Vernal Airport. The coldest temperatures during the year typically were in December and January, when minimum air temperatures occasionally were below $-35^{\circ} \mathrm{C}$. The warmest temperatures were typically in July, when maximum air temperatures occasionally approached $40^{\circ} \mathrm{C}$. Daily average solar radiation ranged from 39 to 790 calories per square centimeter per day $\left(\mathrm{cm}^{2} / \mathrm{d}\right)$. The minimum daily averages are typically in December and January, and the maximum daily averages are typically in June and July. Temperature and solar-radiation data from the NRCS SCAN Split Mountain weather station from January 27, 2010, to December 31, 2015, were used for comparison to investigate spatial meteorological variability; during this period the average daily air temperature ranged from -25 to $28{ }^{\circ} \mathrm{C}$. Daily solar radiation ranged from 31 to 860 calories per $\mathrm{cm}^{2} / \mathrm{d}$. Cross-plotted average daily temperature data for the two sites between January 27, 2010, and December 31,2015, yielded a correlation coefficient of 0.97 . Cross-plotting the average daily solar-radiation data for the two sites for the same period yielded a correlation coefficient of 0.92 . On average, daily solar-radiation values recorded at Split Mountain were about 50 calories per $\mathrm{cm}^{2} / \mathrm{d}$ greater than at Vernal Airport. This is likely the result of numerous types of variability associated with solar-radiation measurements including individual sensor performance, latitudinal variation, and sensor orientation (level and free from shadow).

Monthly precipitation totals recorded at the Vernal Airport weather station from May 1980 through December 2015 were used to estimate precipitation at Red Fleet Reservoir; the monthly precipitation ranged from 0 to about $0.37 \mathrm{ft}$ (4.4 inches [in.]; table 1) and averaged about $0.07 \mathrm{ft}$ ( $0.8 \mathrm{in}$.). Average annual precipitation during the 36-year period from 1980 through 2015 was 9.3 in. (fig. 4). The years when annual precipitation was greater than

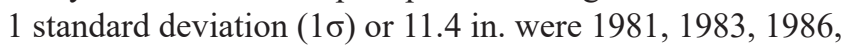
1997, 2010, and 2013, which is indicative of wetter than normal conditions near Red Fleet Reservoir. The years when annual precipitation was less than $1 \sigma$ or $7.1 \mathrm{in}$. were 1988 , 1989, 1991, 2001, 2002, 2007, and 2012, which is indicative of drier than normal conditions near Red Fleet Reservoir.

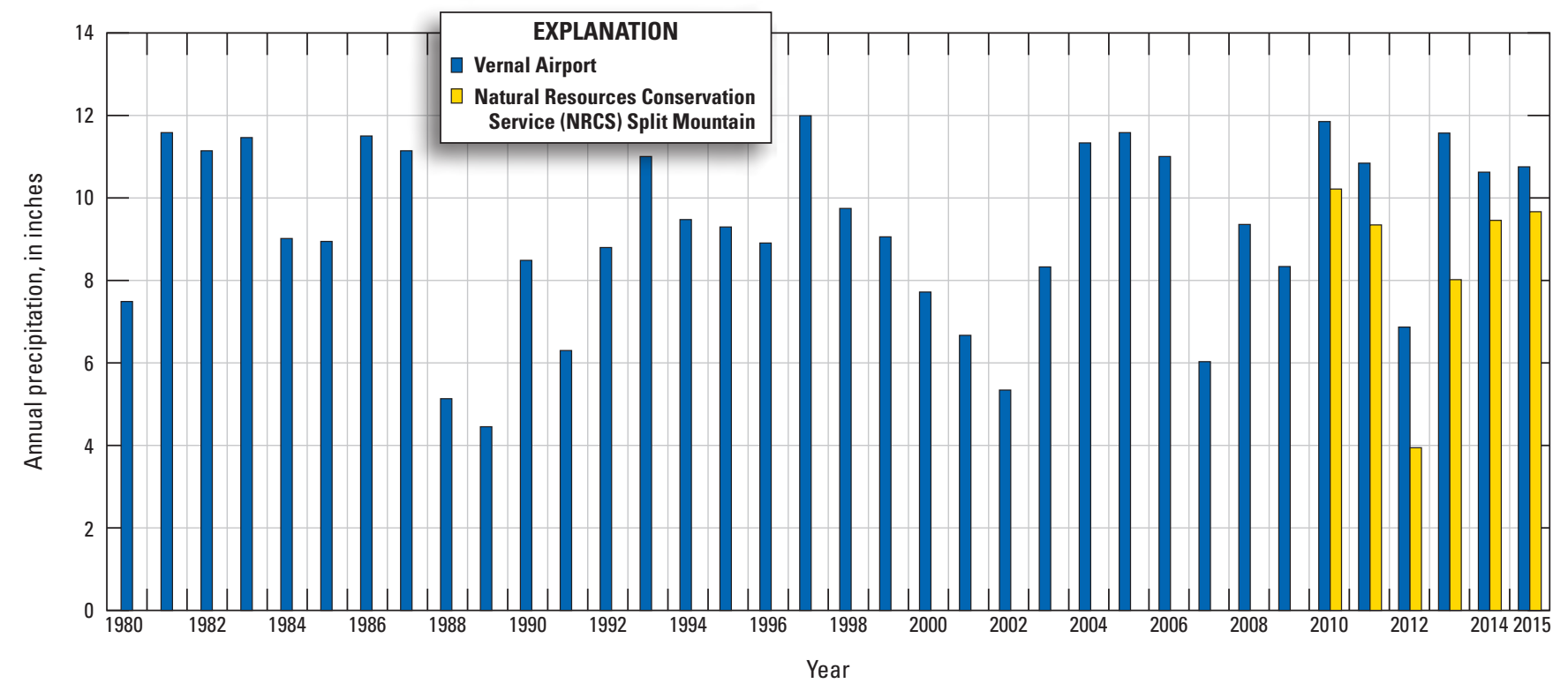

Figure 4. Annual precipitation at Vernal Airport and Natural Resources Conservation Service Split Mountain weather stations, Utah, 1980-2015. 


\section{Water-Level Data}

Water levels in four wells were recorded with Onset ${ }^{\circledR}$ Hobo water level data loggers from mid-August 2016 through September 2017 (fig. 5). The Red Fleet Reservoir stage recorded by UWCD is plotted on figure 5 with reference to water-level altitudes observed in nearby wells. A separate data logger was installed $10 \mathrm{ft}$ below land surface and about $40 \mathrm{ft}$ above the average water level inside monitoring well RF1 to record barometric pressure at the site for the same period (fig. 2). Barometric pressure data from this logger were used to make water-level corrections because of barometric fluctuations over the period of record. Regular electric-tape measurements were taken at each of the four wells to facilitate corrections to water levels recorded by the loggers.

The Red Fleet Reservoir stage observed between August 2016 and September 2017 fluctuated between about $5,585 \mathrm{ft}$ to about 5,605 $\mathrm{ft}$ with low stage during the late summer, and with the reservoir slowly filling through the winter and reaching high stage in the late spring/early summer following spring runoff. Water levels observed in well DH-109 follow the same trend as the altitude of the stage in Red Fleet Reservoir (fig. 5). Well DH-109 is on the west side of the reservoir near the eastern tip of a peninsula formed by the Frontier Sandstone that extends about 1,100 ft into the reservoir and was drilled by the BOR during the geologic investigations for the Red Fleet Dam site in the 1960s; DH-109 was drilled to a depth of $201 \mathrm{ft}$ below land surface and is open to the Frontier Sandstone and the Morrison Formation (Thompson, 1969). The water level observed in DH-109 was consistently about $3 \mathrm{ft}$ lower than the reservoir stage, indicating that the reservoir was likely recharging the Frontier Sandstone during the period of record.

Well RF2 is on the west side of the reservoir near the south end about 1,200 ft west of the nearest shore; RF2 was drilled and cased in September 2016 to a depth of $265 \mathrm{ft}$ below land surface. The bottom $20 \mathrm{ft}$ of RF2 is screened in the upper part of the Frontier Sandstone. Water levels in RF2 were about 15-30 ft lower than the stage observed in Red Fleet Reservoir, which indicates a downward groundwater-flow gradient in the Frontier Sandstone aquifer west of the reservoir during the period of record. Water levels in well RF2 remained relatively stable throughout the period of record with a slight increase from 5,578 ft in August 2016 to 5,580 ft in September 2017.

Well RF1 is on the west side of the reservoir near the inflow arm about $800 \mathrm{ft}$ west of the state park boat ramp and about $550 \mathrm{ft}$ from the nearest shore to the north. Water levels observed in well RF1 remained relatively stable throughout the period of record with a slight increase in water level from $5,639 \mathrm{ft}$ in August 2016 to 5,640 ft in September 2017. Well RF1 was drilled and cased in September 2016 to a depth of $100 \mathrm{ft}$ below land surface; the bottom $20 \mathrm{ft}$ of RF1 is screened in the Nugget Sandstone aquifer. The water levels in RF1 were about 35-50 ft higher than the stage observed in Red Fleet Reservoir, indicating that the Nugget Sandstone aquifer was discharging water to Red Fleet Reservoir during the observed period of record.

Water levels observed in well DH-111 were about $70-85 \mathrm{ft}$ higher than the stage observed in Red Fleet Reservoir over the same period. Well DH-111 is on the east side of the reservoir near Donkey Flats Road and was drilled by the BOR during the geologic investigations for the Red Fleet Dam site in the 1960s (fig. 2); DH-111 was drilled to a depth of $95 \mathrm{ft}$ below land surface and is open to the Nugget Sandstone aquifer (Thompson, 1969). The water levels observed in DH-111 were relatively stable with the exception of a waterlevel increase in February 2017. This increase in water level coincided with significant ponding from a mid-elevation snowmelt around the well at that time. Given the shallow depth to water (approximately $32 \mathrm{ft}$ below land surface), the increase in water level was likely the result of local recharge.

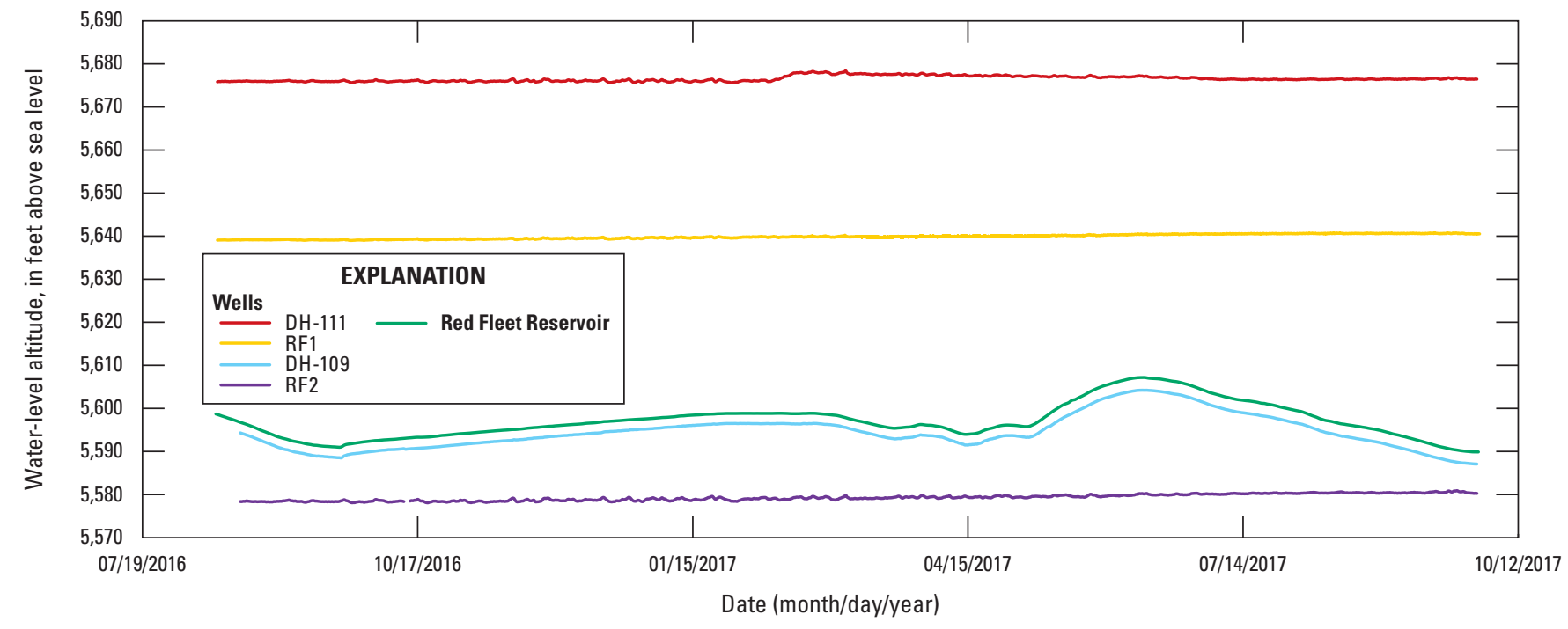

Figure 5. Water-level altitude in selected wells and Red Fleet Reservoir stage, Uintah County, Utah, 2016-17. 
Well DH-121, which was drilled by the BOR during the geologic investigations for the Red Fleet Dam site in the 1960s, is on the eastern peninsula that separates the Cottonwood Wash arm of Red Fleet Reservoir from the main body and wash (fig. 2). This well was drilled to a depth of $130 \mathrm{ft}$ below land surface and in September 2016, the water level was $49 \mathrm{ft}$ below land surface. The well is open to the Morrison Formation and Dakota Sandstone. Given the similar geographic conditions at DH-121, it is likely that the hydrologic responses in the well are similar to those observed in DH-109. Well DH-121 was not instrumented with a waterlevel recorder because of the difficulty of access.

Cottonwood Spring, located approximately $0.25 \mathrm{mi}$ northwest of the Donkey Flats Road crossing of Cottonwood Wash, is a small spring that discharges in the channel of Cottonwood Wash. The spring discharges at an elevation of $5,726 \mathrm{ft}$ above sea level and is near the geologic contact of the Nugget Sandstone and the Carmel Formation.

\section{Groundwater Movement}

Based on water-level measurements in six wells and one spring, altitudes of the potentiometric surface in September 2017 near Red Fleet Reservoir in the Nugget and Frontier Sandstones ranged from 5,578.13 to 5,726.00 ft. The reservoir altitude during this same period was about $5,590 \mathrm{ft}$. The lines on figure 6 show the estimated potentiometric contours (lines of equal groundwater-level altitude) for the Nugget and Frontier Sandstone aquifers. Groundwaterflow gradients observed in September 2017 in the Nugget Sandstone aquifer indicate that groundwater was discharging to Red Fleet Reservoir from the aquifer from the east and west banks of the reservoir. An upward vertical groundwater gradient also was observed in the Nugget Sandstone aquifer as the altitude of the water level in the Red Fleet State Park well, which has an open bottom at a depth of $510 \mathrm{ft}$ below land surface, was 5,645.76 ft compared to 5,640.45 ft in well RF1; well RF1 is $270 \mathrm{ft}$ away and is screened to a depth of $99 \mathrm{ft}$ below land surface (fig. 6).

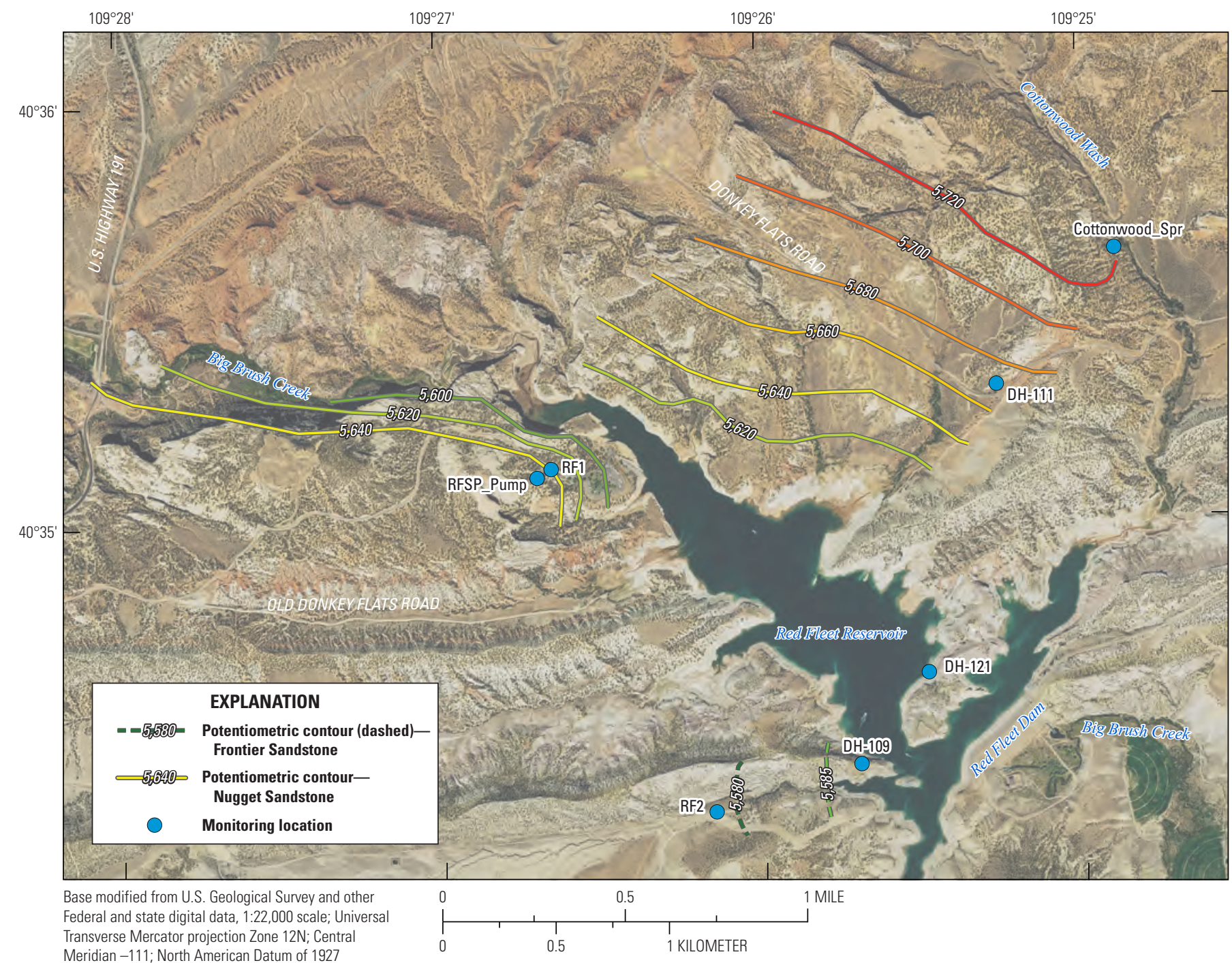

Figure 6. Potentiometric contours of the Nugget and Frontier Sandstone aquifers in September 2017, Red Fleet Reservoir, Utah. 
Groundwater-flow gradients observed in September 2017 in the Frontier Sandstone aquifer indicate that the reservoir was recharging the aquifer on the west bank near Red Fleet Dam. The difference in groundwater altitude in well RF2 and the stage of Red Fleet Reservoir in September 2017 indicates a shallow gradient between the reservoir and the aquifer (fig. 6).

\section{Estimates of Groundwater Recharge and Discharge from Red Fleet Reservoir}

Recharge from and discharge to Red Fleet Reservoir is calculated as the residual with the following water-budget equation (modified from Heilweil and others, 2005):

$$
R=I_{s w}-O_{s w}-O_{p u m p}+P \pm \Delta S-E
$$

where

$$
\begin{aligned}
& R \quad \text { is recharge (acre- } \mathrm{ft} \text { ), } \\
& I_{s w} \quad \text { is surface-water inflow (acre-ft), } \\
& O_{s w}^{s w} \quad \text { is surface-water outflow (acre-ft), } \\
& O_{\text {pump }} \text { is } \quad \text { is pumped outflow (acre-ft), } \\
& P \text { is the amount of precipitation falling directly } \\
& \text { on the reservoir (acre-ft), } \\
& \Delta S \quad \text { is the change in surface-water storage } \\
& \text { (acre-ft), and } \\
& E \quad \text { is evaporation (acre- } \mathrm{ft} \text { ). }
\end{aligned}
$$

The following equation was developed to evaluate the uncertainty for each monthly recharge estimate:

$$
C U=\sum\left[\left(\left|C_{i}\right| / \sum\left|C_{i}\right|\right) * U_{i}\right]
$$

where

$$
\begin{aligned}
& C U \text { is the composite uncertainty fraction } \\
& \text { ( } 2 \text { standard deviation, } 2 \sigma \text { ), } \\
& \left|C_{i}\right| \quad \text { is the absolute value of each component of the } \\
& \text { water budget (acre-ft), } \\
& \Sigma\left|C_{i}\right| \quad \text { is the sum of absolute values of all the water- } \\
& \text { budget components (acre-ft), and } \\
& U_{i} \quad \text { is the uncertainty fraction }(2 \sigma) \text { for each } \\
& \text { individual water-budget component. }
\end{aligned}
$$

The estimated uncertainty fraction is 0.10 (10 percent) for $I_{s w}, O_{s w}$, and $O_{p u m p}$ because these flows are recorded using USGS streamgaging methods, engineered control devices, and calibrated inline flow meters. The estimated uncertainty fraction is 0.10 (10 percent) for $\Delta S$ because changes in surface-water storage are based only on approximate reservoir water-level altitude/volume relations rather than direct measurements. The estimated uncertainty fraction for $P$ is 0.20 (20 percent) because it is an indirect measurement taken on the basis of nearby meteorology station data approximately $10 \mathrm{mi}$ from the study area. The estimated uncertainty fraction is 0.20 ( 20 percent) for $E$, which is based on differences between alternative methods for estimating evaporation at Red Fleet Reservoir and other areas (Heilweil and Susong, 2007; Rosenberry and others, 2007).

The monthly amount of precipitation falling on the reservoir is calculated by multiplying the total monthly precipitation recorded by the Vernal Airport weather station by the average reservoir surface area for that month (based on reservoir water-level altitude to area relations for the reservoir; John Hunting, Uintah Water Conservancy District, written commun., 2016). The precipitation term in equation 1 , however, does not account for precipitation runoff to the reservoir. Summer monsoon precipitation events occasionally produce runoff that reaches Red Fleet Reservoir, primarily through the Cottonwood Wash drainage. The volume of runoff produced by these events is negligible in comparison to the total reservoir water budget.

Monthly water-budget values for Red Fleet Reservoir are shown in table 1. Values are monthly totals, except for reservoir altitude and storage, which are shown for the last day of each month.

\section{Changes in Reservoir Storage}

Changes in reservoir storage were calculated from daily reservoir water-level altitudes reported by the BOR using altitude to volume relations (John Hunting, Uintah Water Conservancy District, written commun., 2016). Since Red Fleet Reservoir began filling in May 1980, surface-water storage increased to a maximum of about 26,000 acre-ft in June 1983. Between 1983 and 2015 surface-water storage in the reservoir seasonally fluctuated from about 15,000 to 26,000 acre- $\mathrm{ft}$ during average to above-average annual precipitation years, but dropped to about 7,000 acre-ft during periods of below-average annual precipitation (table 1).

\section{Reservoir Evaporation}

The McGuinness and Bordne (1972) version of the Jensen-Haise method was selected for calculating evaporation from Red Fleet Reservoir and is based on the relation:

$$
P E T=\left\{\left[\left(\left(0.01 T_{a}\right)-0.37\right)\left(Q_{s}\right)\right] 0.000673\right\} 2.54
$$

where

$$
\begin{array}{cl}
\text { PET } & \text { is potential evaporation, in centimeters per } \\
\text { day, }
\end{array}
$$

The units for PET can be converted to feet per day by multiplying by 0.0328 . 
Monthly evaporation rates were calculated with equation 3 using air temperature and solar radiation from the nearby weather stations (fig. 1). These estimated evaporation rates ranged from 0.00 to 0.85 foot per month from May 1980 through December 2015 (table 1). Multiplying the estimated evaporation rates by the average reservoir surface area yields monthly evaporation losses that ranged from about 0 to 380 acre-ft between May 1980 and December 2015.

\section{Estimates of Groundwater Recharge and Discharge at Red Fleet Reservoir}

Monthly estimates of precipitation $(P)$, evaporation $(E)$, inflow $\left(I_{s w}\right)$, outflows $\left(O_{s w}\right.$ and $\left.O_{p u m p}\right)$, and changes in surfacewater storage $(\Delta S)$ were used in equation 1 to calculate recharge and discharge to and from the Nugget and Frontier Sandstone aquifers beneath Red Fleet Reservoir. Monthly recharge (plus, +) and discharge (minus, -) from May 1980 through December 2015 ranged from about $-2,800$ to 2,200 acre-ft (fig. 7), with $2 \sigma$ composite uncertainties ranging from about 10 to 12 percent of the estimate (table 1). Higher composite uncertainties in the summer reflect the larger, weighted importance of evaporation losses, which have the highest uncertainty.

Annual inflow, dam releases, pumped outflow, evaporation, and groundwater recharge/discharge to and from Red Fleet Reservoir from 1980 through 2015 are shown on figure 3 . During this period, total inflow was about $1,050,000$ acre-ft, with annual inflow ranging from about 12,000 acre-ft in 2002 to 49,000 acre-ft in 1998. From 1980 through 2015, total dam releases were about 993,000 acre-ft, with annual dam releases ranging from about 15,000 acre-ft in 2013 to about 51,000 acre-ft in 1983. Total pumped outflow from 1980 through 2015 was about 42,000 acre-ft, with annual pumped outflow ranging from 0 acre-ft before 1986 to about 3,400 acre-ft in 2007. Total evaporation from 1980 through 2015 was about 52,000 acre-ft, with annual evaporation ranging from about 830 acre-ft in 1980 to about 1,770 acre-ft in 2007. Annual groundwater recharge was greatest in 2007 at about 4,000 acre-ft following a period from early 2001 through early 2005 when the reservoir volume was substantially decreased. Annual groundwater discharge was greatest in 1997 at about 4,900 acre-ft, and total groundwater recharge/discharge to Red Fleet Reservoir from 1980 through 2015 was about 40,000 acre-ft, with a $2 \sigma$ uncertainty of 11,000 acre-ft (fig. 7; table 1).

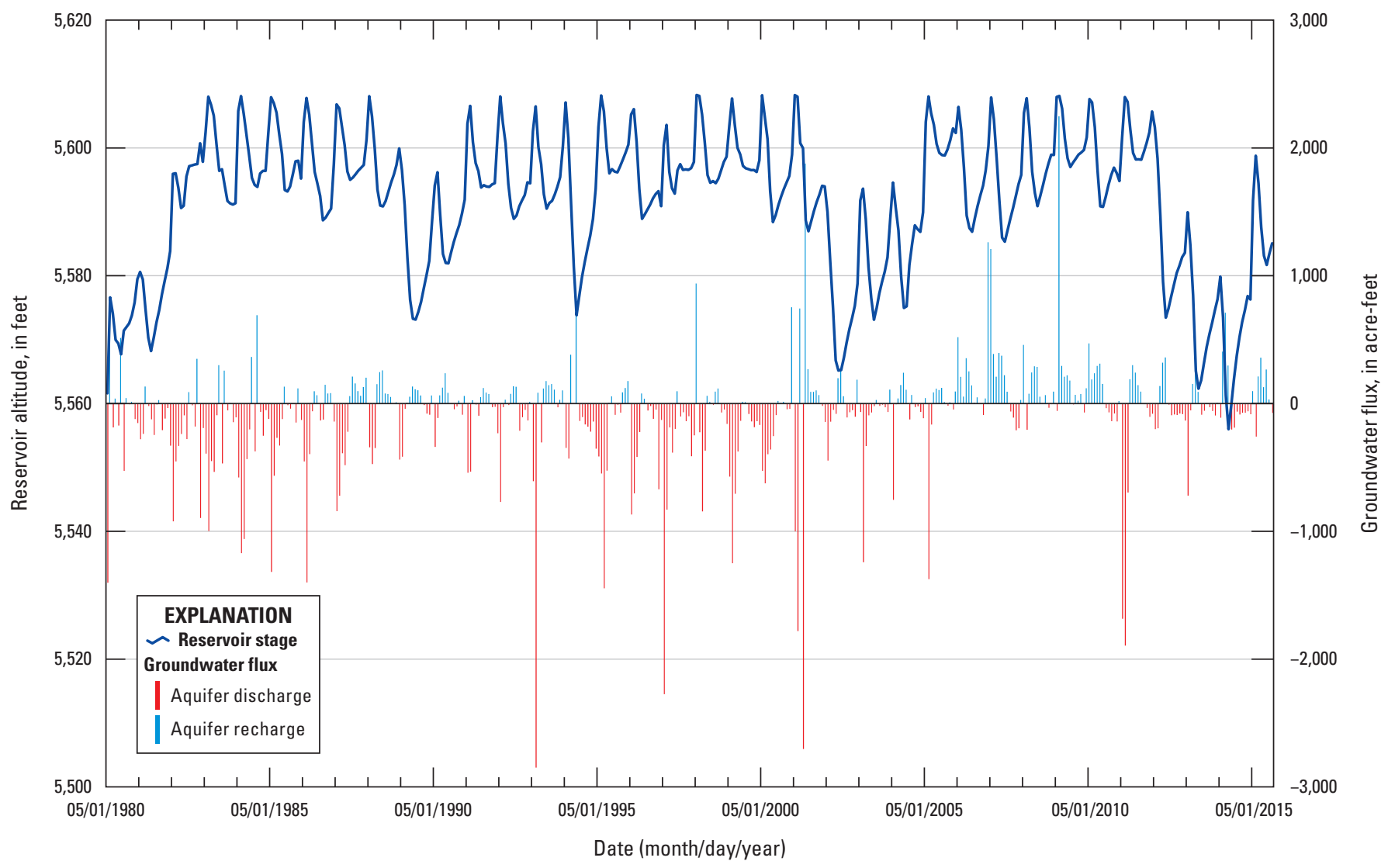

Figure 7. Reservoir stage and calculated groundwater recharge/discharge at Red Fleet Reservoir, Utah, 1980-2015. 


\section{Big Brush Creek Seepage Assessment Upstream of Red Fleet Reservoir}

A seepage study was completed on November 17, 2017, on Big Brush Creek under baseflow conditions to quantify groundwater gains or losses between the Big Brush Creek streamgage (USGS 09261700) and the inflow to Red Fleet Reservoir (fig. 8). Big Brush Creek is fed primarily by Brush Creek Spring approximately 4 mi upstream of the USGS streamgage. The reservoir inflow site, about 1 mi downstream, was chosen to be as close as possible to Red Fleet Reservoir without compromising measurement quality because of slack water. There are no known surface-water inflows, turnouts, or return points throughout the reach.

Two discharge measurements were taken at the downstream inflow site and one at the upstream gaged site.
The measured value at the upstream site was supported by a value computed from the stage-discharge rating model in use at the USGS streamgage. The model was previously developed by calibrating stage to periodic streamflow measurements. Discharge measurements were taken with a SonTek FlowTracker2 handheld Acoustic Doppler Velocimeter using standard USGS methods (Turnipseed and Sauer, 2010). Percent statistical uncertainty values calculated by the FlowTracker2 ADV software are used by the USGS as standard practice for estimating discharge error because of channel conditions and measurement instrumentation. For the full methodology of this calculation see the section "Discharge Uncertainty Calculations Using a SonTek FlowTracker" in SonTek (2009). Mean discharge measured at each location and uncertainty values are shown on figure 9 .

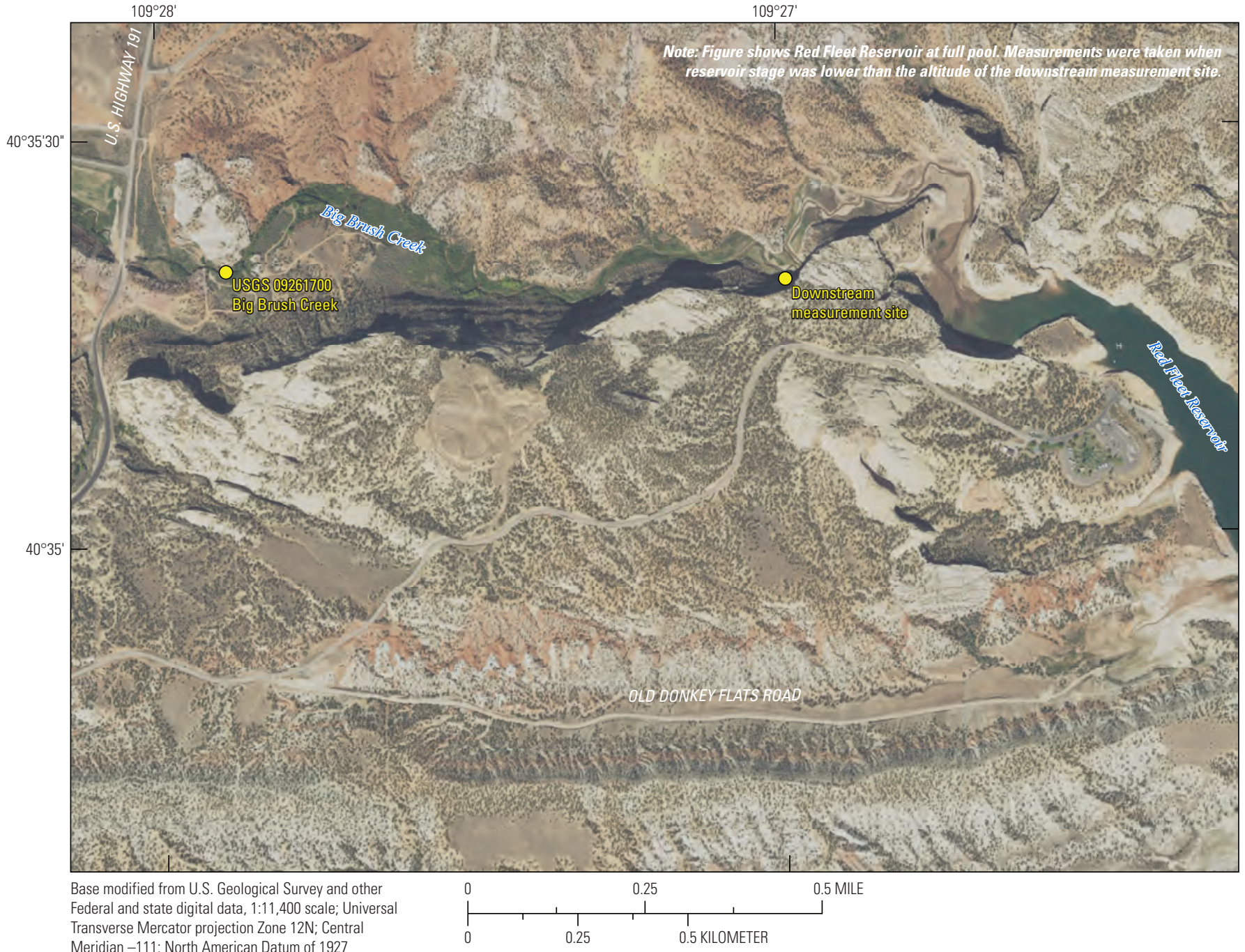

Figure 8. Locations of seepage measurements taken on November 17, 2017, on Big Brush Creek upstream of Red Fleet Reservoir, Utah. 


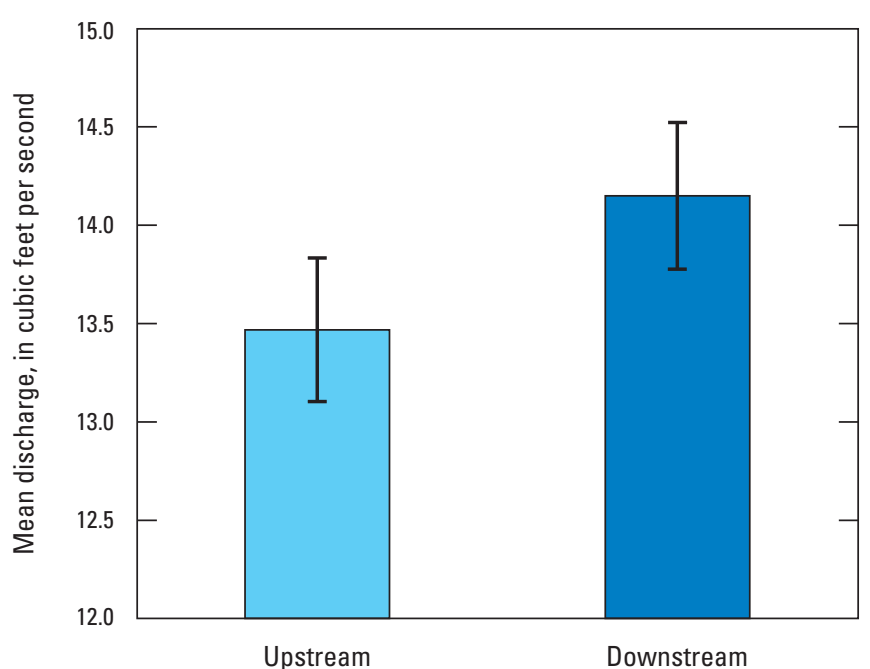

Figure 9. Measured discharge and uncertainty at two stations on Big Brush Creek upstream of Red Fleet Reservoir, Utah.

The measurements spanned a period of relatively steady flow conditions based on the continuous water-stage record obtained from the streamgage (fig. 10). The increase or decrease between measurement sites represents the net seepage interchange between groundwater and surface water. The measurements show an increase in discharge between the upstream and downstream sites of about 0.68 cubic foot per second $\left(\mathrm{ft}^{3} / \mathrm{s}\right)$ or 4.8 percent of the gage streamflow (fig. 10). Calculated seepage over the reach is subject to inherent measurement uncertainty, but is supported by trends in sampled radon concentration observed throughout the stream reach. The increase in flow observed under baseflow conditions indicates a considerable annual groundwater discharge from the Nugget Sandstone aquifer to the stream reach.

\section{Evaluation of Aquifer Properties in the Nugget Sandstone Aquifer near Red Fleet Reservoir}

Regionally, the Nugget Sandstone aquifer is utilized as a good-quality groundwater source for domestic use. To better understand how groundwater flows through the Nugget Sandstone aquifer near Red Fleet Reservoir, a constantdischarge test was completed on the Red Fleet State Park drinking-water supply well (RFSP). This test was used to evaluate aquifer properties in the Nugget Sandstone aquifer near Red Fleet Reservoir to compare to published values in the region. The RFSP well was pumped at an average rate of 11 gallons per minute (gal/min) from March 9, 2017, to March 17, 2017, as part of an 8-day constant-discharge test.

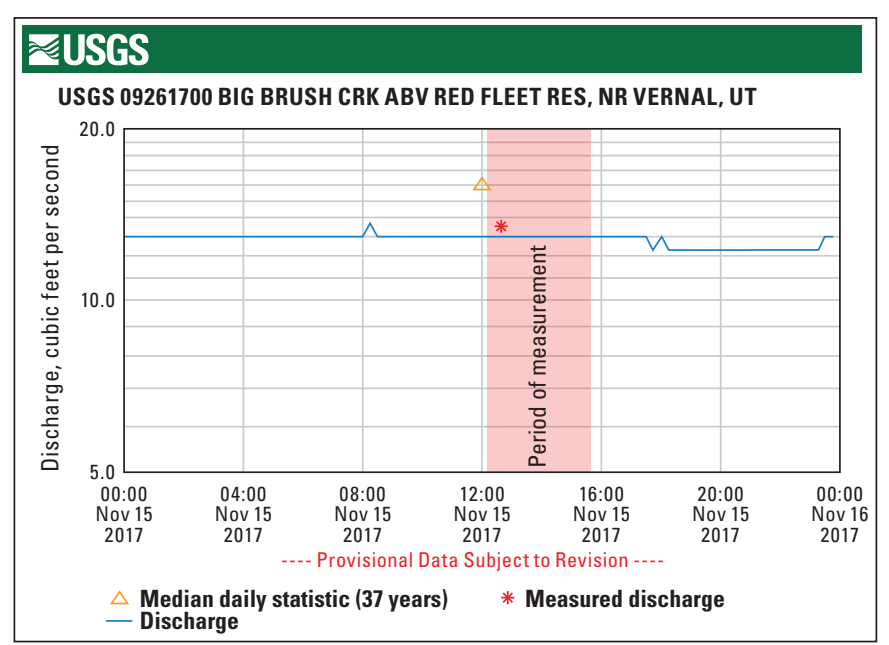

Figure 10. Recorded discharge at the U.S. Geological Survey Big Brush Creek streamgage 09261700 during the November 17, 2017, seepage assessment above Red Fleet Reservoir, Utah.

Water-level data were collected at the pumping well (RFSP) and at monitoring well RF1 for the duration of the test and during a 30-day recovery period so that transmissivity and storage coefficient values of the Nugget Sandstone aquifer could be estimated (fig. 11).

Pumping well RFSP is an 8.375-inch-diameter well completed in an 8.375-inch-diameter borehole. The drillers $\log$ reports that the well only penetrates the Nugget Sandstone aquifer to a total depth of $510 \mathrm{ft}$ below land surface. The well is not screened or perforated and is only open to the aquifer at the base of the well casing. The aquifer is assumed to be unconfined. The static water level before pumping began was $73.05 \mathrm{ft}$ below land surface.

Observation well RF1 is a 2-inch-diameter well completed in a 6-inch-diameter borehole; it was drilled to a depth of $99 \mathrm{ft}$ below land surface and only penetrates the Nugget Sandstone aquifer. The well is screened in the Nugget Sandstone aquifer from 79 to $99 \mathrm{ft}$ below land surface and is $270 \mathrm{ft}$ northeast of the RFSP pumping well. The static water level before pumping began was $53.48 \mathrm{ft}$ below land surface.

\section{Aquifer Test Description and Analysis}

The aquifer test commenced when well RFSP began pumping at 10:35 a.m. on March 9, 2017, and continued for 8 days until 10:35 a.m., March 17, 2017. Discharge from the pumped well ranged from 16.5 to $10.4 \mathrm{gal} / \mathrm{min}$ during the test (fig. 12). Discharge water was routed away from the test site through a 2-inch discharge line to a drainage approximately $700 \mathrm{ft}$ to the southeast (fig. 11). Once pumping had ceased, water levels were allowed to recover for 30 days. During the test and recovery period, water levels in wells RF1 and RFSP were monitored at 5-minute intervals. 


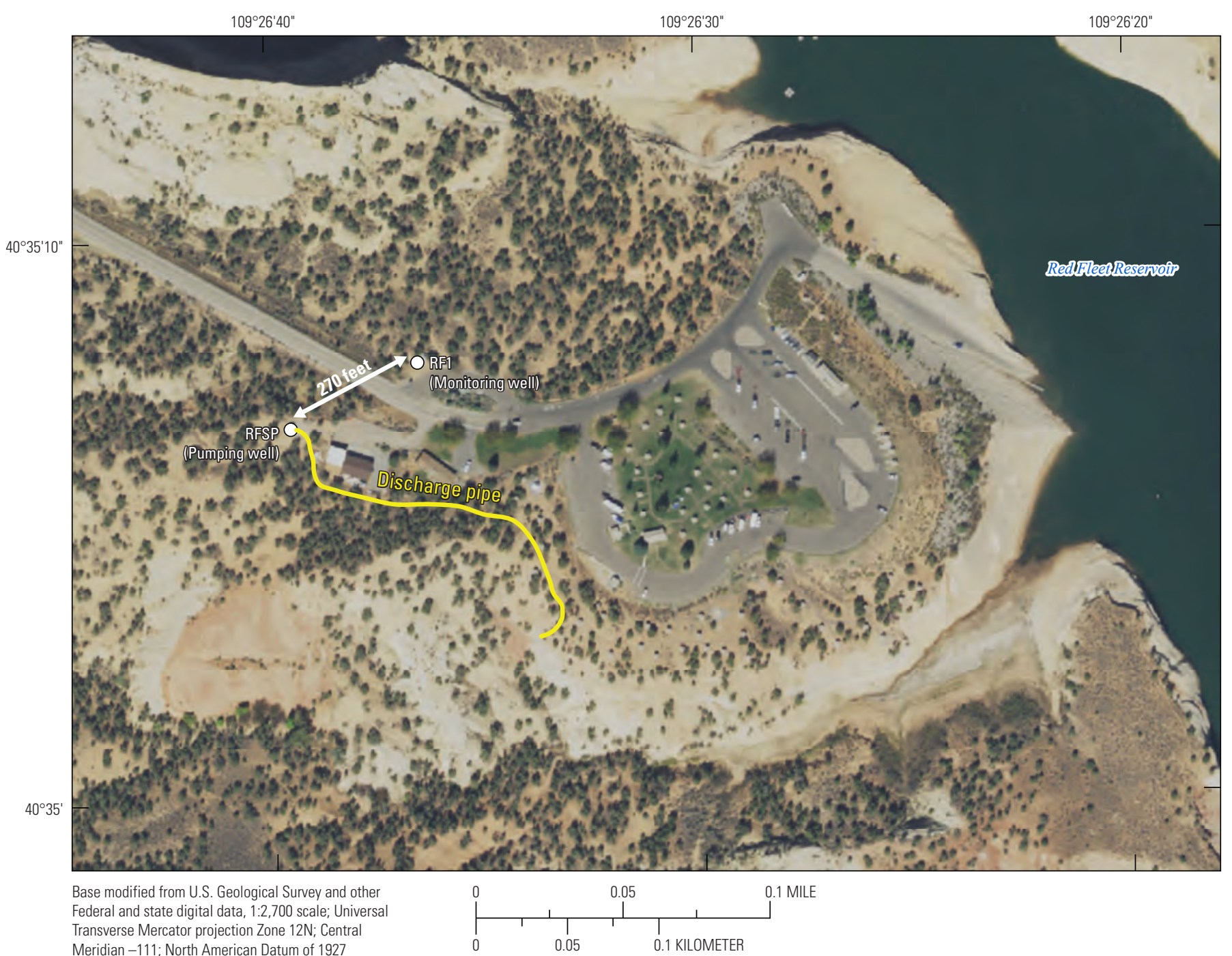

Figure 11. Locations of wells and discharge pipe used during the March 2017 Nugget Sandstone aquifer test at Red Fleet Reservoir, Utah. The Red Fleet State Park well is identified as a "pumping well" and RF1 is identified as a "monitoring well."

An Excel spreadsheet program (SeriesSEE) was used to analyze the data (Halford and others, 2012). A water-level model was constructed to simulate water-level fluctuations observed in well RF1 using data from barometric pressure, a nearby reference well (RF2) that was unaffected by pumping, and a superimposed Theis solution. A series of moving average transforms were applied to the barometric pressure and reference-well data to analyze water-level fluctuations with variable frequencies (fig. 13).
The sum of the transformed data and the superimposed Theis solution created the simulated water level observed on figure 12. The resulting superimposed Theis solution from this water-level model yielded a simulated transmissivity of 660 square feet per day $\left(\mathrm{ft}^{2} / \mathrm{d}\right)$ with a storage coefficient of 0.13 for the Nugget Sandstone aquifer near the RFSP well. The transmissivity and storage coefficient values fall within the range of known values for the Nugget Sandstone in the region. 


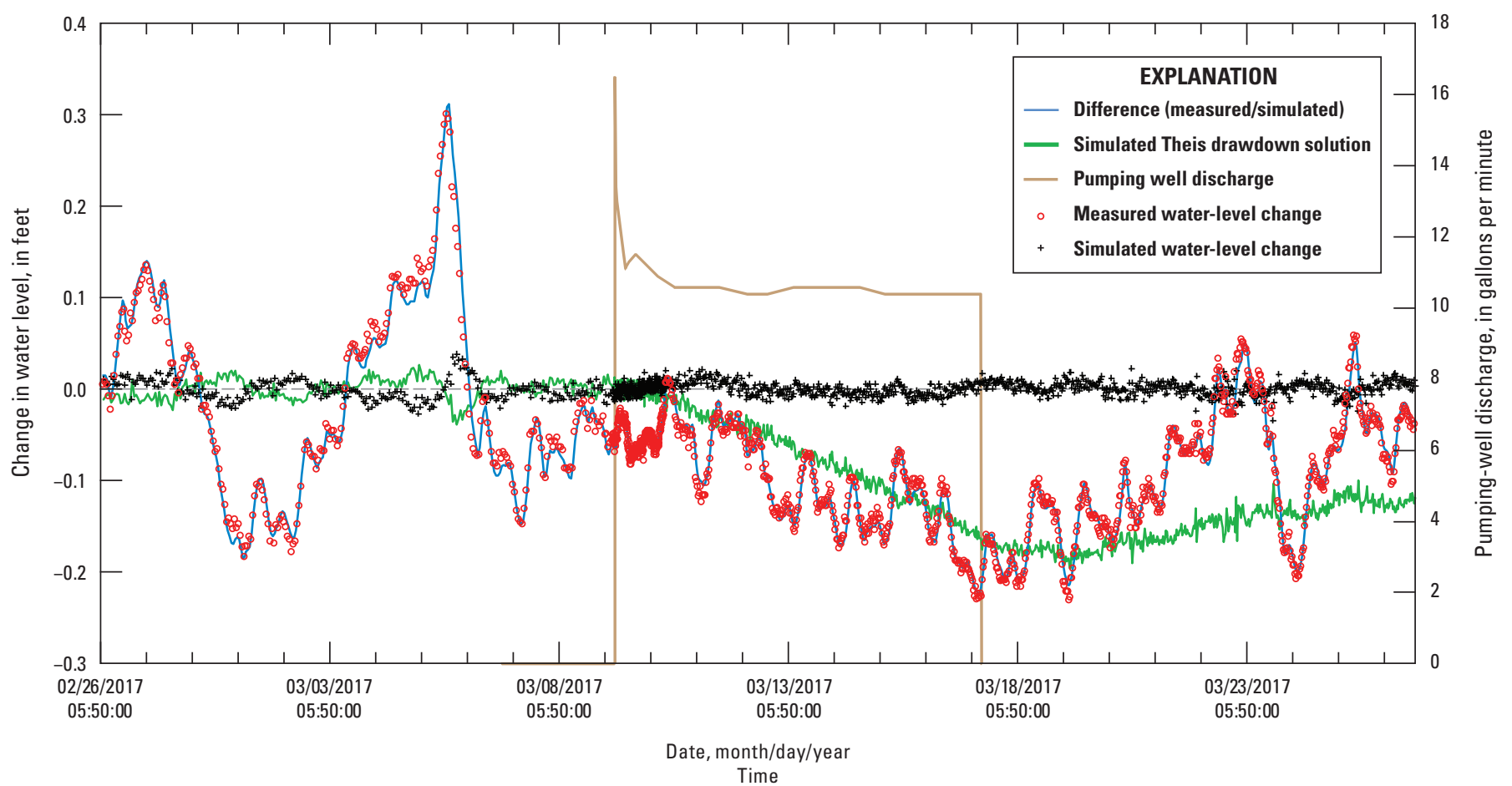

Figure 12. Measured change in water level from observation well RF1 near Red Fleet Reservoir resulting from pumping at the Red Fleet State Park well, and simulated change in water level from a Theis aquifer-response solution, March 2017, Red Fleet Reservoir, Utah. 


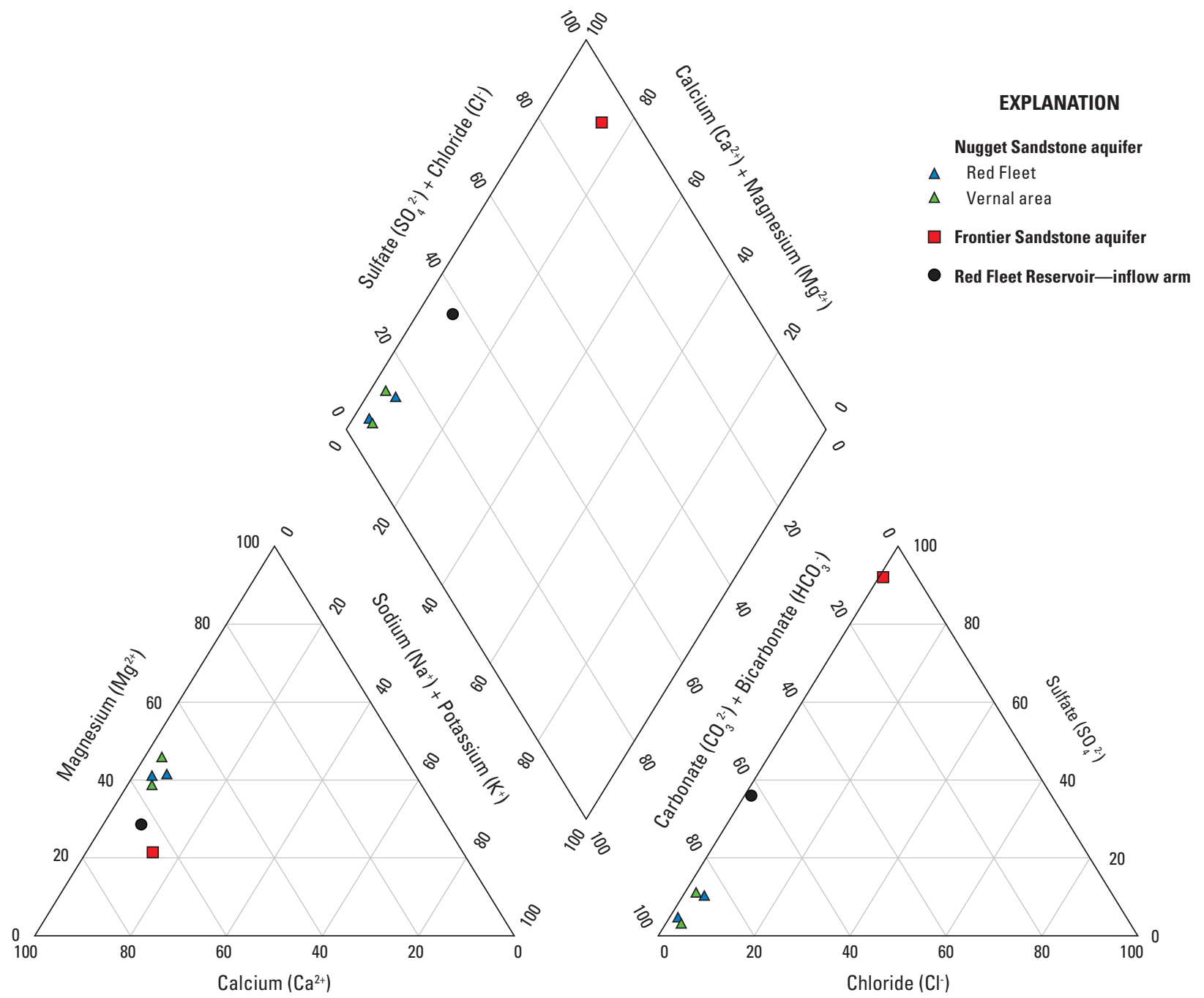

Figure 13. Relative major-ion concentrations for selected wells near Red Fleet Reservoir, Utah. 


\section{Evaluation of Groundwater Geochemical Characteristics in the Nugget and Frontier Sandstones}

Water samples were collected from four sites near and in Red Fleet Reservoir, including two monitoring wells, a culinary well, and surface water from the reservoir. The water samples were analyzed for major ions, nutrients, and selected trace metals to characterize general chemistry and patterns of water quality for a limited area near and in Red Fleet Reservoir. Water samples also were analyzed for several environmental tracers including the radioactive isotope of hydrogen (tritium, ${ }^{3} \mathrm{H}$ ), dissolved noble gases, chlorofluorocarbons, and sulfur hexafluoride. These environmental tracers were used to investigate potential groundwater recharge areas and groundwater ages to provide an understanding of the groundwater interaction that occurs at Red Fleet Reservoir.

Field parameters were measured to provide an on-site characterization of surface-water and groundwater quality. Field water-quality parameters included water temperature, specific conductance, $\mathrm{pH}$, dissolved oxygen, and total dissolved-gas (TDG) pressure. Total dissolved-gas pressure is the combination of the partial pressures of all the dissolved gas in water.

\section{Data Collection Methods}

Field parameters were measured with a multi-parameter sonde placed in the screened interval at the bottom of each 2-inch monitoring well, and in the reservoir at water depths of approximately $3 \mathrm{ft}$. The multi-parameter sonde was too large to enter the 1-inch access port on the Red Fleet State Park drinking-water well. Consequently, field measurements from this well were made on-site with a flow-through chamber connected to a discharge spigot near the well head before the drinking water holding tank.

Laboratory water-quality analyses of surface water from Red Fleet Reservoir and groundwater from the Nugget and Frontier Sandstone aquifers included major and trace dissolved inorganic and organic constituents, ${ }^{3} \mathrm{H}$, and noble and industrial dissolved gases. The major inorganic ions included calcium, magnesium, sodium, potassium, bicarbonate, sulfate, chloride, and nitrate. Trace ions and nutrients included fluoride, bromide, iron, manganese, arsenic, nitrite, ammonia, and orthophosphate. Dissolved organic constituents included dissolved organic carbon. Dissolved gases included chlorofluorocarbons (trichlorofluoromethane, CFC-11; dichlorodifluoromethane, CFC-12; and 1,1,2-trichloro-1,2,2trifluoroethane, $\mathrm{CFC}-113)$, sulfur hexafluoride $\left(\mathrm{SF}_{6}\right)$, and noble gases (helium, He; neon, Ne; argon, Ar; krypton, Kr; and xenon, Xe).
Water-chemistry samples were collected from 2-inch monitoring wells using a Grundfos RediFlo-2 or Geotech SS Geosub sample pump, and production wells were sampled utilizing installed submersible pumps. Before the waterchemistry sample collection from monitoring wells, water was purged from each well until a minimum of three casing volumes were removed and field parameters had stabilized. After purging each well, water was pumped into samples bottles and filtered as necessary.

Samples for major ions and trace ions were filtered with 0.45 -micron disposable filters and collected in clean polyethylene bottles according to procedures described by Wilde and Radtke (1998); samples for cation analysis were preserved with 7.7-normal nitric acid. Tritium samples were collected in 500-milliliter (ml) polyethylene bottles with polyseal caps without head space. The CFC and $\mathrm{SF}_{6}$ samples were collected in 250-ml and 1-liter (L) glass bottles, respectively, according to procedures described at the USGS Reston Groundwater Dating Laboratory (https://water.usgs. gov/lab/). Noble gases were collected with diffusion sampler methods described by Sheldon (2002) and Gardner and Solomon (2009), and with copper-tube methods described by Stute and Schlosser (2000).

Inorganic and organic chemical analyses (major ions, trace ions, and dissolved organic carbon) were analyzed by the USGS at the National Water Quality Laboratory in Denver, Colorado. The CFCs and $\mathrm{SF}_{6}$ were analyzed by the USGS at the Chlorofluorocarbon Laboratory in Reston, Virginia. Tritium and noble gases were analyzed by the University of Utah Dissolved Gas Laboratory using quadrupole and sector-field mass spectrometers; tritium concentrations were determined using the in-growth method (Clarke and others, 1976).

\section{Data Interpretation Methods}

\section{Noble Gases}

Dissolved heavy noble-gas samples (neon-20, ${ }^{20} \mathrm{Ne}$; argon-40, ${ }^{40} \mathrm{Ar}$; krypton- $84{ }^{84} \mathrm{Kr}$; and xenon- $129,{ }^{129} \mathrm{Xe}$ ) were used to determine noble-gas recharge temperatures (NGTs, assumed to equal the temperature of groundwater recharge as it crosses the water table) as an indicator of recharge source (mountain versus reservoir) and groundwater movement. Noble gases dissolved in groundwater primarily are of atmospheric origin, with concentrations being a function of gas solubility (with the possible addition of excess air from dissolution of trapped bubbles) at the temperature, pressure, and salinity conditions when recharge crosses the water table. Variation in the solubility of individual noble gases is such that measured concentrations of multiple noble gases can be used to fit unique models of recharge conditions. Because noble gases are geochemically inert, groundwater NGTs are preserved along the length of a groundwater flow path. 
A complete review of noble gases as groundwater tracers is found in Stute and Schlosser (2000).

For this study, the noble-gas concentrations were interpreted using the closed-system equilibration (CE) model (Aeschbach-Hertig and others, 2000; Kipfer and others, 2002). In addition to recharge temperature, the $\mathrm{CE}$ model also calculates excess air as the dimensionless ratio of the total volume of trapped (moist) air at the pressure and temperature of the free atmosphere to the volume of water (A) and a fractionation factor $(\mathrm{F})$ accounting for partial dissolution of trapped air bubbles. For samples collected in areas of high topographic gradient, the recharge altitude (a proxy for barometric pressure) is an unknown parameter. Because recharge temperature $\left(\mathrm{T}_{\mathrm{r}}\right)$ and recharge altitude are correlated, a range of NGTs (assumed to equal $\mathrm{T}_{\mathrm{r}}$ ) was calculated for each sample with the most likely NGT determined by the intersection of calculated NGT with the temperature lapse rate, a method described by Manning and Solomon (2003) and Manning (2011). The range of possible NGTs was based on the minimum recharge altitude, typically that of the sample site and the maximum possible recharge altitude, which was estimated to be $10,000 \mathrm{ft}$ for all samples based on the elevation of high-altitude plateaus and lakes in the Uinta Mountains where recharge is most likely to occur. Temperature lapse rate and change in temperature as a function of altitude were determined at points along south to north transects starting at the study area and based on 30-year mean annual air temperature (PRISM Climate Group, 2012) and the National Elevation Dataset (U.S. Geological Survey, 2017). A watertable lapse rate was estimated based on general observations of a $+3{ }^{\circ} \mathrm{C}$ difference between air and water-table temperatures (Manning and Solomon, 2003). The recharge parameters (NGT, A, and F) were evaluated across the range of recharge altitudes with a standard Newton inversion technique to minimize the error-weighted misfit $\left(\chi^{2}\right)$ between measured and modeled dissolved-gas concentrations (Aeschbach-Hertig and others, 2000; Manning and Solomon, 2003). An $\chi^{2}$ probability threshold of 3.84, based on four measured gases and three recharge parameters (P greater than 0.05), was used to define good model fits for NGT, A, and F. Because of noble-gas measurement precision, uncertainty in NGTs is generally 0.5-1.5 ${ }^{\circ} \mathrm{C}$ (Manning and Solomon, 2003; Manning, 2009; Masbruch and others, 2012).

\section{Tritium and Helium Isotopes}

Tritium and helium isotopes were used in this study to evaluate the age of groundwater and reservoir samples. Tritium is a radioactive isotope of hydrogen with a half-life of 12.32 years (Lucas and Unterweger, 2000) that decays to tritiogenic helium-3 $\left({ }^{3} \mathrm{He}_{\text {trit }}\right)$. Tritium is present in water as part of the water molecule, whereas its decay product $\left({ }^{3} \mathrm{He}_{\text {trit }}\right)$ is a component of the total helium budget dissolved in water. During the 1950s and 1960s, large amounts of ${ }^{3} \mathrm{H}$ were released into the atmosphere and introduced into the hydrologic cycle by above-ground thermonuclear weapons testing. As a result, ${ }^{3} \mathrm{H}$ concentrations in precipitation in the northern hemisphere during 1963-64 peaked at three orders of magnitude above natural concentrations (Michel, 1989).

Concentrations of ${ }^{3} \mathrm{H}$ and ${ }^{3} \mathrm{He}_{\text {trit }}$ can be used to determine the apparent age of groundwater that is less than about 60 years old. These ages are referred to as "apparent" as the calculation assumes plug flow along a single flow path (for example, piston flow) and can differ from the true mean age of the sample if it contains a mixture of water of different ages. Mixtures of modern (post-mid-1950s recharge) and pre-modern (pre-mid-1950s recharge) water typically have apparent ${ }^{3} \mathrm{H} /{ }^{3} \mathrm{He}_{\text {trit }}$ ages that represent the age of the young fraction of the sample because dilution with pre-modern water will leave the ratio of ${ }^{3} \mathrm{H}$ to ${ }^{3} \mathrm{He}_{\text {trit }}$ virtually unchanged. Further details of this groundwater dating method are presented in Solomon and Cook (2000).

Although ${ }^{3} \mathrm{H}$ in modern precipitation was not measured during this study, recharge after 2012 is assumed to contain 6-11 tritium units (TU) as indicated by empirical relationships derived from measured ${ }^{3} \mathrm{H}$ across a range of latitude and longitude (Michel, 1989). In a sample of pre-modern groundwater, ${ }^{3} \mathrm{H}$ would have decayed from background "prebomb" concentrations of about $8 \mathrm{TU}$ to less than $0.3 \mathrm{TU}$, which is approaching the analytical detection limit. Samples collected during this study having concentrations of $0.4 \mathrm{TU}$ or less and accounting for a typical analytical uncertainty of $0.1 \mathrm{TU}$, were interpreted to contain no modern water. Apparent ${ }^{3} \mathrm{H}^{3} \mathrm{He}_{\text {trit }}$ ages were computed for samples that had concentrations of more than $0.4 \mathrm{TU}$.

In addition to ${ }^{3} \mathrm{He}$ derived from ${ }^{3} \mathrm{H}$ decay, groundwater also accumulates dissolved helium because it is produced from the radioactive decay of naturally occurring uranium- and thorium-series elements in aquifer solids (crustal $\mathrm{He}$ ) and from the upward advection and (or) diffusion of primordial helium from the mantle (mantle $\mathrm{He}$ ). Crustal- and mantle-sourced $\mathrm{He}$ are collectively referred to as "terrigenic $\mathrm{He}$ " $\left(\mathrm{He}_{\text {terr }}\right.$; Solomon, 2000). Crustal- and mantle-sourced $\mathrm{He}$ are distinguishable by their relative abundance of ${ }^{3} \mathrm{He}$ and ${ }^{4} \mathrm{He}$ (helium-4) isotopes, and because $\mathrm{He}_{\text {terr }}$ concentrations generally increase with increasing residence time. Dissolved terrigenic helium-4 $\left({ }^{4} \mathrm{He}_{\text {terr }}\right)$ concentrations have been used as a semiquantitative tool for dating groundwater with ages from 1,000 to more than 1,000,000 years (Mazor and Bosch, 1992; Solomon, 2000). No attempts were made to accurately date groundwater in this study using ${ }^{4} \mathrm{He}_{\text {terr }}$ because crustal $\mathrm{He}_{\text {terr }}$ production rates are highly variable and substantial additional data would have been required to constrain these rates within the study area. Even without precise knowledge of local ${ }^{4} \mathrm{He}$ production rates, ${ }^{4} \mathrm{He}$ concentrations in excess of atmospheric solubility are useful as qualitative measures of groundwater age. 


\section{Lumped Parameter Modeling}

\section{Categorical Tritium Age}

Measured tritium values can be used to categorically define the age of groundwater by correcting the estimated annual tritium concentration of local precipitation for the radioactive decay of tritium, which has a half-life of 12.32 years (Lucas and Unterweger, 2000). For this study, the categories were defined as pre-modern (pre-1953 recharge) with measured ${ }^{3} \mathrm{H}$ concentrations less than $0.4 \mathrm{TU}$, modern (post-1953 recharge) with ${ }^{3} \mathrm{H}$ concentrations of greater than $3.5 \mathrm{TU}$, and a mixture of pre-modern and modern water with ${ }^{3} \mathrm{H}$ concentrations between these two values. The categorical age is useful in assigning a generalized age and in identification of outlier tracers because ${ }^{3} \mathrm{H}$ is the least susceptible to contamination or other in situ alterations.

\section{Groundwater Age}

An estimated mean age and age distribution, describing the relative contribution of various flow paths, were determined for each sample using a modified version of TracerLPM (Jurgens and others, 2012; Bryant Jurgens, U.S. Geological Survey, written commun., 2013) and a local hydrogeologic conceptualization. The lumped parameter modeling (LPM) approach assumes steady-state groundwater flow and conservative tracer behavior (with the exception of radioactive decay). The selected conceptual age distribution for a given sample is mathematically convoluted with the measured atmospheric tracer concentrations (input functions) to calculate tracer concentrations for any given mean age. The mean age and age-distribution parameters are optimized to match the measured and simulated tracer concentrations. Selection of the most suitable LPM is based on multiple lines of evidence including conceptual hydrogeology, tracer concentrations, and LPM results, and is an iterative process. Geologic cross sections and lithologic well logs were used to develop a conceptual model of the hydrogeology. The conceptual hydrogeology provides context and physical constraints on the selection of a suitable distribution (see "Jurgens and others, 2012" for conceptual flow-path diagrams). For this study, the dispersion model (DM) with a small dispersion parameter of 0.001 (inverse of the Peclet number; Jurgens and others, 2012) was used in lieu of the piston-flow model, which does not account for hydrodynamic dispersion along flow paths in real groundwater-flow systems. Use of a DM brings the conceptualized flow system represented by the LPM more in line with known physical processes. Measured tracer concentrations are used to select tracers for LPM modeling and refine age-distribution selection. Tracer cross-plots are a graphical representation of the convolution of two tracer input functions for a given age distribution and are used to check consistency between tracer pairs and identify anomalous tracer concentrations which could indicate tracer contamination or biodegradation. Relative merit of the optimized mean age and age distribution are assessed by the number of fitted tracers, the sum of chisquared statistic $\left(\chi^{2}\right)$, tracer cross-plots, objective judgment regarding distribution model parameters, and tracer selection based on the conceptual hydrogeology and other nearby samples.

\section{Water-Quality Results}

\section{Major Ions, Nutrients, and Trace Metals}

Water-quality results from the Nugget and the Frontier Sandstones indicated two distinct chemical water types. The Nugget Sandstone aquifer near Red Fleet Reservoir can be characterized as calcium-magnesium-bicarbonate water with dissolved-solids concentrations that ranged from 135 to 240 milligrams per liter (mg/L; fig. 13; table 2). Concentrations of trace metals sampled including arsenic, selenium, and uranium, were all low with ranges of $0.05-$ 0.07 micrograms per liter $(\mu \mathrm{g} / \mathrm{L})$ for arsenic, $0.46-2.11 \mu \mathrm{g} / \mathrm{L}$ for selenium, and $0.71-8.35 \mu \mathrm{g} / \mathrm{L}$ for uranium. All of the concentrations for trace metals and nutrients in Nugget Sandstone wells sampled near Red Fleet Reservoir were below the Environmental Protection Agency (EPA) maximum contaminant levels. Overall water quality of the wells sampled in the Nugget Sandstone indicates that the aquifer is a good source of drinking water. Geochemical results for two wells west of the study area (fig. 1), but within the Vernal, Utah, region are included in figure 13 and table 2 for comparison to known Nugget Sandstone aquifer geochemistry outside the influence of a nearby reservoir. Water from Vernal area wells screened in the Nugget Sandstone was very similar to water sampled from wells near Red Fleet Reservoir, indicating that the reservoir has limited interaction with the Nugget Sandstone aquifer.

A sample from well RF2 was used to characterize and evaluate water quality in the Frontier Sandstone aquifer near Red Fleet Reservoir (fig. 13; table 2). This sample was characterized as a calcium-magnesium-sulfate water with a dissolved-solids concentration of $2,150 \mathrm{mg} / \mathrm{L}$. The trace metals sampled included arsenic at $9.42 \mu \mathrm{g} / \mathrm{L}$, selenium at $15.2 \mu \mathrm{g} / \mathrm{L}$, and uranium at $1.33 \mu \mathrm{g} / \mathrm{L}$; all were below EPA maximum contaminant levels. The nitrate and nitrite concentrations were well below EPA maximum contaminant levels for drinking water of 10 and $1 \mathrm{mg} / \mathrm{L}$, respectively. The elevated selenium concentration in well RF2 was likely derived from the overlying Mancos Shale, a known reservoir of selenium in the region. Overall water quality in well RF2, which represents the upper part of the Frontier Sandstone aquifer, was poor because of the high dissolved-solids concentration. 
Table 2. Field measurements and major ions, selected trace elements, and nutrient concentrations in groundwater and surface water from selected sites near Red Fleet Reservoir, Utah.

[Sample sites are plotted on figure 2. Abbreviations: USGS, U.S. Geological Survey; ${ }^{\circ} \mathrm{C}$, degrees Celsius; $\mu \mathrm{S} / \mathrm{cm}$, microsiemens per centimeter at 25 degrees Celsius; mg/L, milligrams per liter; $\mathrm{Cl}$ :Br, chlorideto-bromide ratio; $\mu \mathrm{g} / \mathrm{L}$, micrograms per liter; RF1 and RF2 are wells; RFSP, Red Fleet State Park; —, no data available; <, less than]

\begin{tabular}{|c|c|c|c|c|c|c|c|c|c|c|c|c|c|c|c|}
\hline Site name & $\begin{array}{l}\text { USGS site } \\
\text { identifier }\end{array}$ & Date & $\begin{array}{c}\text { Tempera- } \\
\text { ture } \\
\left({ }^{\circ} \mathrm{C}\right)\end{array}$ & $\begin{array}{c}\text { Specific } \\
\text { conduc- } \\
\text { tance } \\
(\mu \mathrm{S} / \mathrm{cm})\end{array}$ & \multicolumn{2}{|c|}{ 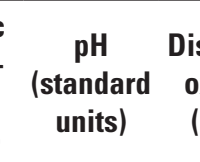 } & $\begin{array}{l}\text { Oissolved } \\
\text { oxygen } \\
\text { (mg/L) }\end{array}$ & $\begin{array}{l}\text { Total dis- } \\
\text { solved } \\
\text { solids } \\
\text { (mg/L) }\end{array}$ & $\begin{array}{c}\text { Calcium } \\
\text { (mg/L as } \\
\text { Ca) }\end{array}$ & $\begin{array}{l}\text { Magne- } \\
\text { sium } \\
\text { (mg/L as } \\
\mathrm{Mg})\end{array}$ & $\begin{array}{c}\text { Sodium } \\
\text { (mg/L as } \\
\mathrm{Na})\end{array}$ & $\begin{array}{c}\text { Potas- } \\
\text { sium } \\
\text { (mg/L as } \\
\mathrm{K})\end{array}$ & $\begin{array}{c}\text { Alkalinity } \\
(\mathrm{mg} / \mathrm{L} \text { as } \\
\left.\mathrm{CaCO}_{3}\right)\end{array}$ & 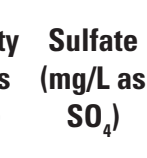 & $\begin{array}{cc}\text { e } & \text { Chloride } \\
\text { as } & \text { (mg/L as } \\
\mathrm{CI})\end{array}$ \\
\hline RF1 & 403508109263901 & $10 / 13 / 2016$ & 10.9 & 302 & 8 & & 9.1 & 168 & 35.1 & 16.7 & 4.26 & 1.67 & 142 & 12.9 & 4.66 \\
\hline RFSP & 403506109264201 & $10 / 13 / 2016$ & 11.0 & 228 & 8 & .3 & 8.9 & 135 & 28.8 & 12.8 & 1.79 & 1.13 & 120 & & 1.58 \\
\hline (D-3-21)20bac-1 & 403254109352401 & $10 / 24 / 2014$ & 10.9 & 247 & 8 & .0 & - & 139 & 31.3 & 12.7 & 2.79 & 0.92 & 127 & & 2.81 \\
\hline$(\mathrm{D}-3-21) 20 \mathrm{ccc}-1$ & 403215109354501 & $9 / 17 / 2013$ & 11.4 & 466 & & & - & 240 & 56.0 & 30.2 & 3.35 & 2.63 & 248 & 24.4 & 4.26 \\
\hline RF2 & 403418109260901 & $10 / 12 / 2016$ & 11.0 & 2,810 & 6 & .9 & 0.0 & 2,150 & 391 & 76.9 & 95.7 & 5.39 & 120 & 1,240 & 14.3 \\
\hline $\begin{array}{r}\text { Red Fl } \\
\text { inflo }\end{array}$ & 403451109260001 & $10 / 13 / 2016$ & 15.0 & 270 & 8 & & 7.2 & 172 & 38.1 & 10.2 & 4.91 & 1.09 & 96.4 & 43.8 & 1.64 \\
\hline Site & $\begin{array}{l}\text { USGS site } \\
\text { identifier }\end{array}$ & Date & $\begin{array}{c}\text { Fluoride } \\
\text { (mg/L } \\
\text { as F) }\end{array}$ & $\begin{array}{c}\text { Bromide } \\
\text { (mg/L as } \\
\mathrm{Br})\end{array}$ & $\mathrm{Cl}: \mathrm{Br}$ & $\begin{array}{c}\text { Silica } \\
\text { (mg/L as } \\
\left.\mathrm{SiO}_{2}\right)\end{array}$ & $\begin{array}{c}\text { Arsenic } \\
\text { is } \mu \mathrm{g} / \mathrm{L} \text { as } \\
\text { As) }\end{array}$ & $\begin{array}{cc}c & \text { Iron } \\
s & (\mu \mathrm{g} / \mathrm{L} \text { as } \\
\mathrm{Fe})\end{array}$ & $\begin{array}{c}\text { Manga- } \\
\text { nese } \\
(\mu \mathrm{g} / \mathrm{L} \text { as } \\
\mathrm{Mn})\end{array}$ & $\begin{array}{c}\begin{array}{c}\text { Sele- } \\
\text { nium } \\
(\mu \mathrm{g} / \mathrm{L} \text { as }\end{array} \\
\mathrm{Se})\end{array}$ & $\begin{array}{l}\text { Uranium } \\
(\mu \mathrm{g} / \mathrm{L} \text { as } \\
\mathrm{U})\end{array}$ & $\begin{array}{c}\text { Nitrogen } \\
\text { (nitrite + } \\
\text { nitrate) } \\
\text { (mg/L as } \\
\mathrm{N})\end{array}$ & $\begin{array}{c}\text { Nitrogen, } N \\
\text { nitrite a } \\
\text { (mg/L as ( } \\
\mathrm{N})\end{array}$ & $\begin{array}{l}\text { Nitrogen, } P \\
\text { ammonia } \\
(\mathrm{mg} / \mathrm{L} \text { as } \\
\mathrm{N} \text { ) }\end{array}$ & $\begin{array}{l}\text { Phosphorous } \\
\text { (orthophos- } \\
\text { phate) } \\
\text { (mg/L as P) }\end{array}$ \\
\hline RF1 & 403508109263901 & $10 / 13 / 2016$ & 0.17 & 0.048 & 97.1 & 9.83 & 0.07 & 13.1 & 0.47 & 2.11 & 1.14 & 0.94 & $<0.001$ & $<0.01$ & $<0.004$ \\
\hline RFSP & 403506109264201 & $10 / 13 / 2016$ & 0.11 & 0.015 & 105.3 & 8.85 & 0.05 & $<5$ & $<0.20$ & 1.05 & 0.71 & 0.50 & $<0.001$ & $<0.01$ & $<0.004$ \\
\hline (D-3-21)20bac-1 & 403254109352401 & $10 / 24 / 2014$ & 0.14 & - & - & 10.1 & $<0.10$ & $<4$ & $<0.20$ & 0.46 & 1.58 & 0.83 & - & $<0.01$ & $<0.004$ \\
\hline$(\mathrm{D}-3-21) 20 \mathrm{ccc}-1$ & 403215109354501 & $9 / 17 / 2013$ & 0.19 & - & - & 7.41 & 0.20 & 235 & 9.95 & 0.69 & 8.35 & $<0.01$ & - & - & $<0.004$ \\
\hline RF2 & 403418109260901 & $10 / 12 / 2016$ & 0.58 & 0.113 & 126.5 & 40.1 & 9.42 & 10,000 & 321 & 15.2 & 1.33 & $<0.04$ & $<0.001$ & 0.31 & 0.021 \\
\hline $\begin{array}{l}\text { RedFleet Reservoir } \\
\text { inflow arm }\end{array}$ & 403451109260001 & $10 / 13 / 2016$ & 0.10 & 0.010 & 164.0 & 5.69 & 0.97 & 9.6 & 1.07 & 0.51 & 1.03 & $<0.04$ & 0.002 & $<0.01$ & $<0.004$ \\
\hline
\end{tabular}


Red Fleet Reservoir water sampled from the inflow arm was characterized as a calcium-sulfate-bicarbonate water with a dissolved-solids concentration of $172 \mathrm{mg} / \mathrm{L}$ (fig. 13; table 2). The reservoir water did have higher concentrations of sulfate than surrounding Nugget Sandstone aquifer water, which was likely the result of dissolution of sulfatebearing minerals upstream of Red Fleet Reservoir in the Big Brush Creek drainage. The trace metals sampled included arsenic at $0.97 \mu \mathrm{g} / \mathrm{L}$, selenium at $0.51 \mu \mathrm{g} / \mathrm{L}$, and uranium at $1.03 \mu \mathrm{g} / \mathrm{L}$; all were below EPA maximum contaminant levels. The nitrate and nitrite concentrations were well below EPA maximum contaminant levels for drinking water of 10 and $1 \mathrm{mg} / \mathrm{L}$, respectively.

\section{Environmental Tracer Results}

\section{Noble-Gas Analysis}

Noble-gas samples were collected at three groundwater wells with measured concentrations of $\mathrm{Ne}, \mathrm{Ar}, \mathrm{Kr}$, and $\mathrm{Xe}$ being well explained by the CE model ( $\chi^{2}$ fits less than 0.5 for all samples) that indicated low amounts of excess air that was moderately fractionated (table 3; fig. 1). A full range of possible NGTs were calculated for each site (fig. 14), with most likely NGTs determined by the intersection of water table and air lapse rates with the modeled NGT lapse rate. Air-temperature lapse rates were used for two sites (RF1 and RFSP) because calculated NGTs did not intersect estimated water-table temperatures. This indicates that the geothermal gradient does not have a strong effect on water-table temperature in the recharge zone for these two sites, possibly as a result of relatively rapid recharge (see "Manning and Solomon, 2003" for further discussion of the relation between mean annual air temperature and recharge temperature). The cool NGTs of 0 and $2.36{ }^{\circ} \mathrm{C}$ for RFSP and RF1, respectively, and corresponding recharge elevations (table 3; fig. 14), indicate that high-elevation snowmelt is the primary recharge source. The warmer NGT of $7.51^{\circ} \mathrm{C}$ at RF2 indicates a larger proportion of low-elevation recharge, with warmer recharge temperatures, was being captured at the well. As shown by hydraulic gradients, groundwater chemistry, and estimated ages, the warmer low-elevation recharge was not likely to be recharge from the reservoir, but rather lower elevation mountain recharge.

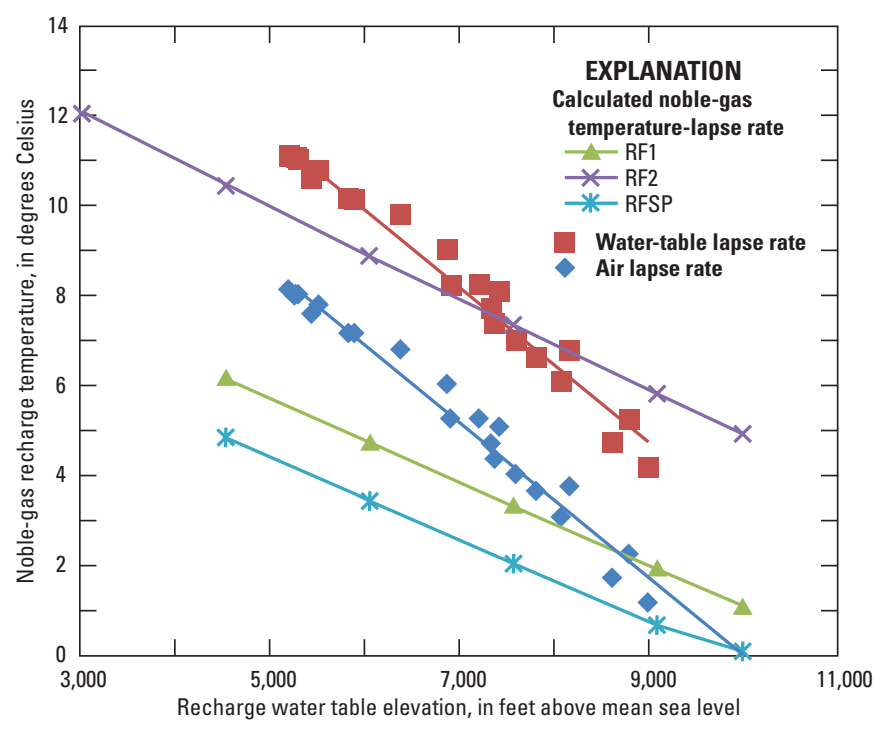

Figure 14. Modeled noble-gas recharge temperature and elevation for groundwater sampled near Red Fleet Reservoir, Utah.

Table 3. Noble-gas model results from samples collected at Red Fleet Reservoir, Utah.

[R/Ra, ratio of helium-3 to helium-4 (He-4) measured in the sample to the atmosphere (Atmo,); ccSTP/g, cubic centimeters at standard temperature and pressure per gram of water; ${ }^{\circ} \mathrm{C}$, degrees Celsius; atm, atmospheres; $\mathrm{F}$, fractionation factor; $\Delta \mathrm{Ne}$, percent $(\%)$ difference between measured and modeled neon concentration; $\chi 2$, chi-squared model fit; RFSP, Red Fleet State Park; —, no data available; Ne, neon; Ar, argon; Kr, krypton; Xe, xenon; RF1 and RF2 are wells]

\begin{tabular}{lcccccccccc} 
Site & $\begin{array}{c}\text { Elevation } \\
\text { (feet) }\end{array}$ & $\begin{array}{c}\text { R/Ra } \\
\text { (unitless) }\end{array}$ & $\begin{array}{c}\text { He-4 } \\
\text { (ccSTP/g) }\end{array}$ & $\begin{array}{c}\text { NGT } \\
\left.\text { ( }{ }^{\circ} \mathbf{C}\right)\end{array}$ & $\begin{array}{c}\text { Excess air } \\
\text { (ccSTP/g) }\end{array}$ & $\begin{array}{c}\text { Atmo. } \\
\text { pressure } \\
\text { (atm) }\end{array}$ & $\begin{array}{c}\mathbf{F} \\
\text { (unitless) }\end{array}$ & $\begin{array}{c}\Delta \text { Ne } \\
\text { (\%) }\end{array}$ & $\begin{array}{c}\text { Gases } \\
\text { modeled }\end{array}$ \\
\hline RFSP & 3,300 & 1.01 & $4.60156 \mathrm{E}-08$ & 0 & 0.02 & - & 0.66 & 0.0004 & 0.48 & $\mathrm{Ne}, \mathrm{Ar}, \mathrm{Kr}, \mathrm{Xe}$ \\
RF1 & 2,850 & 1.02 & $5.82296 \mathrm{E}-06$ & 2.36 & 0.03 & 0.71 & 0.59 & -0.0004 & 0.17 & $\mathrm{Ne}, \mathrm{Ar}, \mathrm{Kr}, \mathrm{Xe}$ \\
RF2 & 2,450 & 0.79 & $8.36451 \mathrm{E}-06$ & 7.51 & 0.06 & 0.74 & 0.51 & 0.0012 & 0.36 & $\mathrm{Ne}, \mathrm{Ar}, \mathrm{Kr}, \mathrm{Xe}$ \\
\hline
\end{tabular}




\section{Age Tracers and Mean Age}

Measured ${ }^{3} \mathrm{H}$ ranged from 0.2 to $7.9 \mathrm{TU}$ with corresponding categorical ${ }^{3} \mathrm{H}$ ages that varied from modern to pre-modern, and calculated ${ }^{3} \mathrm{He}_{\text {trit }}$ values that ranged from 0 to $3 \mathrm{TU}$ (table 4). Tracer cross-plots show ${ }^{3} \mathrm{He}_{\text {trit }}$ values were lower than expected, based on known atmospheric tracer histories, even for a mixture of modern and pre-modern water (fig. 15). Lower than expected ${ }^{3} \mathrm{He}_{\text {trit }}$ was possibly a result of error in the helium balance as indicated by calculated values of initial ${ }^{3} \mathrm{H}$ (sum of ${ }^{3} \mathrm{H}$ and ${ }^{3} \mathrm{He}_{\text {trit }}$ ). Initial ${ }^{3} \mathrm{H}$ should be equal to or greater than the background ${ }^{3} \mathrm{H}$ of $8 \mathrm{TU}$ from cosmogenic production. Initial ${ }^{3} \mathrm{H}$ less than $8 \mathrm{TU}$ is one indication that ${ }^{3} \mathrm{He}_{\text {trit }}$ is not entirely reliable and should be cautiously interpreted. Measured CFC concentrations were internally inconsistent with disagreement between the CFCs (CFC-11, $-12,-113)$ and historical record of measured atmospheric concentrations, indicating contamination or degradation, making age determinations based on CFCs unreliable; thus, CFC concentrations are not reported here.

Estimated mean ages, calculated based on ${ }^{3} \mathrm{H}$ only and the selected age distributions, ranged from 25 to 73 years (table 4). The exponential mixing model was most appropriate for the reservoir because it represents mixing of the full range of flow-path ages. Samples from wells were best represented by a DM, approximating a piston-flow model, as determined by the hydrogeology, well characteristics, and LPM results. A larger component of pre-modern water could have been present in the samples, but was not captured by the estimated mean ages. Helium-4 was the only pre-modern tracer collected, which was difficult to interpret quantitatively, limiting the ability to identify additional pre-modern water. Apparent ${ }^{3} \mathrm{H} / \mathrm{He}$ age was only defined for two samples (RF1 and RFSP) with respective ages of about 25 and 45 years. The apparent ${ }^{3} \mathrm{H} / \mathrm{He}$ age represented the minimum possible age of the young component (modern fraction of a mixture) of the sample as underestimation of ${ }^{3} \mathrm{He}_{\text {trit }}$ resulted in an underestimation of the "true" ${ }^{3} \mathrm{H} / \mathrm{He}$ age. Estimated mean ages were considered more reliable in comparison to ${ }^{3} \mathrm{H} / \mathrm{He}$ ages because there was evidence of error in ${ }^{3} \mathrm{He}_{\text {trit }}$ calculations.

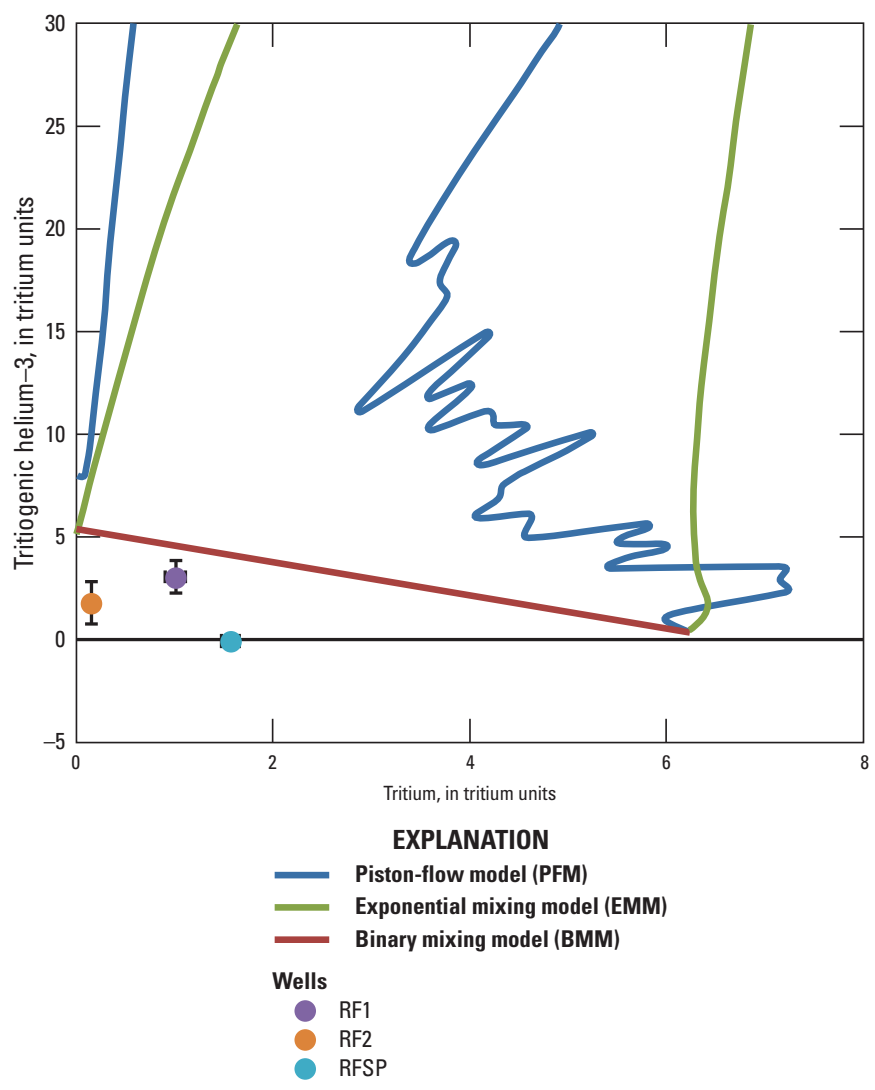

Figure 15. Age-tracer cross-plot of groundwater near Red Fleet Reservoir, Utah.

Table 4. Age-tracer concentrations and estimated mean age and age distribution of groundwater near Red Fleet Reservoir, Utah.

[ID, identification; ${ }^{3} \mathrm{H}$, tritium; TU, tritium units; ${ }^{3} \mathrm{He}_{\text {trit }}$ tritiogenic helium- $3 ;{ }^{3} \mathrm{H}$ age, tritium age; Est, estimated; dist, distribution; RF Res, Red Fleet Reservoir; \pm , plus or minus; - , no data available; EMM, exponential mixing model; RF1 and RF2 are wells; DM, dispersion model; RFSP, Red Fleet State Park]

\begin{tabular}{|c|c|c|c|c|c|c|c|c|}
\hline Station ID & $\begin{array}{c}\text { Sample } \\
\text { name }\end{array}$ & Sample date & $\begin{array}{c}{ }^{3} \mathrm{H} \\
\text { (TU) }\end{array}$ & $\begin{array}{l}{ }^{3} \mathrm{He}_{\text {trit }} \\
\text { (TU) }\end{array}$ & $\begin{array}{c}{ }^{3} \mathrm{H} /{ }^{3} \mathrm{He}_{\text {trit }} \text { age } \\
\text { (years) }\end{array}$ & ${ }^{3} \mathrm{H}$ age & $\begin{array}{c}\text { Est. mean } \\
\text { age } \\
\text { (years) }\end{array}$ & Age dist. \\
\hline 403451109260001 & RF Res & $11 / 14 / 2016$ & $7.9 \pm 0.35$ & - & - & Modern & $25 \pm 5.8$ & EMM \\
\hline 403418109260901 & RF1 & $11 / 13 / 2016$ & $1 \pm 0.1$ & $3.03 \pm 0.8$ & 24.7 & Mixture & $67 \pm 0.2$ & $\mathrm{DM}$ \\
\hline 403508109263901 & RF2 & $11 / 12 / 2016$ & $1.6 \pm 0.09$ & $-0.05 \pm 0.01$ & - & Mixture & $66 \pm 0.1$ & $\mathrm{DM}$ \\
\hline 403506109264201 & RFSP & $10 / 13 / 2016$ & $0.2 \pm 0.03$ & $1.78 \pm 1.02$ & 45 & Pre-modern & $73 \pm 1.8$ & $\mathrm{DM}$ \\
\hline
\end{tabular}




\section{Discussion}

Multiple sources of evidence indicate that groundwater in the Nugget Sandstone aquifer is not significantly recharged by Red Fleet Reservoir. Modeling results with dissolved noble gases in sampled groundwater indicate that the source of groundwater recharge occurs at much lower temperatures more common to high-altitude mountainous regions observed north of the reservoir in the Uinta Mountains. A comparison of major-ion chemistry in sampled Nugget Sandstone aquifer wells near the reservoir and wells several miles from the reservoir indicates similar characteristics in the aquifer regionally that differ from the chemistry observed in Red Fleet Reservoir (table 2). Water levels observed in the Nugget Sandstone near the reservoir also indicate that there is a strong flow gradient from the aquifer to the reservoir with observed water levels in nearby wells ranging from about 35 to $70 \mathrm{ft}$ higher than the maximum reservoir stage (fig. 5). Groundwater chemistry and groundwater-flow gradients indicate that there is limited evidence of groundwater recharging the Nugget Sandstone aquifer immediately surrounding the reservoir, and groundwater discharge from the Nugget Sandstone to the reservoir is the dominant exchange. Conversely, groundwater altitudes in the Frontier Sandstone were 15 to $30 \mathrm{ft}$ lower than the reservoir, indicating that reservoir water is recharging the Frontier Sandstone aquifer.

Trends observed in groundwater discharge volumes calculated in the Red Fleet Reservoir water budget indicate that periods of greatest groundwater discharge occur synonymously during late spring/early summer snowmelt runoff and peaks at similar periods to peakflows observed in the Big Brush Creek discharge record (fig. 7). Results from the seepage study done during baseflow conditions in November 2017 indicate that the reach of Big Brush Creek between the USGS streamgage and the top of Red Fleet Reservoir is a gaining reach. It is likely that some of the groundwater discharge calculated in the Red Fleet Reservoir water budget occurs as seepage to Big Brush Creek before it enters the reservoir.

\section{Summary}

The objectives of this study were to present and interpret (1) groundwater levels, reservoir altitude, meteorological data, and inflows/outflows to and from Red Fleet Reservoir from May 1980 through December 2015 in order to estimate monthly groundwater recharge/discharge to and from surrounding aquifers; (2) aquifer properties for the Nugget Sandstone near Red Fleet Reservoir from a two-well aquifer test; and (3) groundwater and surface-water chemical data to evaluate the origin of water in aquifers adjacent to Red Fleet Reservoir.
From 1980 through 2015, Big Brush Creek discharged water at an average of 29,200 acre-feet per year to Red Fleet Reservoir with a total discharge of about 1,050,000 acre-feet (acre-ft). Maximum and minimum annual discharge from Big Brush Creek to Red Fleet Reservoir from 1980 through 2015 was 49,000 and 12,200 acre-ft, respectively, with the minimum in 2002 and the maximum in 1998. Streamflow discharge from Red Fleet Reservoir is controlled by gates and jet valves at the base of Red Fleet Dam as well as a spillway on the east abutment. Total dam releases from 1980 through 2015 were about 993,000 acre-ft, with annual dam releases during this period ranging from about 15,000 acre-ft in 2013 to about 51,000 acre-ft in 1983. Total evaporation from 1980 through 2015 was about 52,000 acre-ft, with annual evaporation during this period ranging from about 830 acre-ft in 1980 to about 1,770 acre- $\mathrm{ft}$ in 2007 . The average annual pumped volume of water from the Tyzack Pump Station at the base of Red Fleet Dam during years when pumping occurred was about 1,400 acre-ft. The total volume of water pumped from the Tyzack Pump Station from 1980 through 2015 was about 42,500 acre-ft. Total groundwater discharge to Red Fleet Reservoir from 1980 through 2015 was about 40,000 acre-ft.

Based on water-level measurements at six wells and one spring, altitudes of the groundwater in September 2017 near Red Fleet Reservoir in the Nugget and Frontier Sandstones ranged from 5,578 to 5,726 feet (ft). The reservoir altitude during this same period was about 5,590 ft. Groundwaterflow gradients observed in September 2017 in the Nugget Sandstone aquifer indicated that groundwater was discharging to Red Fleet Reservoir from the east and west banks of the reservoir. The water levels in the Frontier Sandstone aquifer observed at well RF2 were about 15-30 ft lower than the stage observed in Red Fleet Reservoir, which indicates a downward groundwater-flow gradient in the Frontier Sandstone west of the reservoir from August 2016 through September 2017.

An aquifer test in the Nugget Sandstone aquifer was completed utilizing the Red Fleet State Park well. This well was pumped at an average rate of 11 gallons per minute from March 9, 2017, to March 17, 2017, as part of an 8-day constant-discharge test. Water-level data were collected at the pumping well and at observation well RF1 for the duration of the test and during a 30-day recovery period so that transmissivity and storage coefficient values of the Nugget Sandstone aquifer could be estimated. Aquifer properties determined by a numerical model for the Nugget Sandstone aquifer near the pumping well yielded a transmissivity of 660 square feet per day with a storage coefficient of 0.13 .

Water samples were collected from four sites near and in Red Fleet Reservoir: two monitoring wells, a culinary well, and surface water from the reservoir. The water samples were analyzed for major ions, nutrients, and selected trace metals to characterize general chemistry and patterns of water quality for a limited area near and in Red Fleet Reservoir. 
Water samples also were analyzed for several environmental tracers including the radioactive isotope of hydrogen (tritium, ${ }^{3} \mathrm{H}$ ), dissolved noble gases, chlorofluorocarbons, and sulfur hexafluoride. The Nugget Sandstone aquifer near Red Fleet Reservoir can be characterized as calcium-magnesiumbicarbonate water with dissolved-solids concentrations that range from 135 to 169 milligrams per liter. The Frontier Sandstone aquifer near Red Fleet Reservoir can be characterized as calcium-magnesium-sulfate water with a dissolved-solids concentration of 2,150 mg/L. Apparent ${ }^{3} \mathrm{H} / \mathrm{He}$ (helium) age was only defined for two samples (wells RF1 and RFSP) with respective ages of about 25 and 45 years. The apparent ${ }^{3} \mathrm{H} / \mathrm{He}$ age represents the minimum possible age of the young component (modern fraction of a mixture) of the sample as underestimation of tritiogenic helium-3 resulted in an underestimation of the "true" ${ }^{3} \mathrm{H} / \mathrm{He}$ age. Recharge temperatures calculated from dissolved noble gases sampled at two wells in the Nugget Sandstone aquifer indicate that high-elevation snowmelt is the primary recharge source. The warmer recharge temperature calculated from noble-gas samples from the Frontier Sandstone aquifer from well RF2 indicates a larger proportion of low-elevation recharge, which is consistent with the idea that the Frontier Sandstone aquifer is recharged by the Red Fleet Reservoir.

\section{References Cited}

Aeschbach-Hertig, W., Peeters, F., Beyerle, U., and Kipfer, R., 2000, Paleotemperature reconstruction from noble gases in ground water taking into account equilibrium with trapped air: Nature, v. 405, no. 6790, p. 1040-1044, https://doi.org/10.1038/35016542.

Clarke, W.B., Jenkins, W.J., and Top, Z., 1976, Determination of tritium by mass spectrometric measurements of ${ }^{3} \mathrm{He}$ : The International Journal of Applied Radiation and Isotopes, v. 27, no. 9, p. 515-522, https://doi.org/10.1016/0020-708X(76)90082-X.

Gardner, P., and Solomon, D.K., 2009, An advanced passive diffusion sampler for the determination of dissolved gas concentrations: Water Resources Research, v. 45, no. 6 , https://doi.org/10.1029/2008WR007399.

Halford, K., Garcia, C.A., Fenelon, J., and Mirus, B.B., 2012, Advanced methods for modeling water-levels and estimating drawdowns with SeriesSEE, an Excel add-in (ver. 1.1, July 2016): U.S. Geological Survey Techniques and Methods 4-F4, 28 p., https://doi.org/10.3133/tm4F4.
Heilweil, V.M., and Susong, D.D., 2007, Assessment of artificial recharge at Sand Hollow Reservoir, Washington County, Utah, updated to conditions through 2006: U.S. Geological Survey Scientific Investigations Report 2007-5023, 14 p., https://doi.org/10.3133/sir20075023.

Heilweil, V.M., Susong, D.D., Gardner, P.M., and Watt, D.E., 2005, Pre- and post-reservoir ground-water conditions and assessment of artificial recharge at Sand Hollow, Washington County, Utah, 1995-2005: U.S. Geological Survey Scientific Investigations Report 2005-5185, 74 p., https://doi.org/10.3133/sir20055185.

Jurgens, B.C., Böhlke, J.K., and Eberts, S.M., 2012, TracerLPM (Version 1): An Excel workbook ${ }^{\circledR}$ for interpreting groundwater age distributions from environmental tracer data: U.S. Geological Survey Techniques and Methods 4-F3, 60 p., accessed March 10, 2017, at https://doi.org/10.3133/tm4F3.

Kipfer, R., Aeschbach-Hertig, W., Peeters, F., and Stute, M., 2002, Noble gases in lakes and ground waters: Reviews in Mineralogy and Geochemistry, v. 47, no. 1, p. 615-700, https://doi.org/10.2138/rmg.2002.47.14.

Lucas, L.L., and Unterweger, M.P., 2000, Comprehensive review and critical evaluation of the half-life of tritium: Journal of Research of the National Institute of Standards and Technology, v. 105, no. 4, p. 541-549, https://doi.org/10.6028/jres.105.043.

Manning, A.H., 2009, Ground-water temperature, noble gas, and carbon isotope data from the Española Basin, New Mexico: U.S. Geological Survey Scientific Investigations Report 2008-5200, 69 p., https://doi.org/10.3133/sir20085200.

Manning, A.H., 2011, Mountain-block recharge, present and past, in the eastern Española Basin, New Mexico, USA: Hydrogeology Journal, v. 19, no. 2, p. 379-397, https://doi.org/10.1007/s10040-010-0696-8.

Manning, A.H., and Solomon, D.K., 2003, Using noble gases to investigate mountain-front recharge: Journal of Hydrology, v. 275, no. 3-4, p. 194-207, https://doi.org/10.1016/S0022-1694(03)00043-X.

Masbruch, M.D., Chapman, D.S., and Solomon, D.K., 2012, Air, ground, and groundwater recharge temperatures in an alpine setting, Brighton Basin, Utah: Water Resources Research, v. 48, no. 10, 12 p., https://doi.org/10.1029/2012WR012100. 
Mazor, E., and Bosch, A., 1992, Helium as a semi-quantitative tool for groundwater dating in the range of 104 to 108 years, in Consultants meeting on isotopes of noble gases as tracers in environmental studies; Panel proceedings series, Vienna, Austria, May 29 to June 2, 1989: Vienna, International Atomic Energy Agency, p. 163-178.

McGuinness, J.L., and Bordne, E.F., 1972, A comparison of lysimeter-derived potential evapotranspiration with computed values - U.S. Department of Agriculture Technical Bulletin 1452: Washington, D.C., Agricultural Research Service, 71 p., http://ageconsearch.umn.edu/ bitstream/171893/2/tb1452.pdf.

Michel, R.L., 1989, Tritium deposition in the continental United States, 1953-83: U.S. Geological Survey Water-Resources Investigations Report 89-4072, 46 p., https://doi.org/10.3133/wri894072.

PRISM Climate Group, Oregon State University, website accessed July 11, 2012, at http://prism.oregonstate.edu.

Rosenberry, D.O., Winter, T.C., Buso, D.C., and Likens, G.E., 2007, Comparison of 15 evaporation methods applied to a small mountain lake in the northeastern USA: Journal of Hydrology, v. 340, no. 3-4, p. 149-166, https://doi.org/10.1016/j.jhydrol.2007.03.018.

Sheldon, A.L., 2002, Diffusion of radiogenic helium in shallow ground water-Implications for crustal degassing: Salt Lake City, Utah, University of Utah, Ph.D. Dissertation, 185 p.

Solomon, D.K., 2000, ${ }^{4} \mathrm{He}$ in groundwater, chap. 14 of Cook, P.G., and Herczeg, A.L., eds., Environmental tracers in subsurface hydrology: Boston, Mass., Kluwer Academic Publishers, p. 425-440, https://doi.org/10.1007/978-1-4615-4557-6_14.
Solomon, D.K., and Cook, P.G., 2000, ${ }^{3} \mathrm{H}$ and ${ }^{3} \mathrm{He}$, chap. 13 of Cook, P.G., and Herczeg, A.L., eds., Environmental tracers in subsurface hydrology: Boston, Mass., Kluwer Academic Publishers, p. 397-424, https://doi.org/10.1007/978-1-4615-4557-6_13.

Sontek, YSI Incorporated, 2009, FlowTracker Handheld ADV Technical Manual Firmware Version 3.7, Software Version 2.30: YSI Incorporated, 116 p.

Stute, M., and Schlosser, P., 2000, Atmospheric noble gases, chap. 11 of Cook, P.G., and Herczeg, A.L., eds., Environmental tracers in subsurface hydrology: Boston, Mass., Kluwer Academic Publishers, p. 349-377, https://doi.org/10.1007/978-1-4615-4557-6_11.

Thompson, F., 1969, Feasibility geology report of the Tyzack Dam and reservoir sites, Jensen Unit, Central Utah Project, Utah: U.S. Bureau of Reclamation Central Utah Project Report G-261.

Turnipseed, D.P., and Sauer, V.B., 2010, Discharge measurements at gaging stations: U.S. Geological Survey Techniques and Methods book 3-A8, 87 p., https://doi.org/10.3133/tm3A8.

U.S. Geological Survey, 2017, The National Map3DEP products and services: The National Map, 3D Elevation Program web page, accessed June 20, 2017, at https://nationalmap.gov/3DEP/3dep_prodserv.html.

Wilde, F.D., and Radtke, D.B., 1998, National field manual for the collection of water-quality data, field measurements: U.S. Geological Survey Techniques of Water-Resources Investigations, book 9, chap. A6, 233 p. 
For more information concerning the research in this report, contact the Director, Utah Water Science Center

U.S. Geological Survey

2329 West Orton Circle

Salt Lake City, Utah 84119-2047

801-908-5000

https://ut.water.usgs.gov

Publishing support provided by the U.S. Geological Survey Science Publishing Network, Sacramento Publishing Service Center 

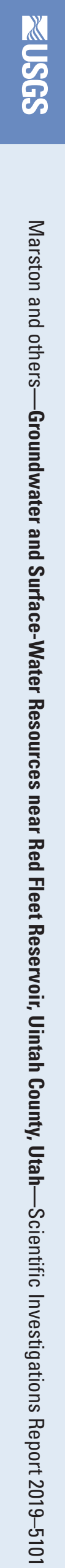